\title{
Radiochromic film for medical radiation dosimetry
}

\author{
Martin J. Butson ${ }^{\mathrm{a}, \mathrm{b}}$, Peter K.N. Yu ${ }^{\mathrm{a}, *}$, Tsang Cheung ${ }^{\mathrm{a}}$, Peter Metcalfe ${ }^{\mathrm{b}}$ \\ ${ }^{a}$ Department of Physics and Materials Science, City University of Hong Kong, Tat Chee Avenue, \\ Kowloon Tong, Hong Kong \\ bIllawarra Cancer Care Centre, P.O. Box 1798, Wollongong 2500, NSW, Australia
}

Accepted 20 June 2003

\begin{abstract}
Photon, electron and proton radiations are used extensively for medical purposes in diagnostic and therapeutic procedures. Dosimetry of these radiation sources can be performed with radiochromic films, devices that have the ability to produce a permanent visible colour change upon irradiation. Within the last 10 years, the use of radiochromic films has expanded rapidly in the medical world due to commercial products becoming more readily available, higher sensitivity films and technology advances in imaging which have allowed scientists to use twodimensional (2D) dosimetry more accurately and inexpensively. Radiochromic film dosimeters are now available in formats, which have accurate dose measurement ranges from less than $1 \mathrm{~Gy}$ up to many kGy. A relatively energy independent dose response combined with automatic development of radiochromic film products has made these detectors most useful in medical radiation dosimetry.

(C) 2003 Elsevier B.V. All rights reserved.
\end{abstract}

Keywords: Radiochromic film; Medical radiation dosimetry; Photon; Electron; Proton

\section{Introduction}

Radiation dosimetry for industrial and medical purposes has steadily evolved over the last few decades with the introduction of various new detectors. Many different detectors have their niche areas of applications depending on the qualities for radiation dosimetry they exhibit. The ideal dosimeter from a physics point of view should be able to measure absorbed dose-energy absorbed per unit mass. Calorimeters, which measure thermal increases in proportion to energy absorbed, are considered primary standard dosimeters. Other dosimeters such as ion chambers are calibrated against calorimeters. This is nearly always performed in national standard laboratories. By comparison of secondary standard dosimeters against the known standards, both absolute and relative dosimetry can then be performed. Absolute dosimetry is a technique that yields information directly about absorbed dose in gray (Gy). It involves many factors, which are used to change the recorded quantity into the absorbed dose. This is normally performed for a standard set. For example, in using a high-energy photon medical linear accelerator, this is often performed with a $10 \mathrm{~cm} \times 10 \mathrm{~cm}$ square irradiation field size, $100 \mathrm{~cm}$ source to surface distance and the detector placed at a depth of $5 \mathrm{~cm}$ in a water phantom. This calibration then relates the machine settings of

\footnotetext{
${ }^{*}$ Corresponding author. Tel.: +852-2788-7831; fax: +852-2788-7830.

E-mail address: peter.yu@ cityu.edu.hk (P.K.N. Yu). 
"monitor units" or "beam on time" to absolute maximum dose per monitor unit in these reference conditions. The term relative dosimetry is given to all subsequent measurements, which are compared to the dose at the absolute calibration point. Examples of this in radiotherapy dosimetry would be the percentage depth dose measurements where the dose at depth is related back to the dose at a specified point or cross profile measurements where the beam dose is measured normal to the beam direction.

Absolute dosimetry can only be performed with a few types of detectors. Important examples are calorimetry, ionometric dosimetry and chemical dosimetry. In these techniques, a constant such as temperature increase yield of a chemical reaction $G$, or the average energy required for the production of an ion pair W/e, is used to convert the measured quantities to the dose.

Most other techniques such as semiconductors, TLDs and film are only suitable for relative dosimetry, i.e. they require a reference measurement to compare against to calculate the results. The ideal dosimeter has a number of features making it useful for dosimetry and some are summarised as follows. Accuracy: The most important feature of any dosimeter is its ability to correctly measure the dose. This is defined as the dosimeter accuracy. The accuracy may be limited by stochastic and systematic errors. Stochastic errors can be reduced by multiple measurements as they are the result of random variations, which may go in all directions and can thus be minimised with a larger data set quantities. Systematic errors can be items such as fogged film, electrometer leakage or repeated shifts in measurement in one direction. Precision: The reproducibility of the results from a measurement technique under similar conditions is defined as its precision. The definition excludes systematic errors and as such does not allow a conclusion to be drawn about the actual correctness of the measured result. It is, however, an important feature for measurements of consistency. Precision is usually defined to a level of 1 or 2 standard deviations of the fluctuations of the measurement around a mean. Detection limit: The detection limit is a guide to the lowest detectable dose with a certain dosimeter type. Readings which include both fluctuations in the natural background and noise within the detector normally determine this level. Measurement range: The measurement range can be defined as the areas from the lowest usable reading to the highest usable reading. Dose response: The reading of the dosimeter should be linearly proportional to the given dose, i.e. the readings for an additional dose should be independent of the dose already registered. Dose rate response: An ideal detector would be independent of dose rate of delivery and this can be particularly important with the use of pulsed high-energy linear accelerators where pulses of high doses of radiation are delivered in short time periods. Energy dependence: For an ideal detector, there should be no difference in the dose response for different radiation qualities. An energy dependence of the dosimeter basically comes down to the fact that different doses can be delivered with the exact same radiation quality but in different materials or tissue types. The requirement of minimal change in dose response with radiation energy usually implies an effective atomic number of the dosimeter being close to that of the material under investigation. This is also an important fact for medical dosimetry where the required absorbed dose, which needs to be measured, is in human tissues of various kinds. Spatial resolution: An ideal dosimeter should be able to determine the dose in an infinitesimally small volume or the point dose. The location and size of this point should also be well defined in the measurement geometry. Practically, all dosimeters have a finite size and the measurement volume is limited by stochastic mechanisms of dose deposition in microscopic dimensions. Finite volumes can then affect dose measurements in regions of high-dose gradients, such as the penumbral regions of high-energy photon beams, which in turn provide inaccurate readings of delivered dose on a microscopic scale. Ease of handling: An ideal detector would be simple to use and physically sturdy enough for clinical and industrial use on a routine basis. There is 
no point having a dosimeter which is accurate but cannot be used in the situations required due to physical limitations of the measurement conditions. For example, a dosimeter which requires the temperature to be $100{ }^{\circ} \mathrm{C}$ for measurement of dose in vivo on a patients skin during radiotherapy treatment would be completely unsuitable.

These above physical characteristics have led scientists to search for a radiation detector with a high spatial resolution, which does not require any special developmental procedure and gives a permanent absolute value of the absolute dose. The dosimetry must also have an acceptable accuracy and precision with a relative ease of handling and data analysis. Some of these features have been achieved with the introduction of radiochromic dosimeters. These dosimeters have a very high spatial resolution and relatively low-energy spectral sensitivity. They are relatively insensitive to visible light and thus offer a unique ease of handling and preparation for a film-type product as they can be handled and prepared under normal room light. Radiochromic dosimeters undergo a colour change directly and do not require chemical processing. The colour change can vary considerably depending on the materials used. However, most radiochromic film dosimeters utilise materials which turn a blue colour when exposed to radiation. The image formation in radiochromic products occurs as a dye forming or a polymerisation process, in which energy is transferred from an energetic photon or particle to the receptive part of the leuko-dye or colourless photo monomer molecule, initiating a colour formation through chemical changes. At present, radiochromic media for dosimetry can be found in various forms including liquid solutions, gels, waveguides and films. Their dosimetric ranges also cover a wide range from doses as low as 0.1 up to $10^{6} \mathrm{~Gy}$. In this review, we aim to specifically cover the characteristics of various film radiochromic dosimeters and their associated characteristics and applications. We will highlight some procedures, which are useful for using these films, and examine various types of densitometers/readers for evaluating dose absorbed.

\section{Radiation interactions with matter (basics for radiation dosimetry)}

Radiation dosimetry is a process whereby a reading is recorded through interactions of the incident radiation with matter causing a measurable change in its properties. This can be a change in the measured charge (ionization chambers), measured light output (TLD) or a visible polymeric chemical reaction (radiochromic film). The process is caused by atomic and nuclear interactions occurring within the atoms. A brief overview of important photon and electron interaction types is given below.

\subsection{Photon interactions in matter}

\subsubsection{Attenuation coefficients}

A characteristic of the interaction of photon radiation with matter is that each individual photon is absorbed or scattered from the incident beam in a single event. The photon number removed $\Delta B$ is proportional to the thickness travelled through $\Delta x$ and the initial photon number $B$, i.e.

$$
\Delta B=-\mu B \Delta x,
$$

where $\mu$ is a constant of proportionality called the attenuation coefficient. In this case, upon integrating, we have

$$
B=B_{0} \mathrm{e}^{-\mu x} \text {. }
$$


The attenuation coefficient is related to the probability of interaction per atom, i.e. the atomic crosssection $\sigma_{\mathrm{a}}$ is given by

$$
\sigma_{\mathrm{a}}=\frac{\mu A}{N_{\mathrm{A}} \rho}
$$

where $A$ is the mass number and $N_{\mathrm{A}}$ the Avogadro's number $\left(6.022 \times 10^{23} \mathrm{~mol}^{-1}\right)$. Table 1 gives a summary of most photon interaction processes with matter [1].

In the energy range of most medical and industrial radiation applications, i.e. nominally less than $20 \mathrm{MeV}$, interactions of photons with matter are mainly due to three processes, these being: (i) photoelectric absorption; (ii) Compton scattering; and (iii) pair production.

As the above-mentioned processes are energy dependent and would all affect the beam, we find that the total attenuation coefficient or cross-section is approximated by the sum of all interactions. For therapeutic photon energies, this consists of absorption and scattering such that

$$
\mu=\tau \text { (photoelectric) }+\sigma \text { (Compton) }+\kappa \text { (pair production }) .
$$

Table 1

\begin{tabular}{|c|c|c|c|c|}
\hline Process & Type of interaction & Other names & $\begin{array}{l}\text { Approximate energy of } \\
\text { maximum importance }\end{array}$ & $\begin{array}{l}\text { Approximate } \\
\text { variation with } Z\end{array}$ \\
\hline Photo electric effect & $\begin{array}{l}\text { With bound atomic } \\
\text { electrons (all energy } \\
\text { given to electron) }\end{array}$ & & $\begin{array}{l}\text { Dominates at low } \\
(1-500 \mathrm{keV}), \text { cross-section } \\
\text { decreases as } E \text { increases }\end{array}$ & $Z^{5}$ \\
\hline \multicolumn{5}{|c|}{ Scattering from electrons } \\
\hline \multirow[t]{2}{*}{ Coherent } & $\begin{array}{l}\text { With bound atomic } \\
\text { electrons }\end{array}$ & $\begin{array}{l}\text { Rayleigh, electron } \\
\text { resonance scattering }\end{array}$ & $\begin{array}{l}<1 \mathrm{MeV} \text { and greatest } \\
\text { at small angles }\end{array}$ & $Z^{2}, Z^{3}$ \\
\hline & With free electrons & Thomson scattering & Independent of energy & $Z$ \\
\hline \multirow[t]{2}{*}{ Incoherent } & $\begin{array}{l}\text { With bound atomic } \\
\text { electrons }\end{array}$ & & $\begin{array}{l}<1 \mathrm{MeV} \text { least at } \\
\text { small angles }\end{array}$ & $Z$ \\
\hline & With free electrons & Compton scattering & $\begin{array}{l}\text { Dominates in region } \\
\text { of } 1 \mathrm{MeV} \text {, decreases } \\
\text { as } E \text { increases }\end{array}$ & $Z$ \\
\hline Nuclear photo effect & $\begin{array}{l}\text { With nucleus as a } \\
\text { whole emitting } \gamma \\
\text { or particle }\end{array}$ & $\begin{array}{l}\text { Particle production, } \\
\text { nuclear } \\
\text { photodisintegration }\end{array}$ & $\begin{array}{l}\text { Above threshold, has } \\
\text { broad maximum range } \\
\text { of } 10-30 \mathrm{MeV}\end{array}$ & \\
\hline \multicolumn{5}{|l|}{ Nuclear scattering } \\
\hline Coherent & $\begin{array}{l}\text { With material as a } \\
\text { whole (dependant on } \\
\text { nuclear energy levels) }\end{array}$ & Nuclear resonance & $\begin{array}{l}\text { Narrow resonance } \\
\text { maximum scattering at } \\
\text { low energies (broad max } \\
\text { in energy range of } \\
10-30 \mathrm{MeV} \text { ) }\end{array}$ & $Z^{2} / A^{2}$ \\
\hline Incoherent & $\begin{array}{l}\text { With individual } \\
\text { nucleons }\end{array}$ & $\begin{array}{l}\text { Nuclear Compton } \\
\text { scattering }\end{array}$ & $\begin{array}{l}\lambda \ll \text { nuclear radius, } \\
\text { i.e. }>100 \mathrm{MeV}\end{array}$ & $Z^{4} / A^{2}$ \\
\hline \multicolumn{5}{|c|}{ Interaction with a coulomb field } \\
\hline Pair production & $\begin{array}{l}\text { In coulomb field } \\
\text { of nucleus }\end{array}$ & Elastic pair production & $\begin{array}{l}\text { Threshold about } 1 \mathrm{MeV} \\
\text { dominates at high } E \text {, i.e. } \\
E>5 \mathrm{MeV} \text { and increases } \\
\text { as } E \text { increases }\end{array}$ & $Z^{2}$ \\
\hline Pair production & $\begin{array}{l}\text { In coulomb field } \\
\text { of electron }\end{array}$ & $\begin{array}{l}\text { Triplet production } \\
\text { inelastic pair prod/n }\end{array}$ & $\begin{array}{l}\text { Threshold at } 2 \mathrm{MeV} \\
\text { increases as } E \text { increases }\end{array}$ & $Z$ \\
\hline Delbruck scattering & $\begin{array}{l}\text { In coulomb field } \\
\text { of nucleus }\end{array}$ & $\begin{array}{l}\text { Nuclear potential } \\
\text { scattering }\end{array}$ & $\begin{array}{l}\text { Real max > imaginary } \\
\text { below } 3 \mathrm{MeV} \text { (both } \\
\text { increase as E increases) }\end{array}$ & $Z^{4}$ \\
\hline
\end{tabular}

Table of radiation interaction processes 


\subsubsection{Photoelectric effect}

With a photoelectric interaction (Fig. 1a), the photon transfers all of its energy to a bound electron and the kinetic energy of the electron is given by Einstein's photoelectric equation:

$$
T_{\max }=h v-\phi,
$$

where $h v$ is the initial photon energy, $\phi$ the electron binding energy (work function) and $T_{\max }=m v^{2} / 2$ the maximum kinetic energy of the ejected electron. The atom is left in an excited state and will emit characteristic radiation and Auger electrons in its transition back to the ground state. The energy deposited in a photoelectric event in tissue can be assumed to be absorbed at the point of photon interaction.

\subsubsection{Compton scattering}

When considering the scattering theory of photons by electrons, it is usually a satisfactory approximation to consider the electrons as free particles [2]. If scattering is considered as in Fig. 1b, relativistic equations derived from the conservation of energy and momentum can be formulated. The change in the wavelength of a photon $(\Delta \lambda)$ is given by

$$
\frac{c}{v^{\prime}}-\frac{c}{v}=\lambda^{\prime}-\lambda=\frac{h}{m c(1-\cos \theta)}
$$

where $\lambda$ is the wavelength of the incident photon, $\lambda^{\prime}$ the wavelength of the scattered photon, $m$ the mass of the electron and $\theta$ the angle of scattering for the photon.

On rearranging, the above equation becomes

$$
h v^{\prime}=\frac{h v}{1+\alpha(1-\cos \theta)},
$$

where $\alpha$ is the ratio of the energy of the photon to the rest energy of the electron.

The kinetic energy $T$ for the recoil electron is given by

$$
T=h v \frac{\alpha(1-\cos \theta)}{1+\alpha(1-\cos \theta)}
$$

and the scattering angle of the electron is given by

$$
\cot \phi=(1+\alpha) \tan \frac{1}{2} \theta .
$$

Klein and Nishina [3] carried out a quantum mechanical treatment to obtain the Compton scattering cross-section using the Dirac equation for the electron. This reduces to the classical Thomson scattering equation, for low-energy photons when $\alpha \ll 1$.

\subsubsection{Pair production}

The production of a positive and negative electron pair (pair production) is a process that can take place in the vicinity of the field of an atom or an electron as shown in Fig. 1c. Absorption of photons through the mechanism of pair production can occur when the energy of an incident photon is greater than twice the rest mass of an electron, i.e. $2 \times 0.511 \mathrm{MeV}=1.022 \mathrm{MeV}$ [4]. During pair production interactions, a photon has its energy converted to an electron-positron pair. The positron so produced interacts with matter by ionising and exciting atoms through the same processes as 
(a)

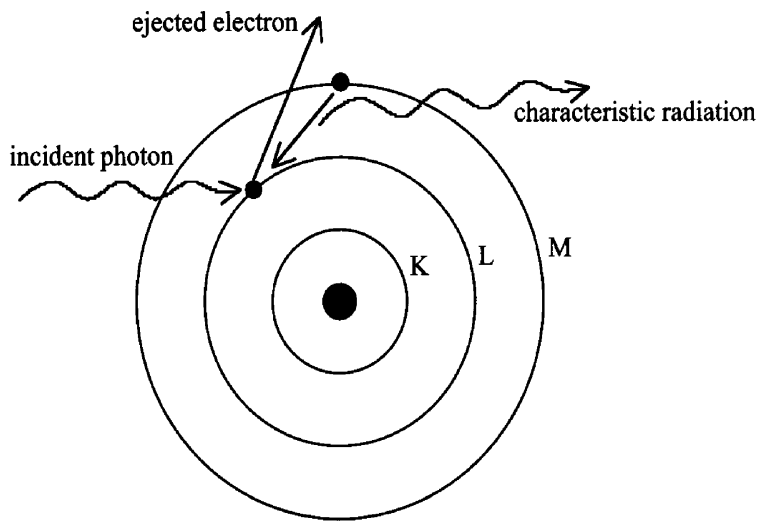

(b)
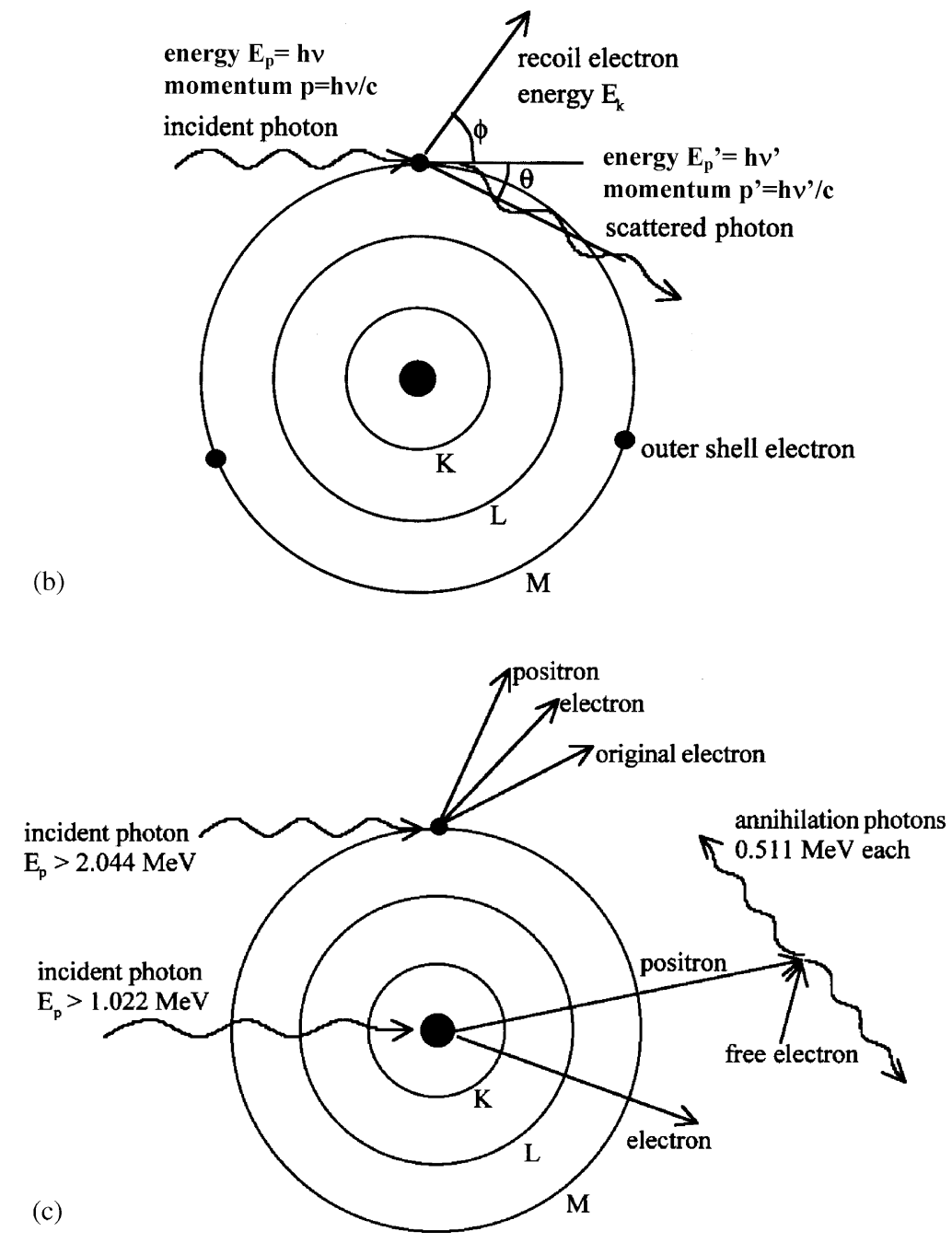

Fig. 1. (a) The photoelectric interaction process. (b) The Compton interaction process with an M-shell electron. The photon is scattered and a recoil electron is produced. (c) The pair production interaction process. 
electrons, thus losing energy and being brought to rest. At this point, the positron combines with an electron in an annihilation process producing two $0.511 \mathrm{MeV}$ photons.

\subsubsection{Electron disequilibrium}

Mono-energetic electrons (produced by X-ray interactions) can have different ranges and angles of interaction on a microscopic scale. This phenomenon can be visualised by assuming monoenergetic electrons and looking at their longitudinal range component. There is a point at which the intensity of electron dose deposition reaches a maximum. If there were no attenuation then longitudinal equilibrium would exist from this depth onwards $\left(d_{\max }\right)$. Before this region, there is a difference between entering and exiting electron intensity. This is known as longitudinal electronic disequilibrium. In reality, there is attenuation of the fluence beyond $d_{\max }$ and hence slightly fewer electrons are being generated than deposited at points past $d_{\max }$. Hence, at depths beyond $d_{\max }$ in the dose fall off region the beam is said to be in a state of transient longitudinal electron equilibrium.

\subsubsection{Importance of each interaction process}

It has been shown in the previous section that the probability of each interaction occurring varies with the incident photon energy $h v$, and the atomic number $Z$ of the absorber. Fig. 2 shows the relative proportion of the three main interaction processes taking place in water plotted against energy. Compton interactions are the main process at therapeutic energies. Results are plotted using relative percentage data for interactions from Johns and Cunningham [4].

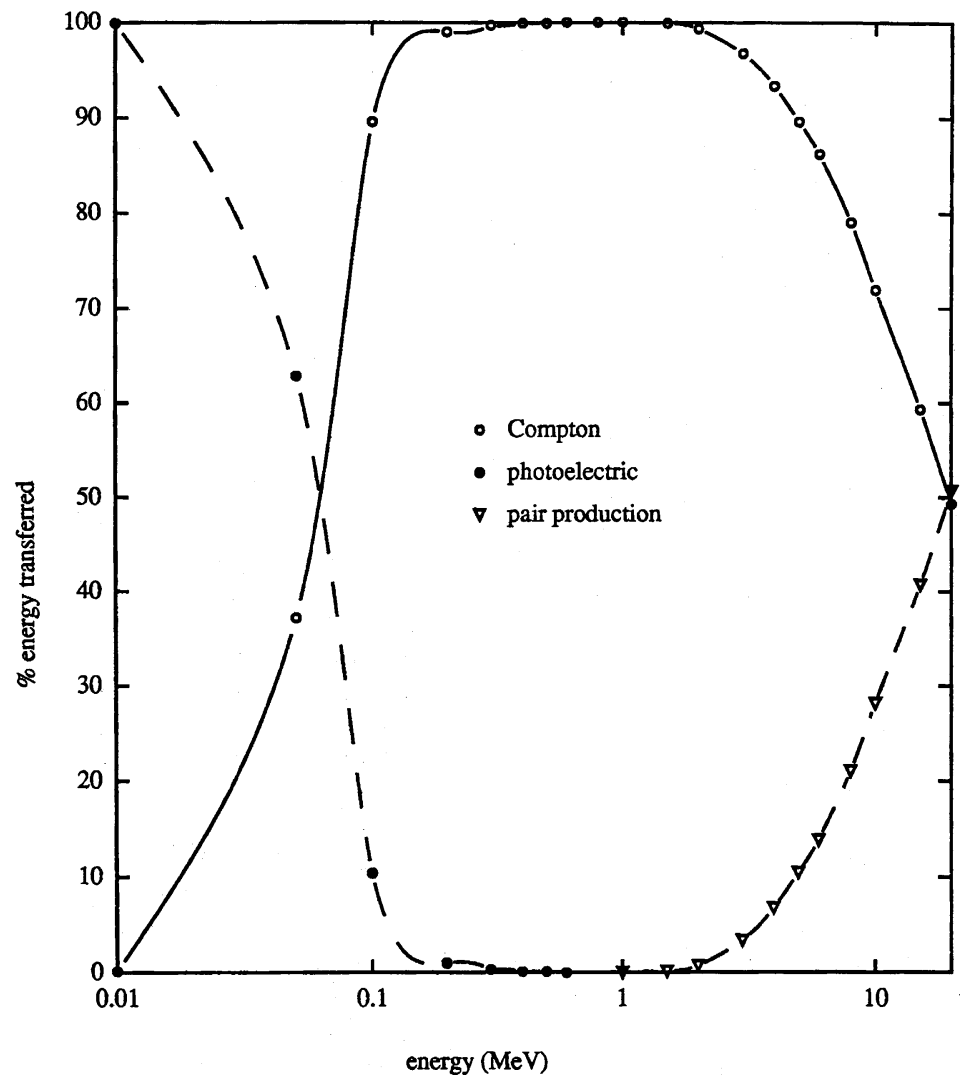

Fig. 2. Percentage contributions of photoelectric, Compton and pair production interactions for photons at various energies. At medical clinical energies, Compton interactions are the main process. 


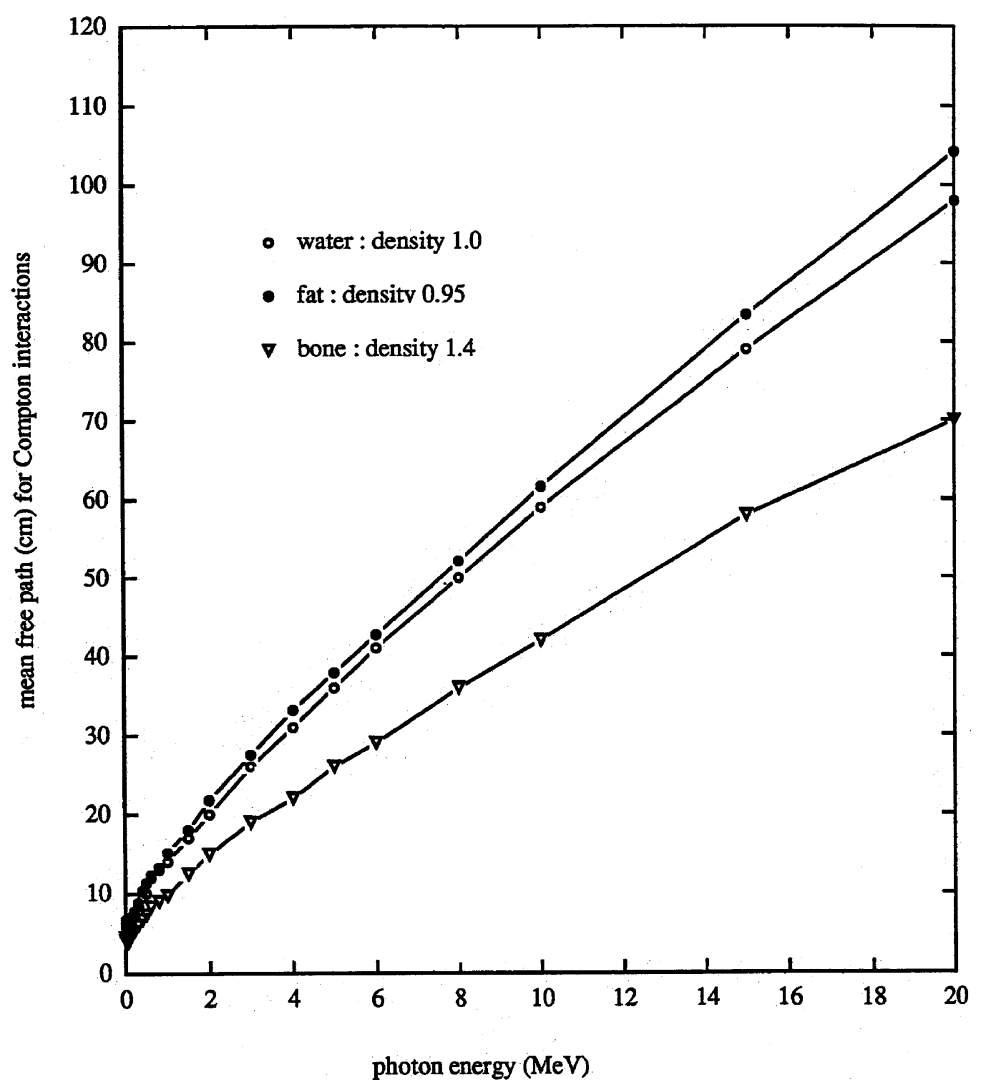

Fig. 3. Mean free path of photons in water, fat and bone. The average energy for a $6 \mathrm{MV}$ clinical photon spectrum is approximately $2 \mathrm{MeV}$, for which the mean free path in water is approximately $20 \mathrm{~cm}$.

\subsubsection{Mean free path}

The mean free path of photons in matter is given by the reciprocal of the linear attenuation coefficient. It represents the average distance a photon will travel before interacting. There will be a statistical variance around the mean value which normally follows a Gaussian shape. Fig. 3 shows the mean free paths for photons in water, fat and bone. On average, relatively large distances are travelled before interactions take place. For photons in water at $6 \mathrm{MeV}$, the mean free path is approximately $40 \mathrm{~cm}$.

At $2 \mathrm{MeV}$ (approximately the mean energy for a $6 \mathrm{MV}$ spectrum), the mean free path is approximately $20 \mathrm{~cm}$ in water, and about $170 \mathrm{~m}$ in air.

\subsection{Electron interactions}

When an X-ray photon interacts with a medium, any one of the three major photon interaction processes previously outlined may occur. These interactions result in charged particles, electrons or positrons, "ranging" from the original site of photon interaction. These charged particles will be referred to as electrons in this section. The term "ranging" is used to describe the process whereby electrons travel from the sites of photon-electron interaction and undergo multiple electron-electron and electron-nucleus interactions prior to coming to rest, having deposited energy along their tracks. The interactions of electrons with electrons lead to ionization and excitation processes responsible for biological damages to the cells. 


\subsubsection{Collisional energy loss}

Collisions, which cause electron energy loss, are of major interest as these events give rise to deposition of energy. Collisional energy loss occurs when a ranging electron interacts with atomic electrons and this results in either excitation or ionization of the atom. In general, this process is closely modelled by a free electron collision because the outer shell electrons are loosely bound.

Collisional energy losses where the electron loses a small amount of energy are very frequent. The rate of energy loss by this mechanism depends on the electron energy and the ionization energy of the atoms in the medium. Large energy losses occur less frequently where a significant proportion of the energy of ranging electrons is transferred to an orbital electron, which is called a knock-on collision, and the ejected electron is referred to as a $\delta$-ray.

\subsubsection{Radiative energy loss (Bremsstrahlung production)}

When an electron passes close to the nucleus of an atom, it experiences an electromagnetic force and results in an energy loss. Bremsstrahlung radiation is generated in the form of photon emission when an electron interacts with the coulomb field of the nucleus. The probability of such an interaction increases as the distance of the electron's approach to the atom decreases. The maximum energy of the Bremsstrahlung photon cannot be larger than the incident electron energy and a spectrum of photon energies below this value is produced. Since the energy lost is converted to a Bremsstrahlung photon, this process is referred to as a radiative energy loss.

The energy loss due to production of photons per path length $\mathrm{d} z$ from electrons of energy $E=h v_{\max }$, where $v$ is the Bremsstrahlung frequency, is given approximately by the following relationship [5]

$$
\left(\frac{\mathrm{d} E}{\mathrm{~d} z}\right)_{\mathrm{rad}}=4 Z^{2} \frac{N}{137} r_{0}^{2} E \frac{183}{Z^{1 / 3}},
$$

where $Z$ is the atomic number, $N$ the number of nuclei per unit volume and $r_{0}$ the classical radius of the electron.

\subsubsection{Electron stopping powers}

The energy transferred from the electron to the medium by collisional or radiative processes is quantified by the use of stopping powers. The rate of energy loss per unit path length $\mathrm{d} E / \mathrm{d} z$ is termed the total stopping power and has components from radiative and collision losses. To make these quantities mass independent, the mass stopping powers are also defined. The total mass stopping power is equal to the sum of the collisional stopping power $\left(S_{\mathrm{col}} / \rho\right)$ and radiative mass stopping power $\left(S_{\mathrm{rad}} / \rho\right)\left(\mathrm{MeV} \mathrm{cm}^{2} \mathrm{~g}^{-1}\right)[5]$

$$
\frac{S_{\mathrm{tot}}}{\rho}=\left(\frac{S_{\mathrm{rad}}}{\rho}\right)+\left(\frac{S_{\mathrm{col}}}{\rho}\right)
$$

Tables of stopping powers are available [6]. The collisional stopping power is usually represented by a special case called the restricted stopping power $L$. This is because if enough energy is imparted in the collision to the atomic electron, the latter can have sufficient energy to itself cause ionization. The actual energy deposited at a point is therefore defined as the energy transferred by secondary electrons in collisional energy losses less than a specific value defined as $\Delta$. The linear restricted stopping power, also known as the linear energy transfer $L_{\Delta}$, is therefore the energy loss per unit path length in which the energy loss per collision is less than $\Delta$. Note that if there is no restriction placed on the energy loss, we have $L_{\Delta}=L_{\infty}=S_{\text {coll }}$. 
The restricted mass stopping power is therefore defined as

$$
\left(\frac{L}{\rho}\right)_{\Delta}=\left(\frac{S_{\mathrm{col}}}{\rho}\right) \quad(\text { energy loss }<\Delta),
$$

where the parameter $\Delta$ is typically set at $100 \mathrm{keV}$ as the range of electrons in water at such a low energy is less than $1 \mathrm{~mm}$.

\subsubsection{Electron scattering powers}

Electrons are continuously deflected by electromagnetic interactions with nuclei. These are predominantly frequent, small-angle deflections, but less frequent large-angle scattering also occurs.

The net angular deflection, which an electron undergoes in traversing a certain thickness of material due to small-angle scattering, is a statistical quantity which can be calculated using multiple coulomb scattering theory. In this theory, the mean square angular deflection per unit path length is called the scattering power $\left(\mathrm{d} \theta^{2} / \mathrm{d} z\right)$. The scattering power decreases with the electron energy and increases with the atomic number of the medium. The multiple coulomb scattering theory has been used successfully to model the scattering of electrons in electron beam treatment planning algorithms.

The approach put forward by Fermi [5] to model the predominant multiple small-angle scattering, which characterises the electron beam, was to formulate scatter distribution functions for lateral displacement and angular deflection. Fermi's original method assumed the electron energy remained constant with depth. Eyges [7] provided a modified solution to account for beam energy degradation of about $2 \mathrm{MeV} \mathrm{cm}^{-1}$, where:

$$
E_{z}=E_{0}\left(1-\frac{z}{R_{\mathrm{p}}}\right)
$$

$E_{z}$ is the energy at the depth $z, E_{0}$ the energy incident at the surface and $R_{\mathrm{p}}$ the practical range of electrons. Although this is an approximation, the theory works well and successful computerised electron pencil beam planning models, such as those employed by Hogstrom [8], are based on the Fermi-Eyges scattering theory. Complex beam situations can be reasonably accurately modelled using the pencil beam approach [9-11].

There are some situations where the failure of the method to model large-angle scattering causes errors. These problems are addressed by Brahme [12] and Lax [13]. These groups are producing slightly non-Gaussian shaped pencil beams by using Monte Carlo techniques. For a more complete analysis of electron scattering, the reader can refer to the SLAC-265 report [14].

\subsection{Fluence, KERMA and absorbed dose}

Photon fluence, KERMA and absorbed dose are defined by the International Commission of Radiation Units, in the reports ICRU\#19 [15] and ICRU\#24 [16], as follows.

\subsubsection{Fluence}

Photon fluence, $\mathrm{d} N / \mathrm{d} a$, is the number of photons which cross a unit cross-sectional area. Therefore, the photon fluence $\Phi$ and the energy fluence $\Psi$ are defined, respectively, as

$$
\Phi=\frac{\mathrm{d} N}{\mathrm{~d} a}
$$


and

$$
\Psi=\frac{\mathrm{d} N}{\mathrm{~d} a} h v=\Phi h v .
$$

The planar photon fluence refers to the number of photons which cross a plane per unit area. Hence, the planar fluence is dependent on the incident beam angle whereas the photon fluence is not.

\subsubsection{KERMA and TERMA}

The Kinetic Energy Released in a medium per unit Mass or KERMA, where $K$, represents the average energy transferred from the photon to the charged particle $\mathrm{d} E_{\text {tr }}$ per unit mass $\mathrm{d} m$ at the site of interaction. Therefore,

$$
K=\frac{\mathrm{d} \bar{E}_{\mathrm{tr}}}{\mathrm{d} m}
$$

As KERMA is the kinetic energy released, it is equal to the energy fluence $\Psi$ multiplied by the coefficient of energy transfer $\mu_{\mathrm{tr}} / \rho$. Therefore,

$$
K=\Psi\left(\frac{\mu_{\mathrm{tr}}}{\rho}\right)
$$

and so:

$$
K=\Phi(h v)\left(\frac{\mu_{\mathrm{tr}}}{\rho}\right)
$$

As pointed out by Attix [17], the KERMA can be partitioned into two components: the energy transferred to charged particles which results in energy deposition by collisional energy losses, $K_{\text {col }}$, and the energy transferred which results in radiative energy losses, $K_{\text {rad }}$. Therefore,

$$
K=K_{\mathrm{col}}+K_{\mathrm{rad}}=\left(\frac{\mu_{\mathrm{ab}}}{\mu_{\mathrm{tr}}}\right) K+\left(1-\frac{\mu_{\mathrm{ab}}}{\mu_{\mathrm{tr}}}\right) K .
$$

Since collisional KERMA is the amount of energy per unit mass transferred to electrons by photons (with the exception of radiative losses), this is a measure of the amount of energy dissipated locally by ionization events, which can be described by

$$
K_{\mathrm{col}}=E_{v} \Phi_{v}\left(\frac{\mu_{\mathrm{en}}}{\rho}\right)
$$

where $E_{v}$ is the average energy of the photon $(\mathrm{J}), \Phi_{v}$ the photon fluence at the point $\left(\mathrm{m}^{-2}\right)$ and $\mu_{\mathrm{en}} / \rho$ the mass energy absorption coefficient averaged over the energy fluence spectrum of the photons. Furthermore,

$$
K_{\mathrm{col}}=K(1-g),
$$

where $g$ is the average fraction of energy lost via radiative processes.

The KERMA only includes the kinetic energy transferred to the charged particle whereas some energy is usually retained by the photon as it is scattered away from the interaction site (as in 
Compton interactions). The Total Energy Released in a medium per unit Mass, which includes both these quantities, is defined as the TERMA, where

$$
T=\Psi\left(\frac{\mu}{\rho}\right)
$$

\subsubsection{Absorbed dose and exposure}

Although KERMA describes that part of the incident energy which is converted to kinetic energy of electrons, it does not indicate how much energy is actually retained by the medium as heat. This is because electrons radiate away some of their energies as Bremsstrahlung. Therefore, the KERMA released at a point in the medium causes energy to be deposited throughout the path of liberated electrons travelling down stream (or in rare cases upstream) of the interaction site.

The absorbed dose at a point is the energy deposited by electrons per unit mass at that point from collisional energy losses. The magnitude of the dose is therefore almost the same as $K_{\text {col }}$.

Provided charged particle equilibrium exists (as described in Section 2.1), then for a particular medium (med):

$$
D_{\text {med }} \stackrel{\text { PPE }}{=}\left(K_{\text {col }}\right)_{\text {med }} \text {. }
$$

If the medium is a gas, an electron loses energy by ionising the gas. The quantity $W$ (eV per ion pair) is the mean energy expended in the gas per ion pair produced. This quantity is constant in dry air for electron energies above a few $\mathrm{keV}$. By dividing $W$ by $e$ (the charge of the electron), we have a term describing the energy $(\mathrm{J})$ deposited in air per unit charge $(\mathrm{C})$ released:

$$
\left(\frac{W}{e}\right)_{\text {air }}=33.97 \mathrm{~J} \mathrm{C}^{-1} \text {. }
$$

Note also that $(e / W)$ gives the coulombs of charge released per Joule of energy.

The exposure $X$ is the absolute value of charge $\mathrm{d} Q$ produced by ions (of one sign) in dry air when all the electrons liberated in air of mass $\mathrm{d} m$ are completely stopped, i.e.

$$
X=\frac{\mathrm{d} Q}{\mathrm{~d} m} .
$$

Note that ionization is the "ionization equivalent" of the collisional KERMA in air for photons, i.e.

$$
D_{\text {air }} \stackrel{\mathrm{CPE}}{=}\left(K_{\mathrm{col}}\right)_{\mathrm{air}}=X\left(\frac{W}{e}\right)_{\text {air }} .
$$

The energy absorbed by a medium per unit mass, or the absorbed dose $D$, represents the energy deposited by charged particles in the medium $\mathrm{d} E_{\mathrm{ab}}$ per unit mass $\mathrm{d} m$, where

$$
D \approx \frac{E_{\mathrm{ab}}}{\mathrm{d} m} .
$$

However, to be more rigorous, the energy absorbed by a medium per unit mass, or the absorbed dose $D$, represents the average energy $\mathrm{d} \bar{\varepsilon}$ deposited by charged particles in the medium per unit mass $\mathrm{d} m$, where $\mathrm{d} m$ is infinitesimally small but is large enough to prevent stochastic variations [15], such that

$$
D=\frac{\mathrm{d} \bar{\varepsilon}}{\mathrm{d} m}
$$


The mean energy $\mathrm{d} \bar{\varepsilon}$ is an average of the energy $\mathrm{d} \varepsilon$ deposited in $\mathrm{d} m$ over successive measurements [18]. While $\mathrm{d} \varepsilon$ is a stochastic quantity since it varies discontinuously over time, the mean $\mathrm{d} \bar{\varepsilon}$ is a nonstochastic quantity which is a continuous function of time. Another way of expressing $D$ is

$$
D=\lim _{v \rightarrow 0} \frac{\mathrm{d} \bar{\varepsilon}}{\rho \mathrm{d} v}
$$

where $v$ is a volume whose limit is approaching zero.

\subsection{Medical radiation summary}

During a photoelectric interaction, the photon gives up all its energy to an electron, which escapes from the atom with the kinetic energy being equal to that of the photon minus the shell binding energy. If the atom is left in an excited state, an outer shell electron may fill the inner shell vacancy and the energy difference is given off as a characteristic radiation. The photoelectric component of the mass attenuation coefficient varies approximately as $Z^{3}$ ( $Z$ is the atomic number of the material) and is reduced approximately with $1 / E^{3}$.

Compton scattering is also known as incoherent scattering in which a photon collides with an electron, transferring momentum to a recoil electron. The photon is scattered with an energy smaller than (or a wavelength longer than) that of the incident photon. The electron escapes and the shell vacancy may be filled by an Auger electron or an outer shell electron, which in turn gives off characteristic radiation. Since momentum is conserved, the recoil angle of the electron is uniquely related to the scattering angle of the photon. This relation is characterised by the Klein-Nishina formula, which can be used to calculate the differential cross-section per unit solid angle, and hence the Compton component of the mass attenuation coefficient. This component is approximately proportional to $Z$. The Compton component increases with energy up to about $0.1 \mathrm{MeV}$ and remains the dominant component until about $20 \mathrm{MeV}$.

Pair production may occur only if the incident photon energy is greater than or equal to two electron mass units $(1.022 \mathrm{MeV})$. In this process, the photon interacts with the field of the nucleus to produce an electron-positron pair. Any energy, which the photon has in addition to the $1.022 \mathrm{MeV}$, is shared between the positron and the electron. The positron deposits energy in a manner similar to the electron (e.g. ionization events), but when at rest it quickly annihilates with another electron to produce two photons of energy $0.511 \mathrm{MeV}$, which travel in opposite directions to each other. The pair production mass attenuation coefficient component increases with $Z$ and increases rapidly with increasing photon energy above $1.022 \mathrm{MeV}$. It is the dominant process above about $20 \mathrm{MeV}$ (the exact energy depending on the $Z$ of the material).

Unlike photons, electrons are capable of directly depositing energy in a medium. Whether incident on the medium or liberated within it by photon interactions, the electrons set in motion interact in a number of ways. The measure of the rate of energy loss is known as the mass stopping power $\left(S_{\text {tot }} / \rho\right)$. The concept of mass angular scattering power is also used to describe the way electrons interact, which is equal to the mean square angular deflection per unit mass per unit distance $\left(\mathrm{d} \theta^{2} /(\rho \mathrm{d} z)\right)$.

During collisional energy loss, the ranging electron causes excitation or ionization of a bound electron. Occasionally, the electron has sufficient energy (approximately $100 \mathrm{eV}$ ) to range away as a distinct ionising particle, which is called a $\delta$-ray. In general, the incident electron continues with slightly reduced energy, and may undergo many thousands of ionisations before coming to rest. 
Radiative energy loss occurs when the electron is scattered by the field of the nucleus and a Bremsstrahlung photon is given off. At low energies, the direction of maximum intensity of radiation is given off at right angles to the electron beam (e.g. diagnostic X-ray reflective target), but at megavoltage energies, the Bremsstrahlung is emitted as a narrow bullet shaped lobe (e.g. linear accelerator transmission target). This lobe is then flattened using a metal flattening filter (usually stainless steel).

Due to electron ranging, energy deposition does not occur at the position of photon-electron interaction. Electrons can range several centimeter from the photon-electron interaction site. The electrons have a significant longitudinal and more importantly lateral range [19], which in various situations can lead to lateral electronic disequilibrium.

\section{Medical film dosimetry}

\subsection{Optical density to dose relationship}

When radiochromic film is exposed to ionising radiation, colouration occurs. This colouration is due to an attenuation of some of the visible light coming through the developed film, resulting in a 'greying' of its appearance. The reduction in light passing through the film is a measure of its 'blackness' or 'optical density' (OD). A pivotal assumption in film dosimetry is that the dose to the film is reflected in the resulting optical density of that film. This relationship can be expressed as follows:

$$
\text { optical density }=\log _{10}\left[\frac{I_{0}}{I}\right]
$$

where $I_{0}$ is the light intensity with no film present and $I$ is the light intensity after passing through the film. Note that since $I_{0} / I$ has an exponential relationship to the dose, the optical density is appropriately linear with dose. The acceptance of this relationship has led to the wide use of the film as a dosimeter [20-23]. The advantages offered by the film as compared to other dosimeters include the mapping ability whereby an area of dose can be analysed as compared to a point measurement in most other types of detectors. In other words, a two-dimensional (2D) optical density fluence map is produced. With appropriate corrections, this can be converted to a 2D dose map, which can also be viewed conveniently. In most traditional uses of film dosimetry, only relative dosimetry is performed. It is assumed that if there is any inaccuracy, it is constant throughout all of the readings, i.e. a systematic error. The film dosimetry results are usually verified by a second dosimeter, e.g. point dose measurements using an ionisation chamber. This approach has been able to provide useful dosimetric information.

The information from film dosimetry can be utilised in two ways, either qualitatively or quantitatively. Without a doubt, the film has no equal for communicating qualitative information about the dose distribution. However, accurate quantitative film dosimetry presents a few technical challenges.

\subsection{Potential variables of film dosimetry}

The principal concern with using film as a dosimeter is the fragility of the relationship between dose and optical density. This relationship can also be expressed as the sensitivity of the film to dose. 
It has been suggested that the film sensitivity is affected by the photon beam energy, emulsion differences between film batches, film plane orientation, experimental design, post-irradiation conditions and types of densitometers used or more broadly the analysis tool. A film may also be sandwiched between the solid water slabs and exposed parallel to the beam direction. Perpendicular exposure of a film seems to generate fewer uncertainties in terms of air gaps in solid water and the energy response can be better characterised by calibrating a batch of film at the same depth. Note that the quantitative analysis of parallel-exposed film presents a challenge for accurate dose results as the calibration curves are depth dependent and the air gap and film perturbation effects are greater for the parallel exposure. Several authors have investigated these variables and their effects on the relationship between the dose and the optical density. They are in agreement on some variables while they contradict each other on others, and some variables have not been addressed in the literature at present.

\section{Radiochromic film}

\subsection{Chemical, physical and radiation process}

Radiochromic reactions by definition are a direct colouration of a media by the absorption of radiation, which does not require any latent thermal, optical or chemical development or amplification [24-27]. Radiochromic reactions were first observed and recorded by Niepce in 1826. This observation involved an unsaturated hydrocarbon polymeric mixture based on bitumen that cross-linked upon irradiation, which left a light scattering pattern (quoted from [28]). Radiochromic organic image-forming systems can involve cis-trans isomeric dissociations or conversions, which can result in ketonic, anilic and enolic bonds. These tautomerisations can lead to double-bonded colourations of anils organic acids, stilbenes and other polycyclic compounds [29,30]. Other radiochromic effects include radiation-induced vesicular films giving light scattering properties [31] or radiation-induced hardness of polymeric microcapsules, containing diffuseable dyes which are released mechanically [32,33]. Another image-forming technique is the use of organic-free radical imaging medium which combines photo-polymerisation with leuco dyes that produce colour upon irradiation. This results in the pairing of free radicals to form radiation-induced cross-linked carbon chains which result in covalently bonded growing chains.

In more recent years, the use of radiochromic materials in the form of films have become widely used as dosimeters for industrial and medical applications. Colourless transparent radiochromic films which give a permanent colour change have been used in high-dose applications for the past 35 years $[24,34,35]$. These dosimeters are mainly hydrophobic-substituted triphenylmethane leucocyanides that undergo a heterolytic bond scission of the nitrile group, which forms a highly coloured dye salt in solid polymeric solution when irradiated. These molecules require a host material for film development, which normally consists of a strylene-, vinyl- or nylon-based polymer. These types of film do however require large doses to deliver a distinguishable change in colour, typically in the order of $10^{4}-10^{6}$ Gy [24]. A more recent form of radiochromic film has been based on polydiactylene and has found extensive use in medical applications where low doses are required to be measured [36-38]. These types of films are supplied in various forms to match the specific need in medical dosimetry with dosimetric ranges from 2500 down to $1 \mathrm{~Gy}$, if required. Specific film types, which are commonly used for industrial and medical purposes, will be investigated and treated separately within this review and the physical and radiation properties reported.

As many materials possess radiochromic properties, a multitude of variability arises in the physical characteristic of these products. In medical dosimetry, GAFCHROMIC ${ }^{\circledR}$ dosimetry film is 


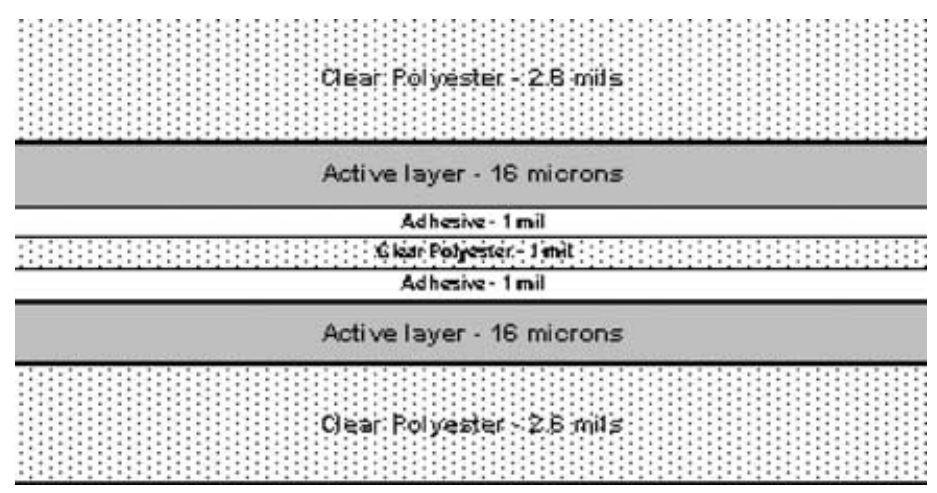

Fig. 4. Physical structure of ISP GAFCHROMIC ${ }^{\circledR}$ MD-55-2 radiochromic film, which is a typical radiochromic film product used for medical dosimetry. (Courtesy of David Lewis, ISP Technology).

one of the major products available from ISP Technology and is arguably the most widely used commercial product. To simplify our review paper, we have chosen their product GAFCHROMIC ${ }^{\mathbb{R}}$ MD-55 to exhibit the general radiation and material properties of the radiochromic film. The physical structure of the GAFCHROMIC ${ }^{\mathbb{R}}$ MD-55-2 radiochromic film is shown in Fig. 4. It is noted that many variations occur for other commercial and non-commercial products.

Radiochromic dosimetry film is designed for the measurement of absorbed dose of high-energy photons, electrons and protons. The kinetics of the radiochromic reaction of one of the GAFCHROMIC $^{\circledR}$ product range (MD-55-1) has been evaluated by McLaughlin et al. [39-41] using flash photolysis and pulse radiolysis. Their investigations showed that the radiochromic reaction was indeed a solid-state polymerisation in which the film undergoes progressive 1,4-trans additions, producing a polyconjugated polymer chain which exhibits the blue colouration from radiation exposure. A first-order rate constant, of the order $10^{3} \mathrm{~s}^{-1}$, was found with an activation energy of approximately $50 \mathrm{~kJ} \mathrm{~mol}^{-1}$ for the pulse electron-induced propagation of the polymerization.

The active radiochromic film layer is approximately $16 \mu \mathrm{m}$ thick and is coated onto a transparent $67 \mu \mathrm{m}$ thick polyester sheet. For construction of the MD-55-2 film, two pieces of this film construction are laminated together with a dual-sided bonding tape. The tape is constructed from a $25 \mu \mathrm{m}$ thick adhesive layer on both sides of a $25 \mu \mathrm{m}$ clear polyester base. This construction provides a total active layer of approximately $32 \mu \mathrm{m}$. The active layer thickness will vary slightly from batch to batch, as specified by the manufacturer, in order to provide a more stable sensitometric response due to the slight variations in the active layer sensitivity per batch. The manufacturer will normally supply details concerning the sensitivity of the required batch upon request.

\subsubsection{Optical density properties of typical radiochromic film (MD-55-2 GAFCHROMIC ${ }^{\circledR}$ film)}

Fig. 5 shows the dye light absorption spectra for the MD-55-2 radiochromic film, which has been irradiated to various doses ranging from 0 up to $60 \mathrm{~Gy}$.

These results are for a film from the batch no. 37350. The film produces a blue colour upon irradiation, which is evident by the large spectrum absorption in the red region as shown in the figures. The radiochromic layer in a GAFCHROMIC ${ }^{\circledR}$ MD-55-2 film contains micro-crystals of a monomer. This layer undergoes partial polymerization by the ionising radiation and becomes darker with more absorbed dose. Two main absorption peaks occur in the visible waveband, these being at 617 and $675 \mathrm{~nm}$. A small shift in the position of these peaks occurs with the delivered absorbed dose (main peak at $675 \mathrm{~nm}$ for $0 \mathrm{~Gy}$ with a shift to $676 \mathrm{~nm}$ at $6 \mathrm{~Gy}$ [42] and the secondary peak shifts 
Absolute Optical Density for irradiated MD-55-2 Gafchromic film

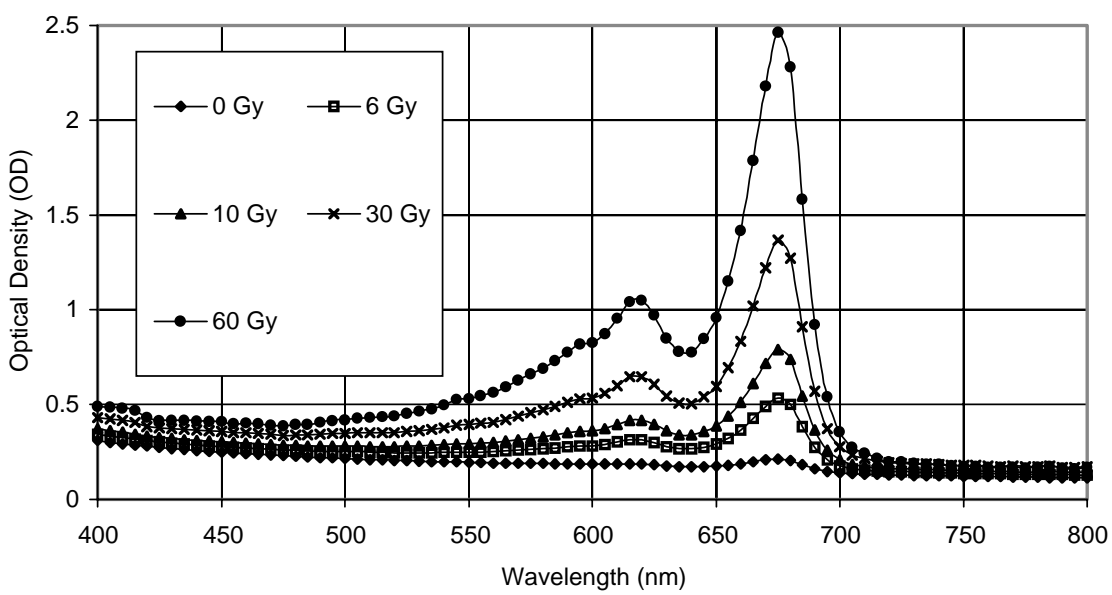

Fig. 5. Dye light absorption characteristics of the MD-55-2 radiochromic film. The absorption spectrum is shown in the visible region with main absorption peaks located in the red region; hence a blue colour upon inspection of the film induced from radiation absorption.

from $617 \mathrm{~nm}$ at 0 Gy to $618 \mathrm{~nm}$ at $6 \mathrm{~Gy}$ ). Also shown in Fig. 5 is the "background" optical density for the unirradiated film at all wavelengths, which comes from a small proportion of coloured polymer as well as the base material. Some of this background OD is due to absorption and some due to reflection [42]. When the background OD is removed from the curve, a net optical density measurement is produced and shown in Fig. 6 for the MD-55-2 film. This is a direct subtraction of the optical density results for a film in its unirradiated state from those in its radiation-exposed state.

Fig. 7 shows the dose response of the GAFCHROMIC ${ }^{\mathbb{R}}$ MD-55-2 film at specific wavelengths of determination. The results show a large variation in sensitivities achievable through analyses at different wavelengths of the visible spectra. The maximum sensitivity is produced at the maximum

Net Optical Density for irradiated MD-55-2 Gafchromic film

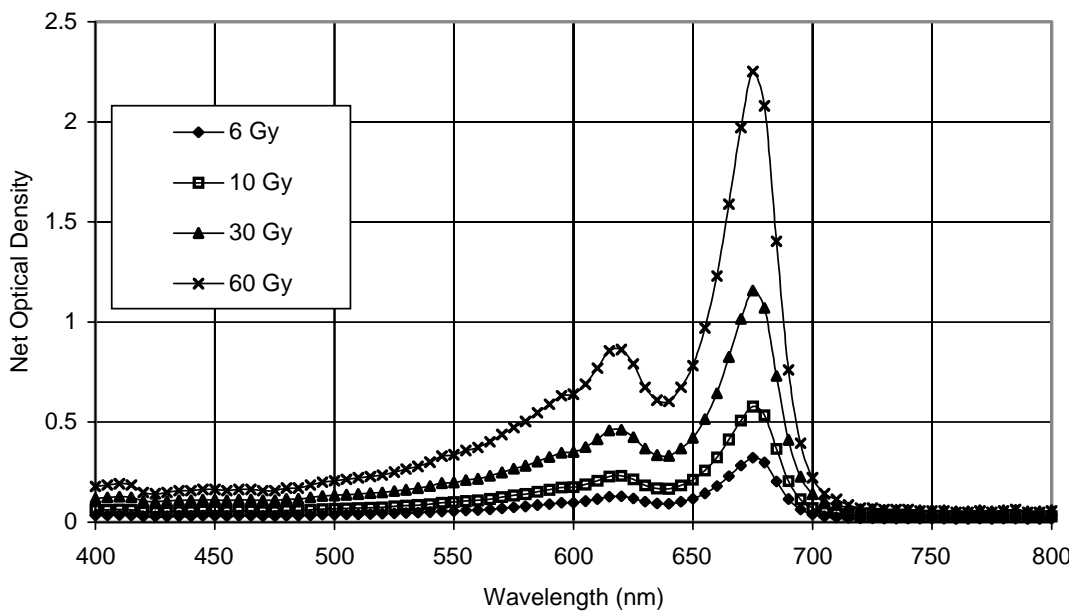

Fig. 6. Net optical density (OD) of an irradiated MD-55-2 radiochromic film exposed to doses up to 60 Gy. The net OD is the subtraction of the background OD from final OD. 
Optical density response at various wavelength for MD-55-2 Gafchromic film

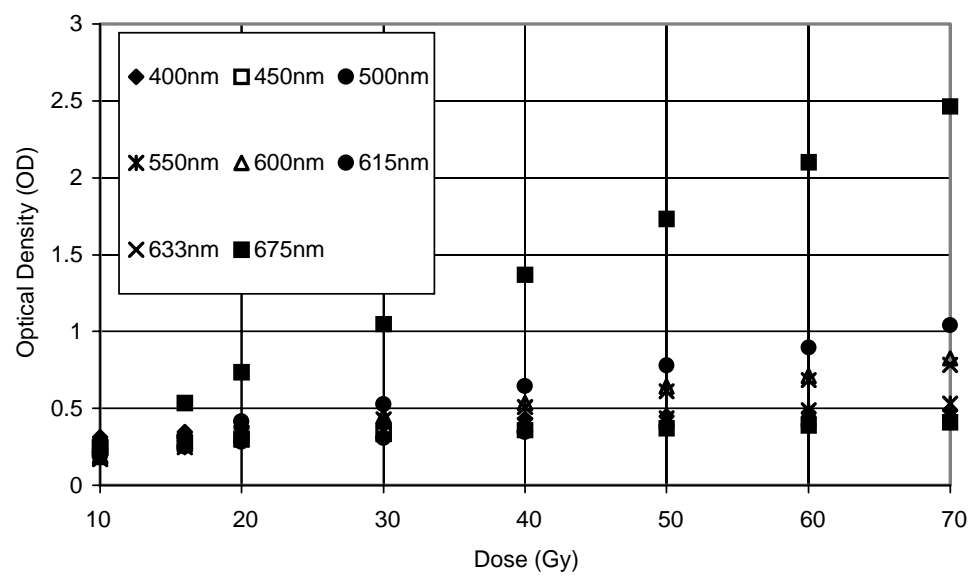

Fig. 7. Dose response of the MD-55-2 radiochromic film when analysed at various wavelengths. Due to the visible absorption characteristics of the radiochromic film, various dose sensitivities can be recorded when analysed at specific wavelengths. Normally, for medical purposes, the maximum dose response is the wavelength of choice to minimize the exposure required for an accurate reading.

absorption peak of $675 \mathrm{~nm}$. The results show a spread of wavelengths with specific points at the two absorption peaks as well as $633 \mathrm{~nm}$, which is the readout wavelength of a helium-neon laser, a device commonly used for densitometry in radiation dosimetry. The response of the film is nearly three times larger at the maximum wavelengths as compared to $633 \mathrm{~nm}$.

\subsection{Dose fractionation}

Radiochromic film has the advantage of being a processless film and colour changes occur without the need for chemical developing and fixing. This advantage makes the film reusable, which is especially useful for fractionated dose deliveries. During radiotherapy treatment using high-energy linear accelerators, doses are delivered in smaller fractions so as to maximise the tumour dose delivery whilst sparing normal tissue in the process [43-45]. Dose measurements have been made to ascertain the effect of dose fractionation on the response of the GAFCHROMIC ${ }^{\circledR}$ MD-55-2 film [46] and other film types [47-49]. The initial densities of various films were measured and various dose regimens were delivered to the film for analyses. Manufacturer testing for fractionation effects is given in Table 2 and has shown that the MD-55-2 film produces a minimal effect depending on the regimen used for dose delivery [46]. The films were given a total exposure of $40 \mathrm{~Gy}$ with $120 \mathrm{kVp}$ $\mathrm{X}$-rays filtered through $2 \mathrm{~mm}$ aluminium. One sample received the total $40 \mathrm{~Gy}$ in a single exposure over a $10 \mathrm{~min}$ period. The other sample received a fractionated treatment of 5-8 Gy doses each given $30 \mathrm{~min}$ apart. The samples were remeasured $24 \mathrm{~h}$ after exposure. The net optical density quoted is the subtraction of the unirradiated OD from the exposed OD. Within experimental and measurement

Table 2

Dose fractionation effects for GAFCHROMIC ${ }^{\circledR}$ MD-55-2 film lot \#J1426-MD-55

\begin{tabular}{llll}
\hline Total dose $(\mathrm{Gy})$ & Number of fractions & No. of measurements & Net density change \\
\hline 40 & 1 & 15 & 1.14 \\
40 & 5 at $30 \mathrm{~min}$ & 15 & 1.15 \\
\hline
\end{tabular}

Courtesy of David Lewis, ISP Technology. 
Table 3

Dose rate dependence of GAFCHROMIC ${ }^{\circledR}$ MD-55-2 film

\begin{tabular}{llc}
\hline Dose rate $\left(\mathrm{Gy} \mathrm{min}^{-1}\right)$ & Average density $\left(\times 10^{-1} \mathrm{~Gy}\right)$ & Deviation from mean $(\%)$ \\
\hline 4.00 & 0.322 & 1.6 \\
3.422 & 0.310 & -2.1 \\
2.00 & 0.318 & 0.6 \\
0.334 & 0.320 & 1.1 \\
0.003 & 0.320 & 1.1
\end{tabular}

Courtesy of David Lewis, ISP Technology.

errors, the results show that the single and fractionated exposures are indistinguishable and demonstrate that dose fractionation effects are absent for the MD-55-2 film.

\subsection{Dose rate}

The effects of dose rate of delivery on the response of MD-55-2 has been tested and it has been

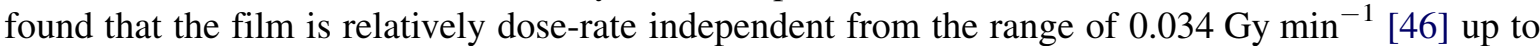
$80 \mathrm{~Gy} \min ^{-1}[50,51]$. Table 3 shows a sample of results for various dose rates taken from different investigators. In each case, the applied dose delivered was either 10 or $20 \mathrm{~Gy}$ at the dose rates designated. Results quoted are the dose rate, average net density change per $10 \mathrm{~Gy}$ and the deviation from the average per group. The net densities differ by less than $\pm 2 \%$ from the mean.

These differences lie within experimental errors of measurements and thus indicate that the film response is dose-rate independent. McLaughlin et al. [41] did find a small dose-rate dependence for the MD-55-2 film when doses larger than 60 Gy were delivered. At this relatively high dose, the investigators quoted an approximately $10 \%$ greater response at the lowest dose rate $\left(0.08 \mathrm{~Gy} \mathrm{~min}{ }^{-1}\right)$ compared to the highest dose rate $\left(80 \mathrm{~Gy} \mathrm{~min}^{-1}\right)$. However at clinical dose rates used for most medical treatments $\left(2-4 \mathrm{~Gy} \mathrm{~min}^{-1}\right)$, the MD-55-2's response is independent of the dose rate. Fig. 8 highlights the dose-rate results for high-energy X-ray beams from dose rates of 0.8 up to $4 \mathrm{~Gy}$ min $^{-1}$ delivered by a Varian $2100 \mathrm{C}$ linear accelerator. As shown, there is negligible dose-rate dependence.

Dose rate dependance of MD-55-2

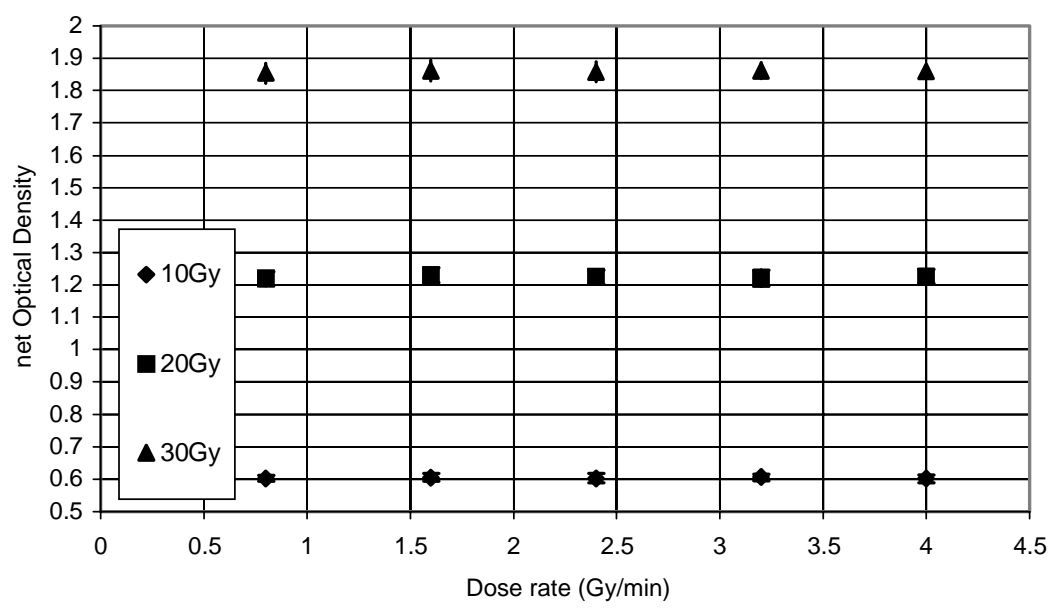

Fig. 8. Dose rate dependence of a radiochromic film at typical medical linear accelerator dose rate and level deliveries. 
Post exposure growth for MD-55-2 Gafchromic film

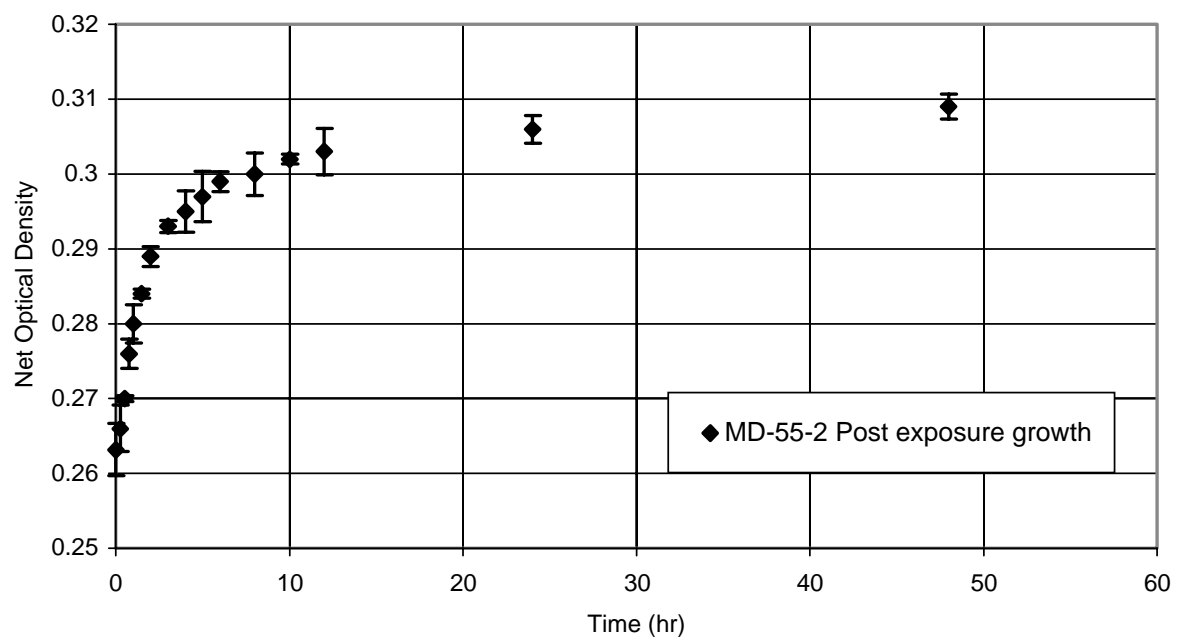

Fig. 9. Post-irradiation optical density growth caused by the continuation of chemical reactions after irradiation has finished. Post-irradiation density growth for most types of radiochromic films is largest over the first few hours with the films relatively stable 1 day after irradiation left at room temperature.

\subsection{Post-irradiation colouration}

The active components in the GAFCHROMIC ${ }^{\circledR}$ MD-55-2 film is a radiation-sensitive monomer. Upon irradiation, the active layer polymerises to form a polymer coloured dye. The work by McLaughlin et al. [52] showed that the reaction has an incubation period of at least $1 \mu$ s. Furthermore, polymerization proceeds after irradiation has ceased causing a post-exposure density growth which manifests itself as a significant increase in the optical absorption. This corresponds to an increased amount of formed polymer in the active layer. The rate of change in this post-exposure increase in the optical absorption does however diminish rapidly with time and the optical absorption seems to asymptote to a constant value approximately $48 \mathrm{~h}$ after exposure has finished $[38,39,42,53,54]$. Fig. 9 shows the post-irradiation growth of the optical density for the MD-55-2 film for various delivered absorbed doses after exposure has ceased. As can be seen in the figure, a significant increase occurs over the first few hours and the film becomes relatively stable after approximately $24-48 \mathrm{~h}$.

This has been confirmed by many investigators [55-58]. As can be seen in Fig. 10, which shows the normalised post-exposure optical density, an approximate 14-16\% increase in the optical density occurs within the first $24 \mathrm{~h}$ after exposure. These results were measured at $660 \mathrm{~nm}$ wavelength.

It is noted by McLaughlin that the post-exposure colouration can vary depending on the wavelength of readout with the main absorption peaks providing a more stable colour density. At $400 \mathrm{~nm}$, colour changes of up to $16 \%$ over the first $24 \mathrm{~h}$ followed by $4 \%$ over the next 2 weeks [38] have been observed. Most plastic dosimeters tend to be unstable in their response during storage periods between irradiation and analysis as discussed by Nablo et al. [59], Uribe et al. [60] and McLaughlin [53]. No appreciable colour change was noticed over a period of 40-165 days when measured at $400 \mathrm{~nm}$ wavelength. It has also been noted that the greatest increase in absorbance occurs at higher storage temperatures such as $40^{\circ} \mathrm{C}$ [37]. Such an effect would need to be taken into account if dosimetry was to be performed at a given time interval after irradiation but before the majority of post-exposure colouration had been performed. Generally, at absorption bands near the 


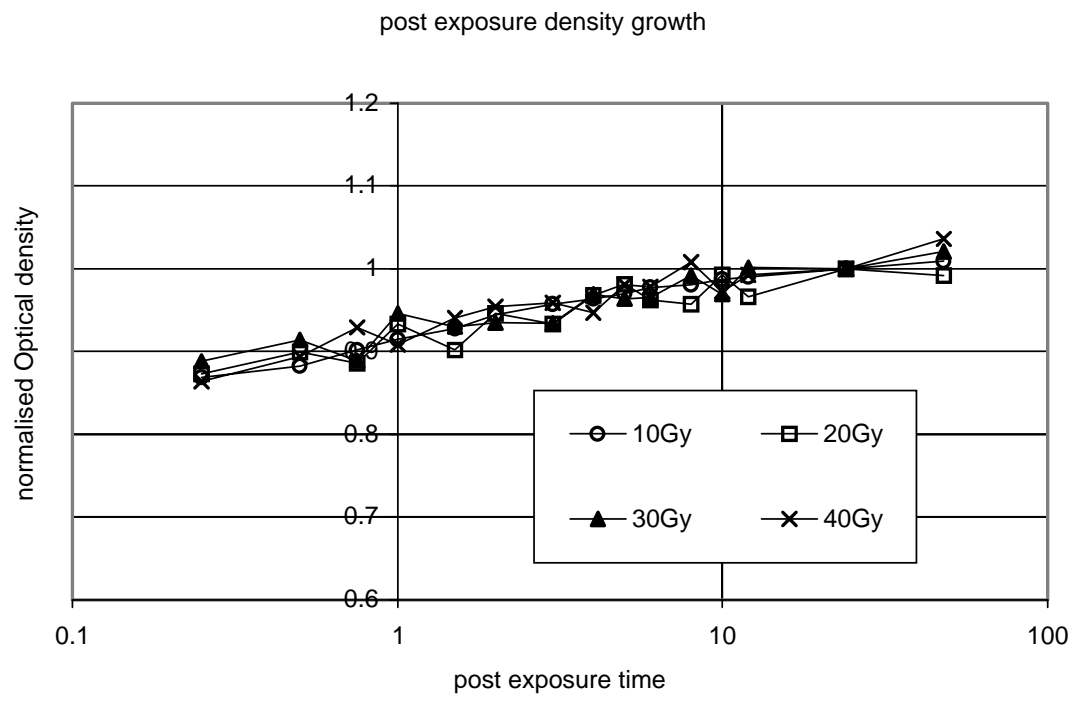

Fig. 10. Post-irradiation growth on a logarithmic scale showing the logarithmic nature of post-irradiation colouration.

main absorption peak, the post-exposure colouration is relatively stable following the first $4 \mathrm{~h}$ after irradiation [49,56]. Various techniques are available to minimize the effects of post-exposure colouration on dosimetry including timing of readings, waiting for extended periods of time and heating techniques on the film to speed the process, which will all be dealt with later in the review.

\subsection{Uniformity in film response}

An ideal film dosimeter will provide a uniform response over a two-dimensional area when exposed to radiation. This allows an easy calibration and dose assessment to be performed without the need for an elaborate position dependent sensitivity correction method. There are two areas of concern for uniformity when using radiochromic films for dosimetry purposes. These can be defined on "microscopic" and "macroscopic" scales. We refer to microscopic uniformity as the degree of fluctuations in the optical density (compared to an average optical density) on the film at one point of interest. The degree of the fluctuations can be caused by many factors including the optical structure of the film active layer and other components, defects in the film structure as well as scratches or other foreign materials such as dust particles. Besides, factors such as the electron noise of scanning densitometers can influence the microscopic uniformity. The microscopic uniformity variations caused by these factors are then often a function of the reader's spatial resolution. In other words, a higher spatial resolution can cause a microscopic variation to appear larger. For example, a black speck of dust $1 \mu \mathrm{m}$ in diameter on the radiochromic film may be insignificant when scanning the film with a spatial resolution of $1 \mathrm{~mm}$ as the result at a "point" is averaged over an $1 \mathrm{~mm}$ region. However, it becomes a major factor for a high spatial resolution such as $10 \mu \mathrm{m}$ resolution. As such, the degree of microscopic uniformity can be expressed by means of the standard deviation in the film response. Fig. 11a-d shows results for a single piece of MD-55-2 radiochromic film exposed to a 20 Gy dose and scanned at resolutions of 300, 150, 75, 60 pixels in. ${ }^{-1}$.

The four different profiles are in the same area on the film. However, the higher resolutions show larger degrees of microscopic non-uniformity or higher standard deviations. The results show that the variations in microscopic uniformity are due to the spatial resolution. The second type of 

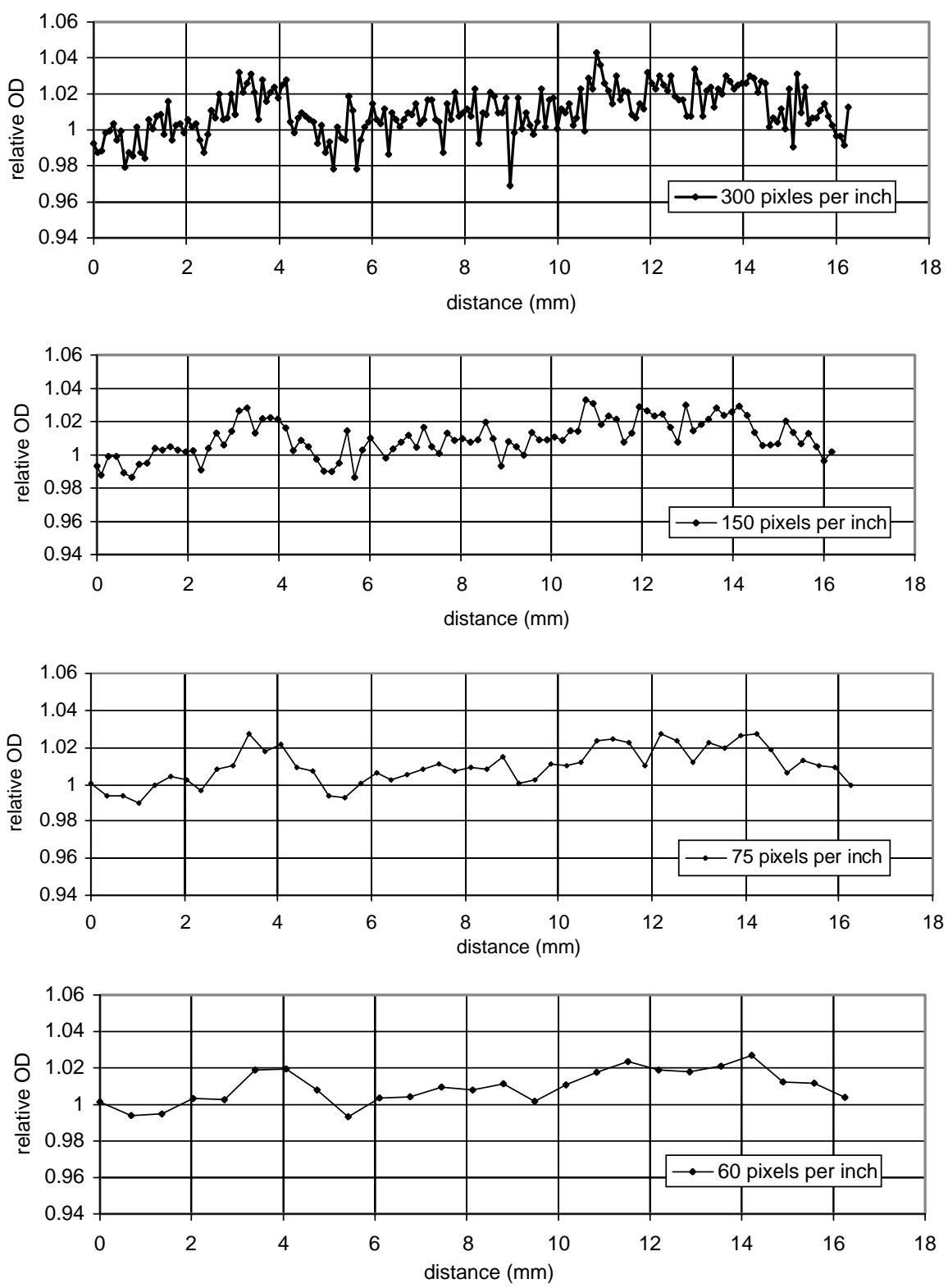

Fig. 11. Microscopic film non-uniformity. Variations in the measured optical density as a function of spatial resolution, highlighting the nature of "pixel" averaging. Resolutions of scanning are 300, 150, 75, 60 pixels in. ${ }^{-1}$ for a, b, c and d, respectively.

non-uniformity, which we will call the macroscopic uniformity, can be caused by variations in the physical or chemical properties of the active layer of films or by systematic variations of the densitometer used. It can be ascertained or often seen on a larger scale of spatial magnitude producing global maximum and minimum outputs in the measured optical density where a uniform radiation dose has been delivered. The macroscopic uniformity of various types of radiochromic films has been investigated by researchers and can vary from batch to batch. The original MD-55-1 single-layer film produced by ISP Technology has been reported to have variations up to $15 \%$ for macroscopic uniformity [57] and this was not seen in a predictable pattern. Butson and Yu [61] also 


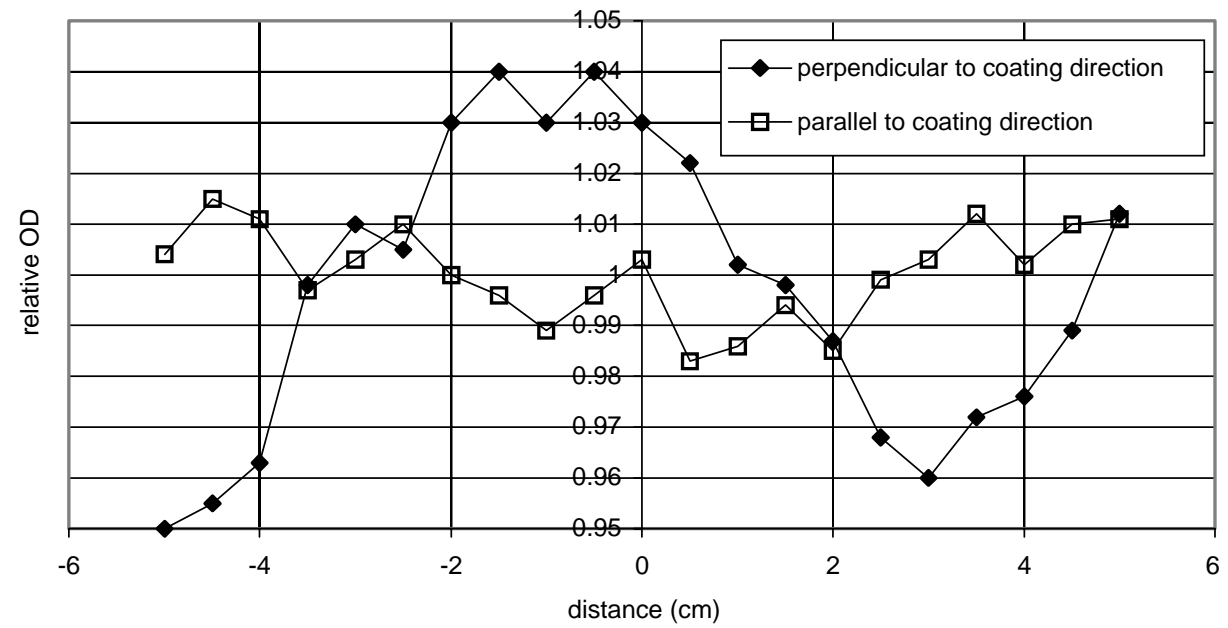

Fig. 12. Film optical density uniformity for the MD-55-1 radiochromic film, both perpendicular and parallel to coating direction. Larger variations were seen in older film products.

found that the MD-55-1 could produce a non-uniformity of less than $2.5 \%$ and that the size of the variation was batch dependant. Meigooni et al. [58] also found large variations but reported that a large degree of variation could be attributed to the coating direction of manufacturers (up to $15 \%$ ) and up to $4 \%$ in a direction parallel to the coating. This is expected to be due to the manufacturing process and future products (MD-55-2) and MD-HS have kept macroscopic non-uniformity to less than $6 \%$ overall. To distinguish the coating direction, manufacturers now produce a marking system to show the coating direction to provide researchers the knowledge of the more uniform direction. Fig. 12 shows variations in the uniformity for MD-55-1 for two different sheets in the parallel and perpendicular directions of coating.

The scale shows the relative optical density compared to a specific point on the film. The macroscopic variations are quite noticeable on one sheet but are relatively small on the other. This was inherently due to the manufacturing process where the MD-55-1 active layer was coated onto the base film without a controlled gauging mechanism in place to minimize the effects of active layer thickness variation. Fig. 13 shows the same results for a MD-HS film. The macroscopic uniformity has been improved with the manufacturer quoting a maximum variation of $6 \%$ non-uniformity in any direction.

Microscopic and macroscopic uniformity variations can influence the results required for dosimetric applications. However, an acceptable tolerance level for non-uniformity will depend on the level of accuracy required for the given application. In most radiotherapy, an acceptable level of variation would be considered to be in the order of +3 to $5 \%$, which are often the levels produced by other film media such as radiographic emulsions. To perform a uniformity check on a radiochromic film, a uniform radiation field is required. This is normally delivered by a large field from a medical linear accelerator whereby a flatness variation would normally be of the order of less than $2 \%$ over a $10-15 \mathrm{~cm}$ range. As many investigators require a two-dimensional film dose result [62-66], any nonuniformity must be minimal or at least be able to be corrected for.

\subsection{Double exposure techniques for minimising macroscopic non-uniformity}

When a macroscopic non-uniformity exists and would be considered significant enough to affect the accuracy and reproducibility of results, various methods can be used to minimize this 


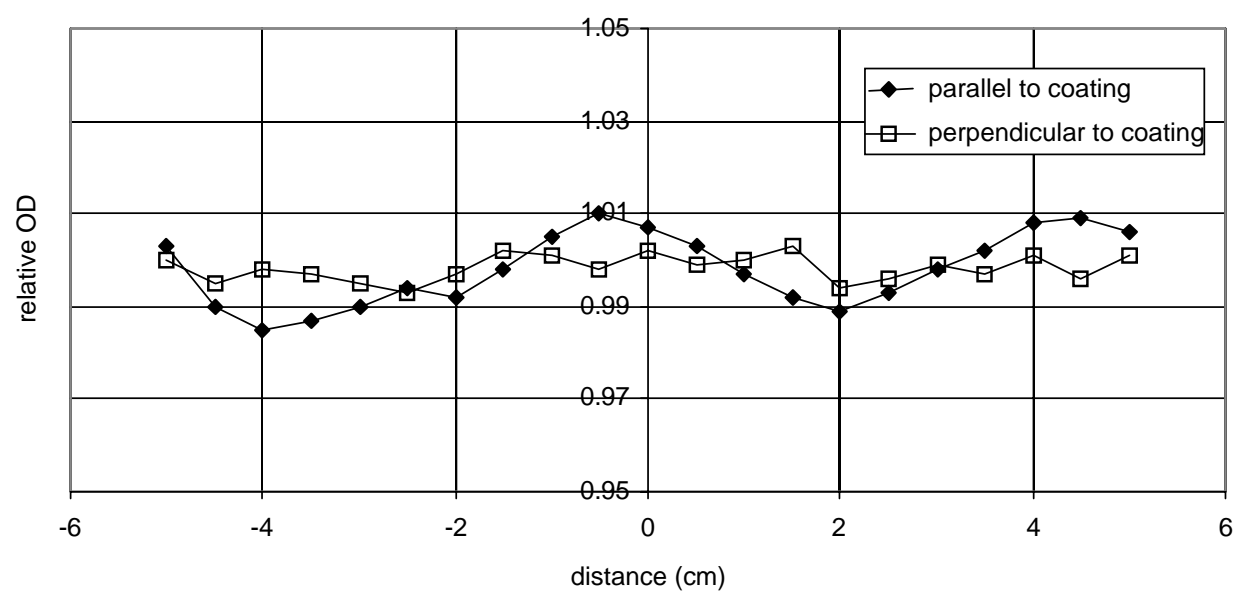

Fig. 13. Typical film uniformity for newer, revised versions of radiochromic films. This film is GAFCHROMIC ${ }^{\mathbb{R}}$ HS $_{\text {with }}$ variations shown in parallel and perpendicular to the coating directions as well. Improvements to manufacturing techniques have improved the quality of commercially available radiochromic film products.

effect. A relatively simple procedure is the use of what is commonly called a double exposure technique [57,66-68]. In this process, a sensitivity value is assigned to each section of the radiochromic film, which is due to its initial starting optical density and its sensitivity at each section. This can be normalised to a film average with the end result producing a normalised response over the entire film section. This process is used for many types of dosimeters, which are used in a batch or group situation such as thermoluminescent dosimeters [69-72] and diode arrays [73-76]. Zhu et al. [57] initially reported this process for the radiochromic film due to the relative independence of films on fractionation regimen. That is, an initial dose can be delivered causing an optical density change without any significant effect on future OD to dose properties. In the double exposure technique, a matrix of sensitivity corrections are obtained over a two-dimensional area of the film exposed in a uniform field. The size and spatial resolution of this matrix depends on the resolution of the reader used or the spatial resolution required for the experimental work. The film can be marked with a series of fiducial markers (normally a minimum of three) and the film exposed to a known uniform dose $\mathrm{D} i$. The average optical density of the film $\mathrm{OD} i_{(x, y)}$ in the area of interest can be determined after a known post-irradiation colouration period. The change in OD for the region of interest produced by the known dose provides a unique sensitivity value for the film represented by

$$
S_{(x, y)}=\frac{\mathrm{OD} i_{(x, y)}-\mathrm{OD}_{(x, y)}}{\mathrm{OD} i_{(x, y)}-\mathrm{OD} 0_{(x, y)}},
$$

where OD0 is the background fog OD for the film.

Following the calibration process, the film can be exposed to the experimental unknown dose $\mathrm{D} j$. Then the corrected OD reading becomes

$$
\mathrm{OD}_{\text {net }} \operatorname{corr}_{(x, y)}=\frac{\mathrm{OD} i_{(x y)}-\mathrm{OD} j_{(x y)}}{S_{(x y)}} .
$$

This type of two-dimensional sensitivity correction is easily applied with computer-controlled software analyses and the correct alignment of the fiducial markers. 


\subsection{Ambient and readout light source effects}

Most radiochromic films are sensitive to ultraviolet radiation [77-81] in varying degrees producing colouration, which may be used specifically for ultraviolet radiation dosimetry or cause unwanted reactions during X-ray or $\gamma$-ray dosimetry. For most medical, nuclear and industrial dosimetry, ultraviolet light produces an unwanted colouration of the film and appropriate handling and storage conditions are required. This includes protection from ultraviolet sources such as the sun and fluorescent light exposure, and the films should thus be stored in an opaque container and only taken out for experiments and readout. The magnitude of the ultraviolet reaction depends on the wavelength and source intensity of the ultraviolet radiation. For natural background effects, various studies have been performed by investigators to quantify the influence of sunlight and fluorescent light sources [56,82-86]. One method of showing the effects of ambient light is by 'dose equivalent colouration', i.e. the amount of light exposure which produces a similar OD change to the radiochromic film as a uniform X-ray dose field. Fig. 14 shows examples of the effects from various ambient light source exposures in producing results for equivalent dose colouration. The light sources exposing the film in this figure were as follows.

Sunlight: Both full direct sunlight and filtered sunlight during spring time in New South Wales, Australia were used. The filtered sunlight was through $50 \%$ black plastic shade cloth. The luminance during the experimental procedure was approximately $40 \mu \mathrm{W} \mathrm{cm}{ }^{-2} \mathrm{~nm}^{-1}$ for direct sunlight and $25 \mu \mathrm{W} \mathrm{cm} \mathrm{nm}^{-1}$ for filtered sunlight.

Fluorescent light: Fluorescent light sources were two $40 \mathrm{~W}$ fluorescent tubes at a distance of $1.5 \mathrm{~m}$. The filtered fluorescent light had a $3 \mathrm{~mm}$ plastic diffuser cover as would be the case in most office conditions. The visible luminance was $0.64 \mu \mathrm{W} \mathrm{cm}{ }^{-2} \mathrm{~nm}^{-1}$ for direct fluorescence and $0.59 \mu \mathrm{W} \mathrm{cm}{ }^{-2} \mathrm{~nm}^{-1}$ for the diffused fluorescence.

Incandescent light: Incandescent light was a $60 \mathrm{~W}$ bulb at a distance of $1.8 \mathrm{~m}$ with a visible luminance of $0.085 \mu \mathrm{W} \mathrm{cm} \mathrm{cm}^{-2}$.

The results show that the largest effects are seen with direct sunlight per unit time, which is due to the high luminance output. Fluorescent lights also produced a significant colouration with a

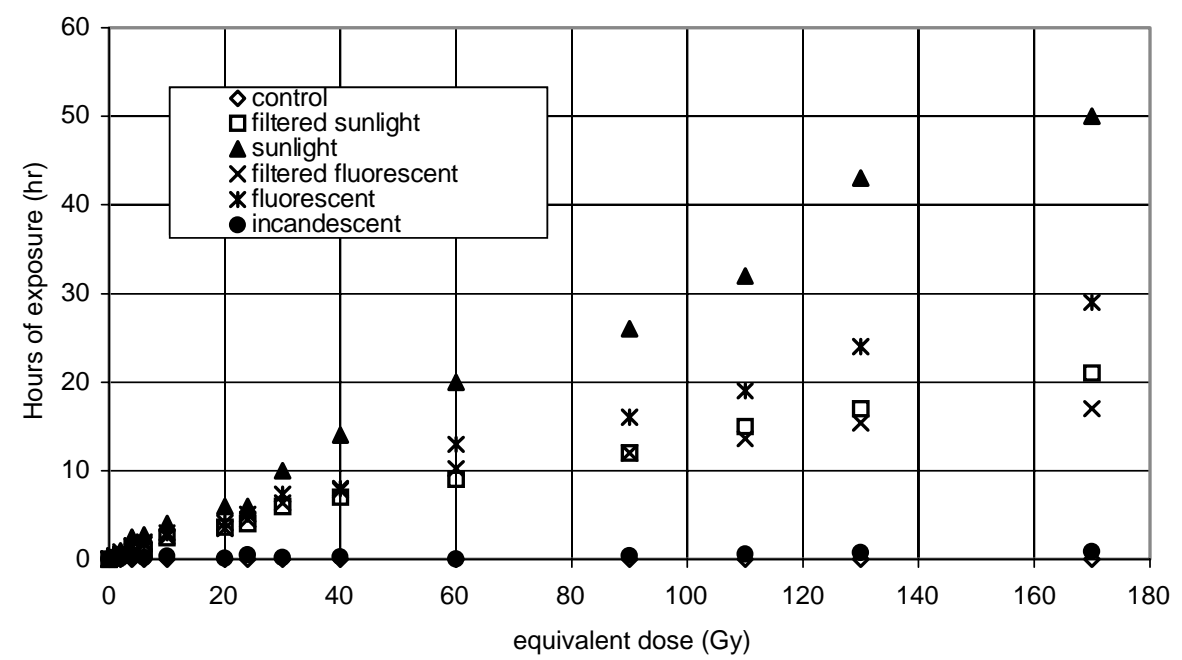

Fig. 14. Effects of ambient light sources on the coloration of radiochromic film products. Both sunlight and fluorescent light sources can effect the coloration of radiochromic film due to the ultraviolet content in their beam intensity. Shown in this figure is the response of the MD-55-2 film. 
20-30 Gy equivalent dose after $175 \mathrm{~h}$, while incandescent lights produced a negligible effect [78]. Probably the most significant are the results in the effects of fluorescent ambient light sources which are commonly used in all major institutions for lighting purposes due to their relatively low-energy requirement for running. At the measured distances, which equate to an average distance the films would be from fluoro lights during use in office or laboratory-type buildings, an equivalent dose exposure of $10-20 \mathrm{cGy} \mathrm{h}^{-1}$ is recorded. This could be considered a low-level effect when measurements of 20-50 Gy are common. However, results do show that extended periods of fluorescent light exposure (due to their UV content) can have a significant effect of dosimetric accuracy of colouration of the film. This reiterates the recommended requirement of keeping the film in an opaque container when not in use. Our recommendation is that experiments and handling in fluorescent room light is acceptable but the time spent with film unwrapped should be minimised.

Readout light sources for densitometry can also have an effect on the radiochromic film if there is a UV component to the source. This can be of concern due to the close proximity of the radiochromic film to the light source. Fig. 15 shows the effects of various commonly used readout light sources on the MD-55-2 radiochromic film. These commonly used light sources are: (1) helium-neon lasers; (2) ultra-bright red light emitting diodes (LEDs); and (3) fluorescent lights. Densitometric readout systems for radiochromic film analysis is examined in more detail in another section of this review. Results from the figure were derived using the following light sources. A $1 \mathrm{~mW}$ helium-neon laser with wavelength $632.6 \mathrm{~nm}$ was employed, and the film was placed $3 \mathrm{~mm}$ from the beam aperture and the beam diameter was $1 \mathrm{~mm}$. A red GaAlAs 300 mcd ultra-bright LED in clear plastic housing (peak wavelength $660 \mathrm{~nm}$, according to manufacturer specifications). Again, the film was placed $3 \mathrm{~mm}$ from the front tip of the LED. A $40 \mathrm{~W}$ fluorescent light source was used with the film placed $5 \mathrm{~cm}$ from the edge of the tube. Each configuration was designed to simulate standard conditions for densitometry work. Design variations in densitometers and scanners will provide a range of conditions. The above configurations show a sample of effects possible.

The results show that care must be taken when using fluorescent light sources for readout of radiochromic film products. After $3 \mathrm{~h}$ of exposure, an equivalent dose colouration of $20 \mathrm{~Gy}$ can be seen. This equates to an equivalent dose colouration of $11 \mathrm{cGy} \mathrm{min}^{-1}$. As a typical scan using a

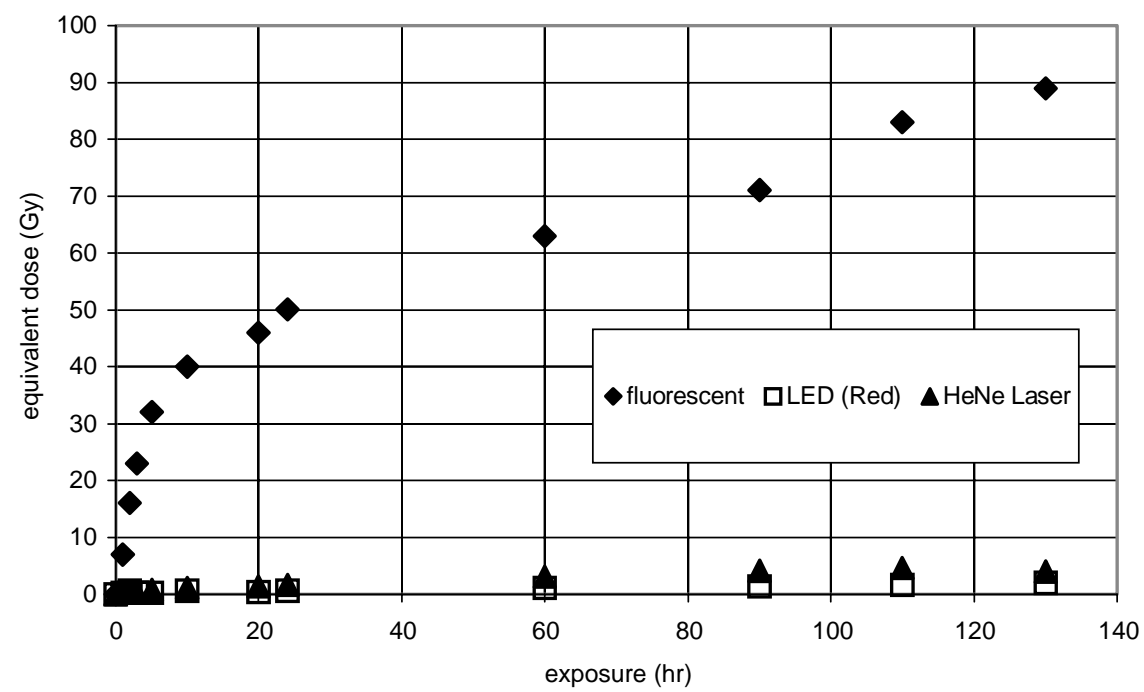

Fig. 15. Effects of readout light sources on the coloration of radiochromic film products. Fluorescent light sources and laser light sources can affect the coloration of radiochromic films due to the ultraviolet content in their beam intensity. Shown in this figure is the response of the MD-55-2 film. 
densitometer takes approximately 10-20 s, a dose equivalent colouration in the order of less than $10 \mathrm{cGy}$ would be seen. However, due to the nature of some scanning densitometers, the films can be partially "left in" the scanner during image reconstruction and analysis stages. Depending on the time required for these steps, the end result can be a significant effect on the optical density of the film. Visible LEDs produce a negligible effect of radiochromic colouration whilst the helium-neon laser produced a small quantifiable effect due to the UV components within its emission spectra.

\subsection{Ultraviolet dosimetry}

As radiochromic film is sensitive to ultraviolet radiation, potential arises for quantitative dosimetry to be performed. Many types of detectors are used for ultraviolet dosimetry including broad-band radiometers [87,88]. The biological effective irradiance can be determined if suitable filters and sensors are selected to provide responses similar to the action spectrum of the biological material in question. Several passive detectors [89-91] have been developed for different ultraviolet dosimetry purposes. Polysulphone film has been extensively used for measurements of human exposure to UV due to the fact that its action spectrum (spectral response) closely matches that of the human epidermal tissue. Radiochromic film was used to quantitatively measure ultraviolet radiation [80,92-94] and it was found that, for the MD-55-2 film, the response was predominantly in the UVA waveband. Measurements were performed using various ultraviolet, visible and infrared sources from a solar simulator allowing simulation of the full spectrum of terrestrial sunlight at the equator at mid-summer noon and variable through all wavelengths. Fig. 16 shows the normalised response to various parts of the UV, visible and infrared spectra.

The predominant response in the UVA region is due to the design characteristics of the MD-552 film. The MD-55-2 active components are protected by an outer plastic coating which absorbs ultraviolet radiation in the UVB waveband thus causing the negligible response. MD-55-2 film was also shown to be used quantitatively for UVA exposure with both fluorescent light sources and solar radiation as shown in Fig. 17.

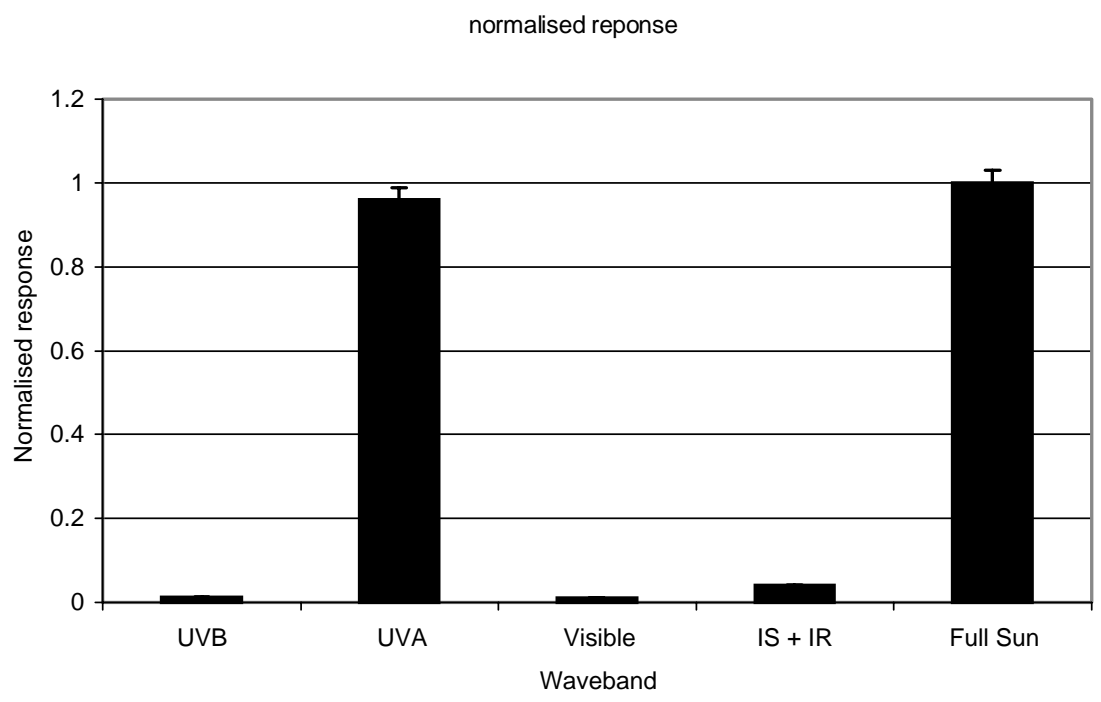

Fig. 16. Response of the MD-55-2 radiochromic film to ultraviolet, visible and infrared radiation sources. Films were exposed with a solar simulator, which contained spectrum contents matched to solar radiation. The film is most sensitive to UVA wavebands. 


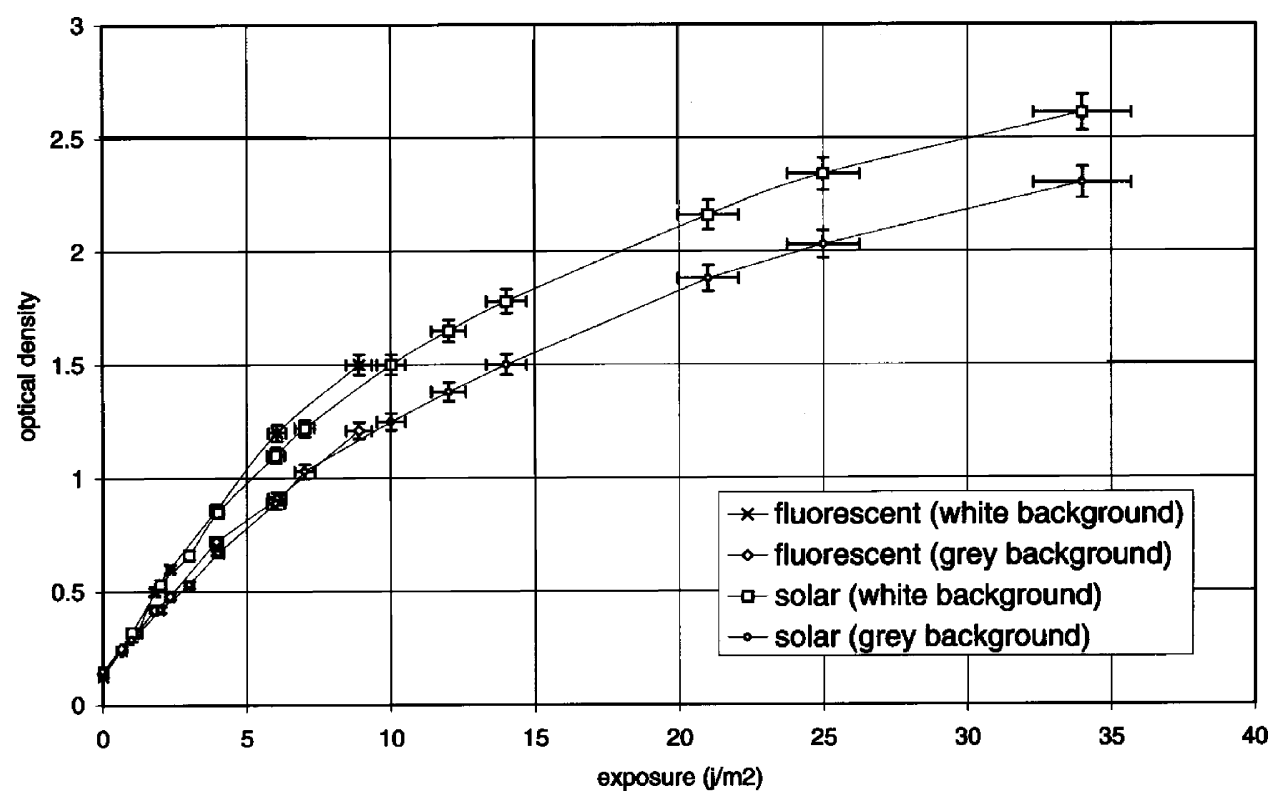

Fig. 17. Exposure response of the MD-55-2 radiochromic film to fluorescent light produced ultraviolet and solar produced ultraviolet radiation.

The optical density response was measured at the wavelength $660 \mathrm{~nm}$ and the results showed that a second-order polynomial fit could be applied at this wavelength. Similar sensitivities were recorded for fluorescent and sunlight sources, which is due to wavelength response in the UVA region for MD-55-2 film. As most radiochromic film products possess transparent properties, ultraviolet dosimetry can be dependent on the quantity of backscattered UV as well as the incident intensity. These properties were highlighted by Butson et al. [80] in a study where various coloured background materials were used when irradiating with standard UV sources. Table 4 shows the relative normalised response from the film dosimetry with different absorption backgrounds ranging from white, colours and black. The results showed that when normalised to 1 for white background, a black background produced a response of approximately 0.8 or $80 \%$. The UV response is also similar to the X-ray response for film batch variations and double exposure techniques are often performed to avoid non-uniformity problems and batch sensitivity variations.

\subsection{Polarization effects}

Many radiochromic films are produced with various layers in their construction. As an example, GAFCHROMIC $^{\circledR}$ MD-55-2 film is produced with multiple layers of mylar protective coatings,

Table 4

Normalised response of GAFCHROMIC ${ }^{\mathbb{R}}$ radiochromic film to UV exposure with various backing materials

\begin{tabular}{ll}
\hline Colour & Normalised response \\
\hline White & 1.00 \\
Bottle green & 0.89 \\
Sky blue & 0.87 \\
Scarlet red & 0.92 \\
Dark grey & 0.83 \\
Black & 0.81 \\
\hline
\end{tabular}


radiosensitive gels and glue. These types of constructions can produce effects based on the polarization qualities of the readout light sources used for optical density evaluation. Many analysing light sources are polarised to some degree. Light sources can be polarised by reflections off gratings or mirrors or in the case of helium-neon lasers two orthogonally polarised modes can be expected [62]. The amount of polarization, which is recorded from a densitometer, can be measured using a polariser placed at various known angles within the densitometer used. The use of a scanning electron microscope has shown that the micro-crystals in the active layer of the MD-55-2 radiochromic films have a preferred orientation. As MD-55-2 is made from a two-active-layer construction, there is a potential for the plane of polarization of these layers to affect the optical density characteristics of the film as a function of angle to the incident readout light source. The mylar sheets used to construct the film and hold the active layers together can also have polarization properties. Klassen and others have found polarization effects on GAFCHROMIC ${ }^{\circledR}$ MD-55-2 films $[95,62]$. Klassen found variations up to $8 \%$. They have also shown that the orientation of the film in terms of front and back can also produce a variation in the measured optical density on the same piece of film. This is particularly important for calibration films as small film samples (often $1 \mathrm{~cm} \times 1 \mathrm{~cm}$ ) are normally cut and irradiated to the known doses for calibration purposes. It is quite easy to flip or rotate the films before or after irradiation to the wrong direction unless a permanent marker strip is attached to the films. Using a Cary 210 spectrophotometer (Varian Optical Spectroscopy Instruments, Vic., Australia), the degree of variation seen is not only due to the polarization properties of the radiochromic film, but also due to the level of polarised light in the spectrophotometer itself. Klassen also found that with polarised light coming in and a polariser placed behind the radiochromic film, large variations (up to a factor of 2) in the output intensity were found with angular rotations of the film (GAFCHROMIC ${ }^{\circledR}$ MD-55-2, batch no. 941206).

However, not all radiochromic films have polarization properties to this extent. Similar studies have been performed by the authors to evaluate the polarization effects produced by the GAFCHROMIC ${ }^{\circledR}$ HS film. With a $660 \mathrm{~nm}$ LED spot densitometer, the GAFCHROMIC ${ }^{\circledR}$ HS film was rotated through $180^{\circ}$ to check for any polarization effects. Fig. 18 shows the variation in the optical density output normalised to 1 at a reference angle for the film. No rotational effect was observed. With the use of linear polarised light from a $660 \mathrm{~nm}$ red light emitting diode dosimetry

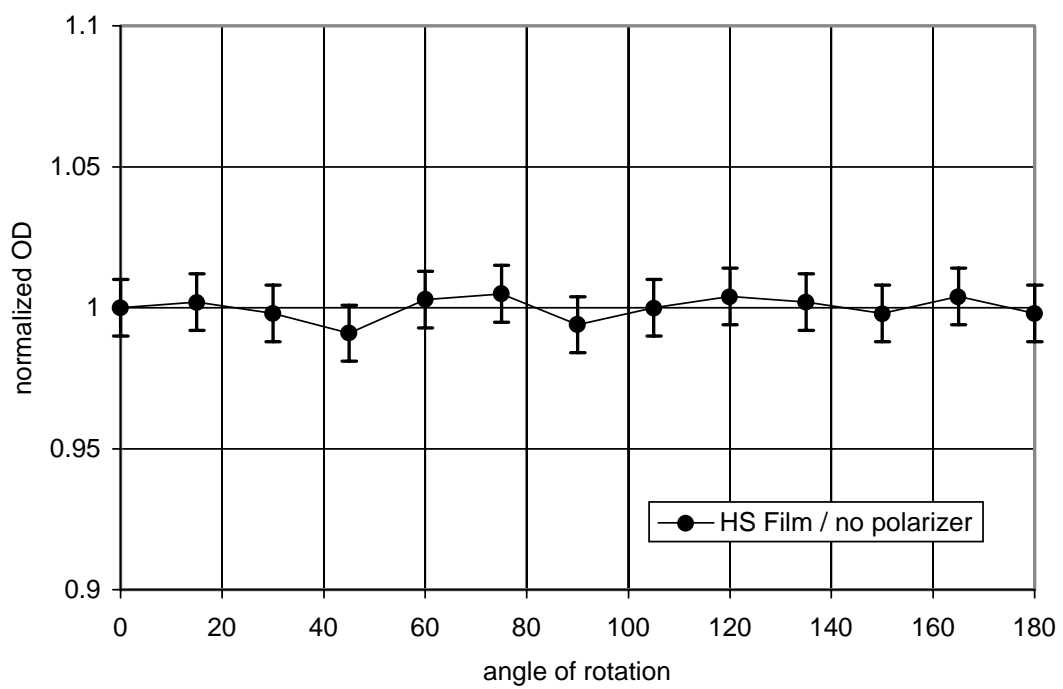

Fig. 18. Polarization response of the HS radiochromic film with unpolarised light source and readout system. 


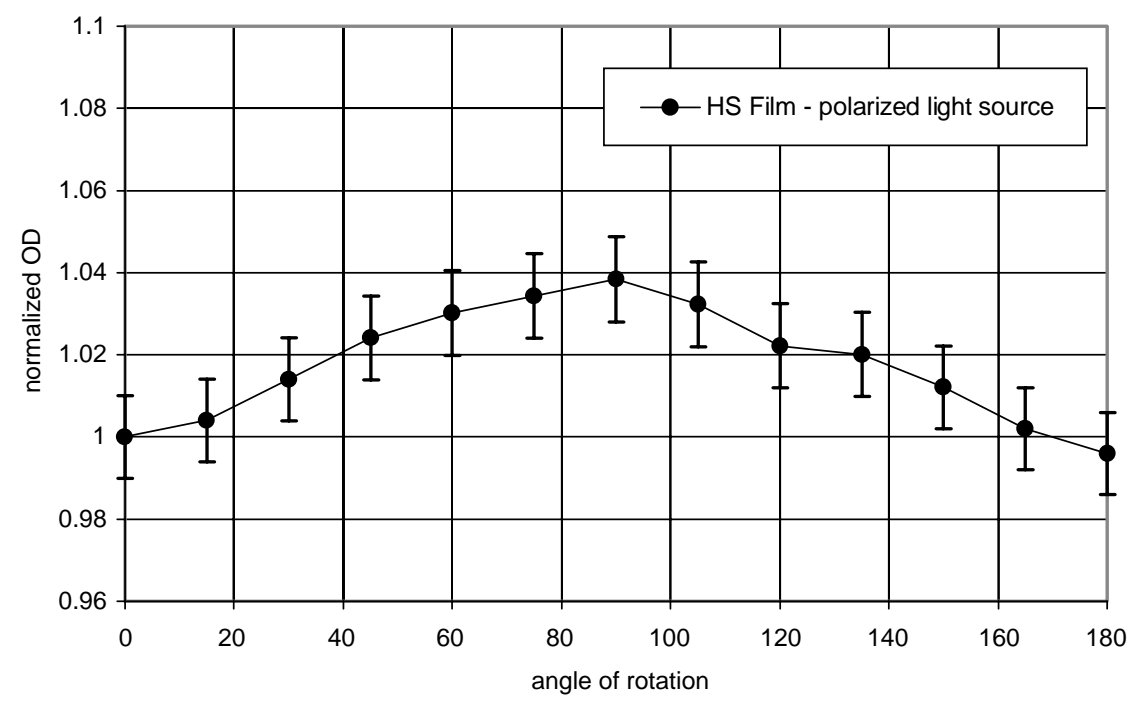

Fig. 19. Effects of polarization with the HS radiochromic film with a polarised light source.

system (polarization performed with a sheet Polaroid), a minimal polarization effect was found. A variation of up to approximately $4 \%$ was seen in output OD when the film was rotated through $360^{\circ}$ as shown in Fig. 19. It is assumed that the removal of the middle mylar layer from the film construction, compared to the older version of MD-55-2 film tested by Klassen, has removed most polarization effects. When both light source and readout detector are polarised, the effects of the film are large. When the polarisers are rotated to allow maximum intensity output without the presence of film, the effects of the film are shown in Fig. 20. The film produces a phase shift in the light between the source and detectors, effectively "rotating" the polarised light to a varying angle, and thus varying the transmitted light intensity. The results shown are normalised optical density [96,97]. Before measurement of radiochromic film with a specific densitometry system, we recommend that a test be performed to evaluate the polarisational properties of the system used. Depending on the

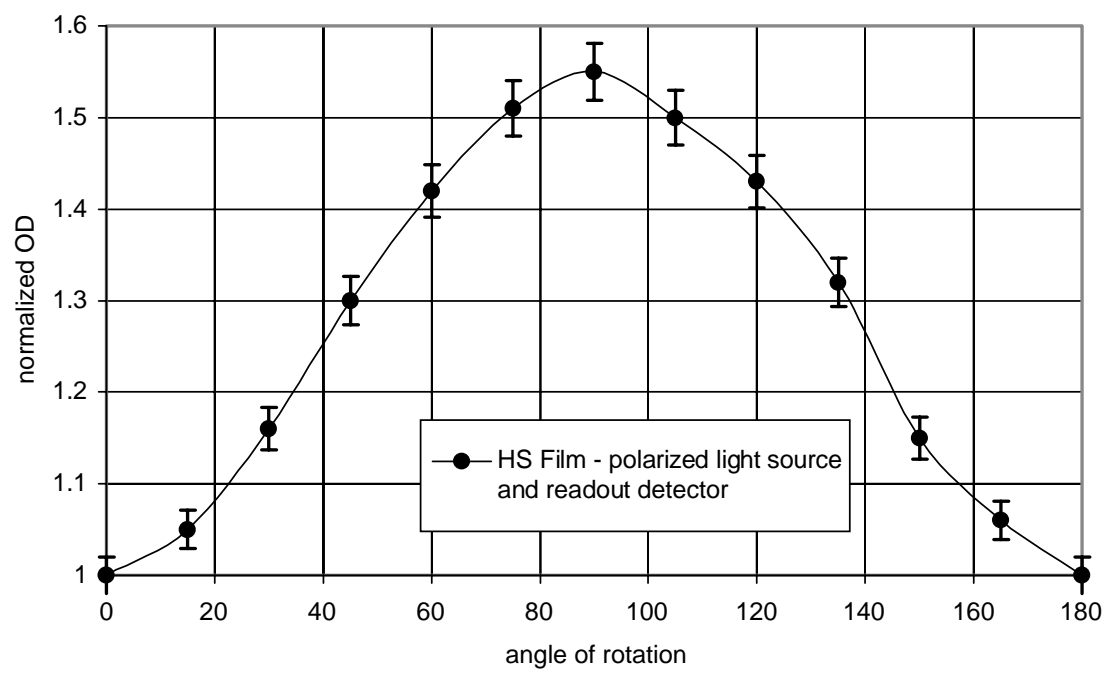

Fig. 20. Dosimetric effects of the HS radiochromic film on visible phase shifting for both polarised light source and readout detector. 
degree of polarization in the light source and the detection system, corrections may have to be performed or more care taken to keep the orientation of the radiochromic film pieces the same throughout the readout process.

\subsection{Interference fringe effects}

Recent studies of transmission scanning densitometers and diode point densitometers have shown that artefacts can be created from interference fringe effects producing diffraction-like patterns such as Newton's rings. These effects are caused by multiple reflections due to changes in the index of refraction along the light beams path. This can be caused by air gaps between the film and a "glass" surface (Newton's rings) or by slight variations in the film thickness which can lead to intra-film interference. To minimize the effects of interference, a clear glass sheet within a film scanner can be replaced by a diffused glass sheet producing an incoherent light source [98]. An example of interference fringes produced is shown in Fig. 21, which is a result of a radiochromic film on a clear glass scanner.

The interference fringes can produce large variations in the measured optical density especially if a selective wavelength of analysis is used. The film, which is normally blue in colour, can have multi-color interference fringes. Fig. 22 shows the variation in the optical density seen across these interference fringes as measured at $660 \mathrm{~nm}$ wavelength. Variations up to $35 \%$ have been recorded.

The introduction of a diffusion filter into a densitometer has been shown by Dempsey [62] to not significantly affect the accuracy or precision of film dosimetry when scanning is performed on irradiated radiochromic films. If scanning is to be performed on a transmission densitometer which

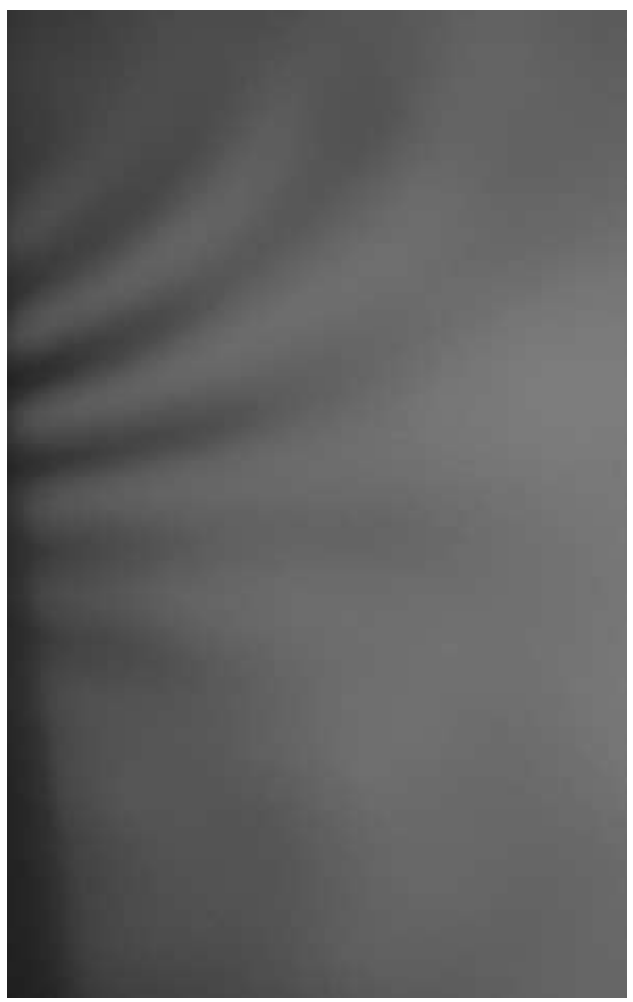

Fig. 21. Interference fringes produced using a radiochromic film. 


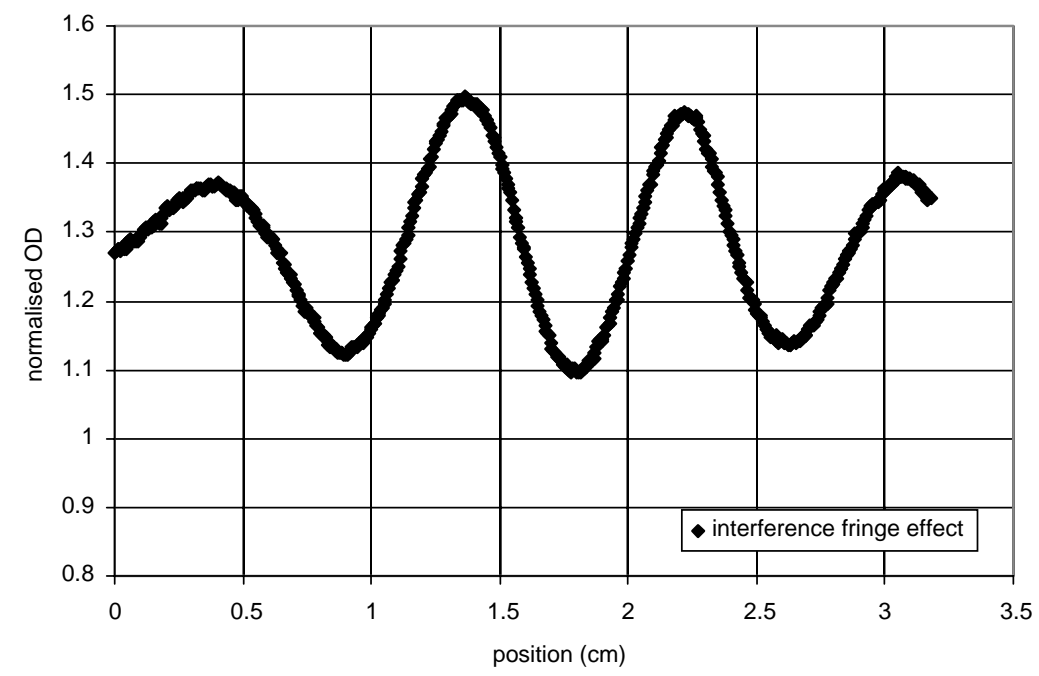

Fig. 22. Optical density profile across an interference fringe region on a radiochromic film affecting absolute dose measurements.

incorporates a reflective glass surface or equivalent, measurements should be made to ascertain the level of interference associated with the scanner. If interference fringes are present, the glass should be replaced with a diffused surface or scanning performed with a calibrated diffuser.

\subsection{Energy dependence of radiochromic film}

Many investigators [58,99-102] have studied the energy dependence of radiochromic films in many applications. For all applications, the degree of energy dependence can affect the dosimetry properties of the film when an unknown spectrum of radiation energies are present. The lower atomic number of most commercial radiochromic films make them more energy independent than silver halide radiographic films which have a large energy dependence due to the high-density materials present. However, a degree of energy dependence does exist in most products under-responding at lower X-ray energies. Muench et al. have compared the variations of the low-sensitivity radiochromic film HD-810 (formally Dm-1260) response along with various other investigations for other radiochromic film products [58,99-102]. The energy dependence of the most well-known and used radiochromic film products are given in the Fig. 23. The energy dependence of these products shows a lower response for low-energy X-rays, which is due to the low atomic number composition of the products. Sayeg et al. [103] have suggested that the lower response of this film is due to the larger carbon content in the film relative to that in soft tissue. Fig. 23a-c show the energy dependence of GAFCHROMIC ${ }^{\circledR}$ MD-55-1, MD-55-2 and HS films to low-energy X-rays, which all show under-responses at lower energies. This is opposite to many other dosimeters used for radiation detectors such as thermoluminescent dosimeters [104-107], silicon diodes [108-110], radiographic films [111-114] and metal oxide semiconductor field effect transistors (MOSFETs) [115-117], where an over-response to low-energy X-rays is seen due to the increased incidence of photoelectron interactions.

Fig. 24 shows these curves relative to the energy response of LiF TLDs and radiographic film (X-omat V). LiF TLDs produce a maximum over-response in the order of approximately $50 \%$ when compared to megavoltage dosimetric evaluations. Detectors such as silver-based radiographic films produce an over-response in the order of 10-12 times (or 1000-1200\%). This shows the ideal 


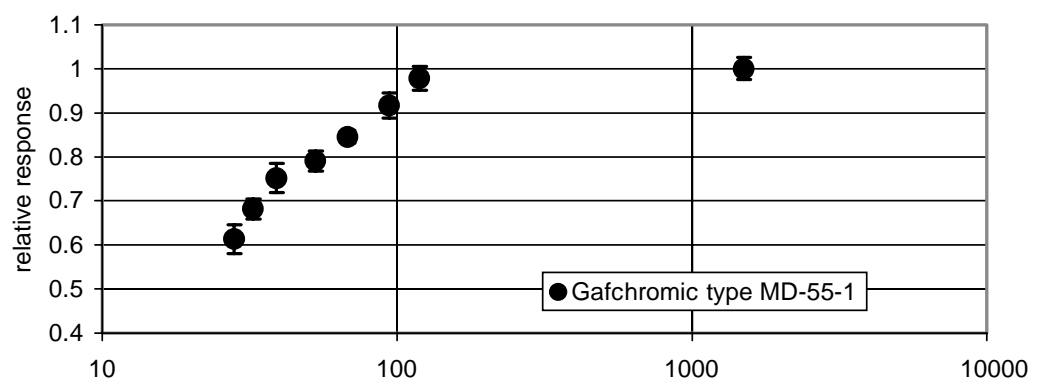

(a)

effective energy (keV)

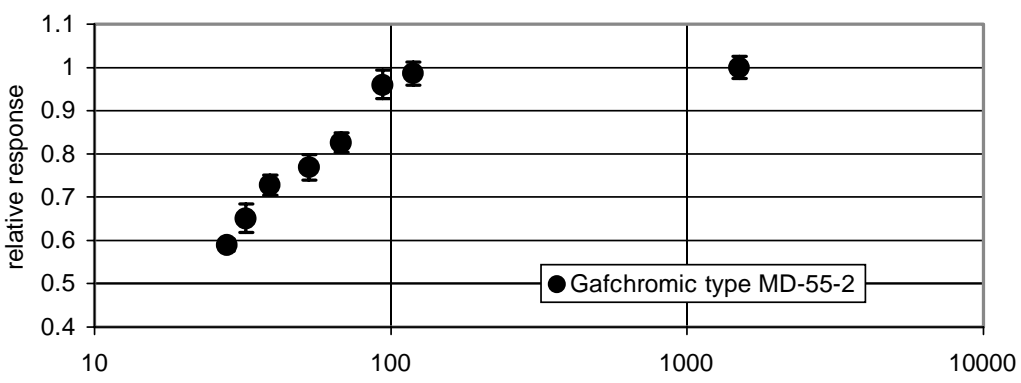

(b)

effective energy (keV)

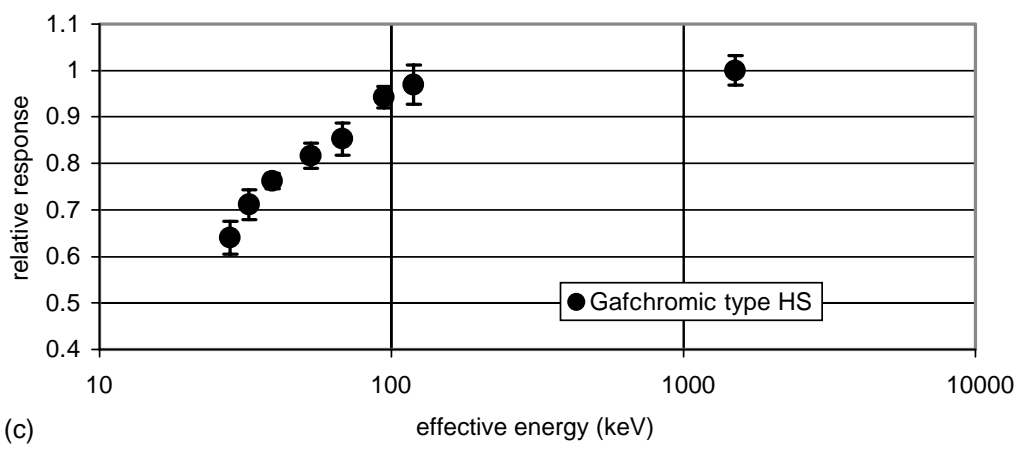

Fig. 23. Energy dependence of (a) MD-55-1, (b) MD-55-2 and (c) GAFCHROMIC ${ }^{\mathbb{B}}$ HS radiochromic films.

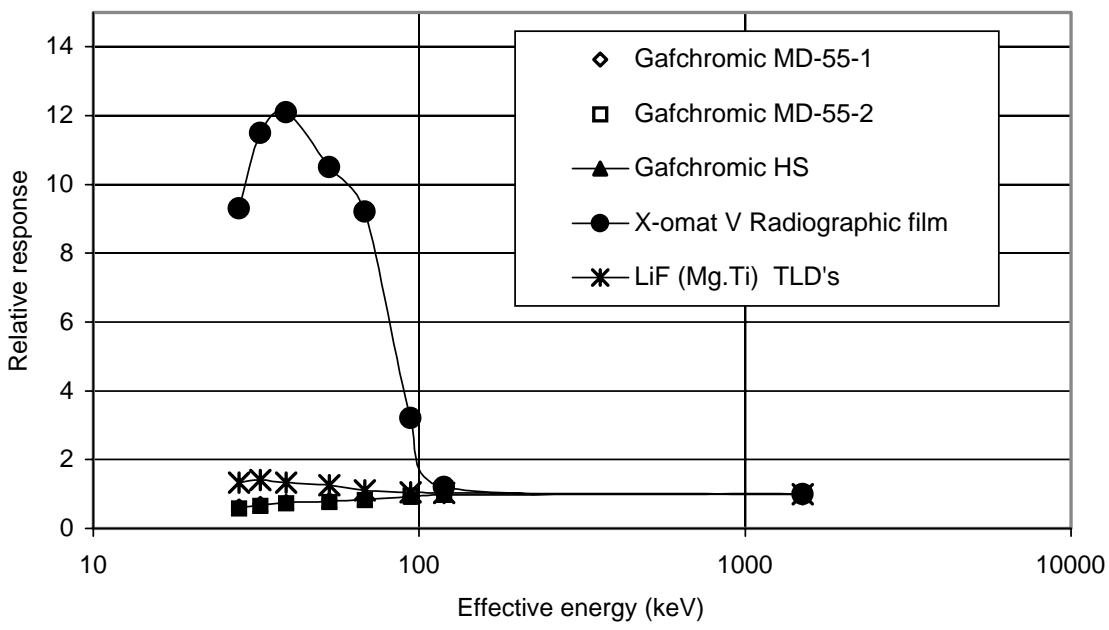

Fig. 24. Energy dependence of various medial radiation detectors in comparison to the radiochromic film. The radiochromic film is a relatively energy-independent radiation detector. 
energy response characteristics for radiochromic films compared to other two-dimensional radiation detectors.

\subsection{Dosimetry in water phantoms}

Dosimetry with radiochromic film products is a favorable and attractive alternative to radiographic films and other solid-state radiation detectors due to its properties such as energy dependence, physical toughness and automatic development. Many dosimetric evaluations need to be performed in liquid water environments for accurate calculations of doses in industrial and medical applications. Radiochromic films have various different construction types making their properties for water dosimetry vary depending on the type of the film. There are a few issues which may be raised when dealing with water dosimetry [118]. The main ones include: (a) the effects of water during exposure for the film dosimeter (neither TLDs or radiographic film are suitable for water dosimetry without some sort of protective coating placed over them); and (b) the response of the film due to the orientation with respect to the beam direction in the water. Many dosimeters show a directional dependence upon irradiation due to the variations on a microscopic scale of the radiation beam path before interaction at the point of measurement within the detector.

Fig. 25 shows the average visible penetration rate of water into the edges of the GAFCHROMIC ${ }^{\circledR}$ MD-55-2 film as a function of time. Water penetration into the edges of the film produces an opaque whitish colour and the values quoted in the figure are the visible points at which the film retained its original colour and OD value. The visible change recorded remains for an extended period of time and the film undergoes a permanent darkening of colour following the evaporation of the water absorbed. This process takes approximately 7-10 days but would be dependant on temperature and humidity values. There were no quoted variations in the water penetration rates due to temperature variations within the range of $15-30{ }^{\circ} \mathrm{C}$.

It was also noted that there was no measurable change in the optical density properties within the radiochromic MD-55-2 film due to the effects of water over a period of $24 \mathrm{~h}$. A slight change of

MD-55-2 water absorption

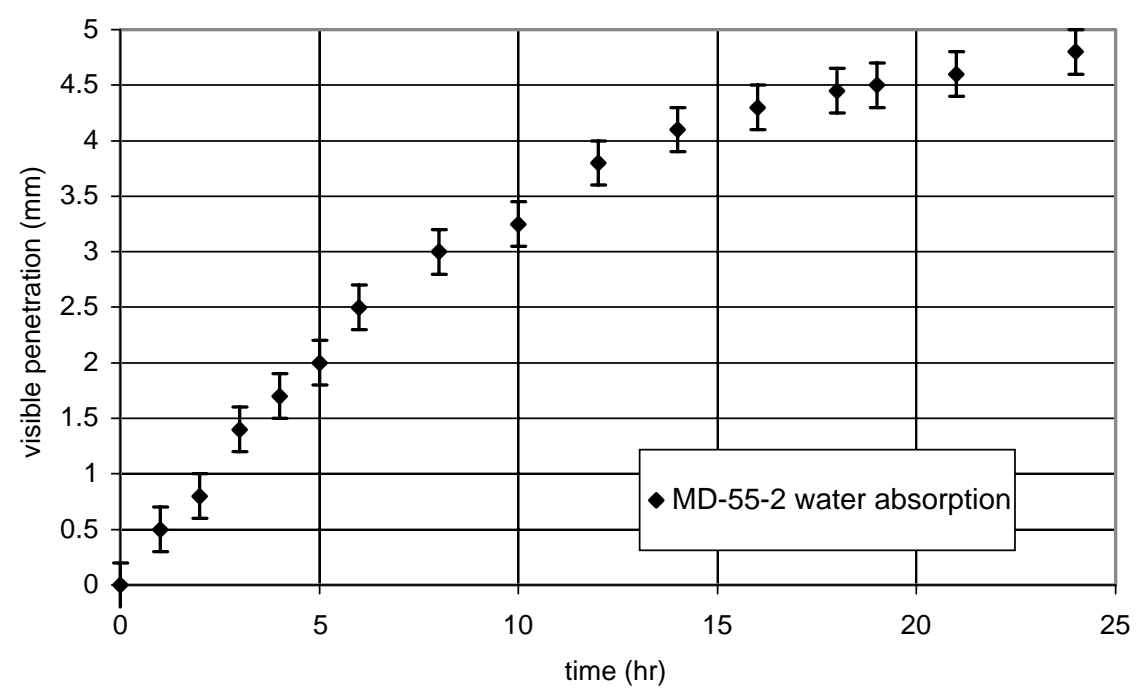

Fig. 25. Penetration rate of water leaking into a MD-55-2 radiochromic dosimeter. Radiation dosimetry is preferred in water due to the tissue equivalence of water and the ease of set-up. 
3-5\% was measured up to $2 \mathrm{~mm}$ inside the visible to eye water penetration mark. However, negligible variations were seen beyond this point. These results show that the MD-55-2 film is not significantly affected by placement in water during experimental exposure when the time frame is less than $24 \mathrm{~h}$, which is the case for most applications.

\subsection{Orientation of exposure effects}

Variations in measured radiation doses can occur due to the orientation of the film placed in the radiation field and the isotropic nature of the radiation in question. In medical applications, X-ray depth dose fields are mapped along the central axis of the radiation beam used. This tracks the beam path into a patient for treatment of a cancerous tumour at depth. There are problems associated with film exposure when the angle of incidence of the radiation field to the beam changes. This has been particularly noted when a film detector is placed parallel to the beam path. This has been attributed partially to scattering effects within the film structure and partially to the deformation in the measuring media caused by the placement of the film $[119,120]$. Radiochromic film, due to its relatively low atomic number and low density, does not suffer from a major influence from scattering effects. This is shown by its relatively independent response in a water phantom as a function of angle, which is highlighted in Fig. 26. In a solid phantom where the introduction of the film can cause air gaps to exist, a larger effect is seen and is also shown in Fig. 26. The size of this effect is proportional to the size of the air gap created by the introduction of the film into the media.

\subsection{Heat treatment processes for radiochromic films}

Due to the post-irradiation coloration characteristics of radiochromic film products, methods have been developed to "catalyse" the coloration process. This is performed with the use of a postirradiation heat treatment [121], whereby the chemical reactions occurring within the active structure of the film are enhanced. This can be performed easily with a dedicated controlled oven, which

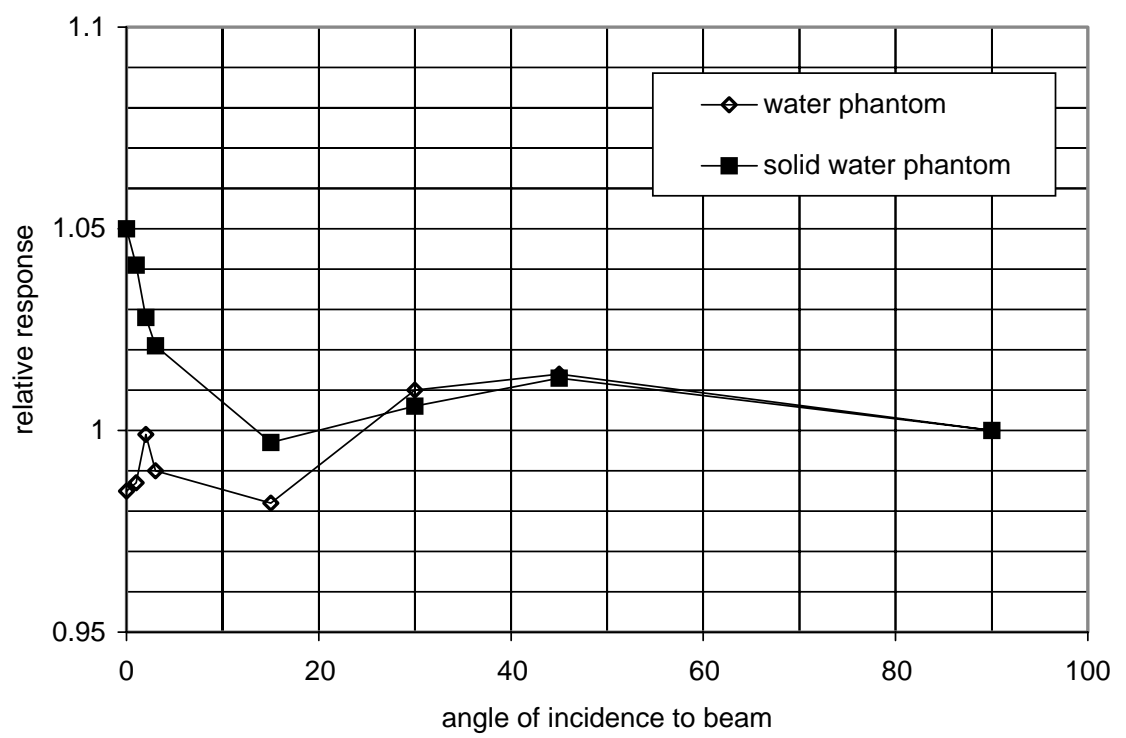

Fig. 26. Exposure effects of radiochromic film for perpendicular and parallel exposure to radiation highlighting the effects of parallel exposure of film products in solid phantom materials. 
possesses the correct temperature characteristics to match the desired outcome. Important issues arising with heat treatment of radiochromic film dosimeters are the reproducibility of the heating process, the uniformity of the heating process and the environmental effects associated with typical ovens used. Post-irradiation heat treatment of radiochromic film products is a well-established and recommended process. The amount of heating and temperature of optimal response is dependant on the product in use. An example is the GEX B3WINdose radiochromic film dosimeter which has a recommended heat treatment regimen of $5 \mathrm{~min}$ at $60{ }^{\circ} \mathrm{C}$ from heated air. If dosimeters are still in packaging, a longer time is required due to the extra material which needs to be heated before obtaining thermal equilibrium. Other recommendations to maintain a high level of accuracy include: (a) maintaining a thermal equilibrium within the heating oven at all time (i.e. leaving on to maintain temperature); (b) minimising the door open time for the system to reduce heat loss and thermal disequilibrium; (c) minimising thermal heat sinks around detectors such as packaging or material in contact with the films; and (d) loading films in a single layer within the oven to produce a better thermal equilibrium. Testing has been carried out by GEX Inc. on their dosimeters and various isolated effects have been found to be caused by temperature variations associated with opening the oven door, the heating process for a single layer of dosimeters in different positions within an oven and a loose bundle of dosimeters. Results showed that many factors influence the heating process, some of which are controllable and some of which are not. Fig. 27 shows a typical variation in temperature recorded when an oven door is opened to check or place dosimeters inside causing a fluctuation in the controlled temperature. The oven used here was a temperature-controlled TLD oven set to $65^{\circ} \mathrm{C}$ with the door opened for set time lengths. The results showed that a significant time interval was required to ramp back up to the appropriate temperature.

\subsection{Temperature and humidity}

Temperature, humidity and time effects of radiochromic film dosimeters were studied by Miller [122] using Far West Technology FWT-60-00 (batch no. 3E2) and Risø Labs B3 (batch no. 343510) radiochromic films in terms of the dose response.

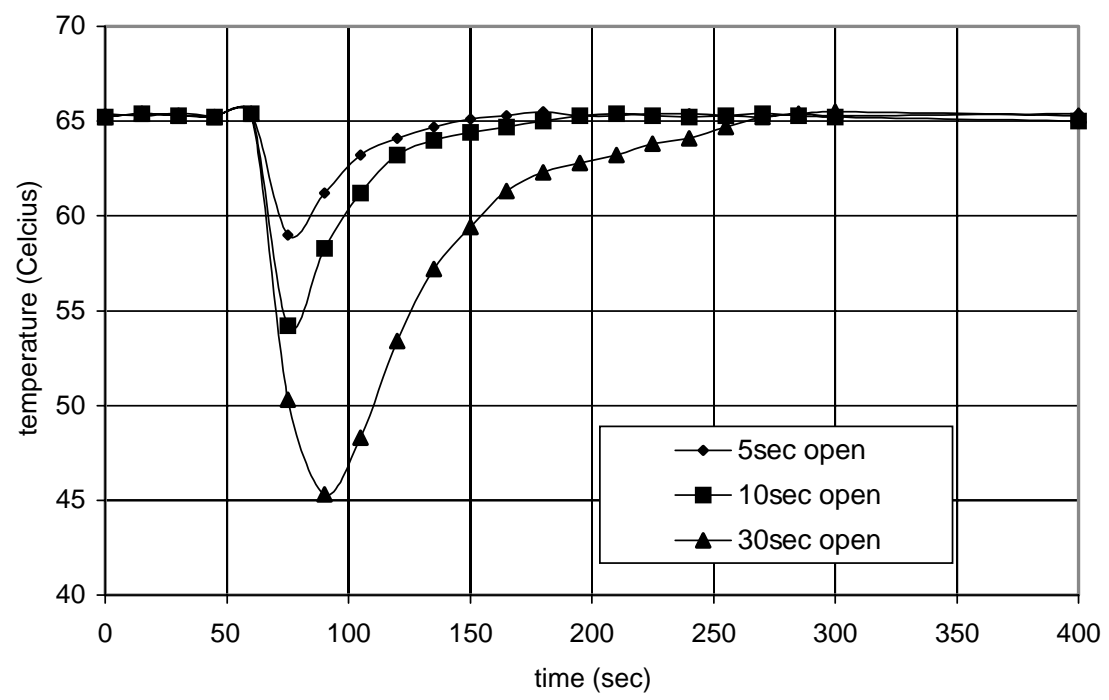

Fig. 27. Variation in temperature control within a heat treatment oven used for radiochromic film post-irradiation colouration stabilisation. 
During testing, both film types were kept in a number of relative humidity by suspending the film in saturated salt solutions for at least $24 \mathrm{~h}(48 \mathrm{~h}$ in the case of the electron irradiations) prior to irradiation so that each temperature at irradiation had 12 different films ( 6 for each film type) at 6 different relative humidity. Irradiation was done using ${ }^{60} \mathrm{Co} \gamma$-rays and $10 \mathrm{MeV}$ electrons. After irradiation, the films were heated to $60{ }^{\circ} \mathrm{C}$ for $5 \mathrm{~min}$ and the absorption then measured at 605 and $510 \mathrm{~nm}$.

It was found that for the $\gamma$-irradiation of the FWT-60-00, there was a strong increase in the response at $510 \mathrm{~nm}$ for very high relative humidity (94\%) and high temperatures $\left(50-60{ }^{\circ} \mathrm{C}\right)$, caused by the turbidity of the dosimeter. Since there was a simultaneous decrease in response at $605 \mathrm{~nm}$, the results are invalidated at both 510 and $605 \mathrm{~nm}$ for high relative humidity. The turbidity seen at high temperatures may also affect the dose response of the FWT film at lower temperatures. The variation in response for this specific batch and irradiation conditions for the $20-55 \%$ relative humidity range was $2.5 \pm 1 \%$ per $10{ }^{\circ} \mathrm{C}$.

For the $\gamma$-irradiation of the Risø Labs B3 film, there was no visible sign of turbidity at high relative humidity and temperatures. However, there was still a "significantly different response variation". The variation of the response for the batch and irradiation conditions of the B3 film was found to be $5 \pm 1 \%$ per $10{ }^{\circ} \mathrm{C}$.

When both the FWT and B3 films were irradiated with electrons, the dose responses were found to be similar to those of the $\gamma$-irradiation. However, since the two irradiations were not made at identical temperatures, this may account for some of the differences.

The response stability of the dosimeters was studied by irradiating both types to $25 \mathrm{kGy}$ and storing them in different conditions. The FWT-60-00 dosimeters were stored under two different conditions: (a) $22{ }^{\circ} \mathrm{C}$ and $54 \%$ relative humidity to represent typical laboratory conditions; and (b) $60{ }^{\circ} \mathrm{C}$ and no relative humidity control to represent accelerated ageing. The variation of response with time for both storage conditions was approximately equal. However, there was an increase in response at lower humidity for the first few hours up to $300 \mathrm{~h}$ for (a) while the higher storage temperature caused a decrease in response for the first $100 \mathrm{~h}$ followed by an increased response at $+300 \mathrm{~h}$ for $(\mathrm{b})$.

The B3 dosimeters were stored under three different conditions: (a) $22{ }^{\circ} \mathrm{C}$ and at the same relative humidity used during irradiation; (b) heated for $5 \mathrm{~min}$ at $60{ }^{\circ} \mathrm{C}$ and then stored in similar conditions to (a); and (c) $60{ }^{\circ} \mathrm{C}$ with no relative humidity control. For (a) there was development of color at low temperatures while at other temperatures the response was stable for more than $100 \mathrm{~h}$, and for (c) there was a similar color development at low temperatures but a decrease in response after around $100 \mathrm{~h}$.

Both dosimeter types show relatively small dependence on temperature and relative humidity in the ranges of $20-40{ }^{\circ} \mathrm{C}$ and $25-50 \%$ relative humidity, with a variation in response of up to 30 or $40 \%$ over the full range. Sealing the dosimeter under controlled conditions in vapor-tight pouches may limit this variation in response. Similar results were found by McLaughlin et al. [40] who studied the dependence of radiochromic films on temperature and relative humidity. They studied the FWT-60-00 and FWT-460 radiochromic films, both being irradiated using ${ }^{60} \mathrm{Co}$ and $10 \mathrm{MeV}$ electrons to dosages of $7,15,30$ and $50 \mathrm{kGy}$ at four different relative humidity and four different irradiation temperatures. The $\gamma$-irradiation of the FWT-60-00 showed negligible response variation at $22-33{ }^{\circ} \mathrm{C}$ but a pronounced variation at higher temperatures and humidity. The FWT-460 dosimeter showed the same negligible response to $\gamma$-rays at lower temperatures but the variation at higher temperatures and humidity was not as pronounced as for the FWT-60-00. The dose response of the films irradiated with electrons was found to be similar to that of the $\gamma$-irradiation at relatively low doses but with a difference in response to increasing relative humidity at higher doses. 


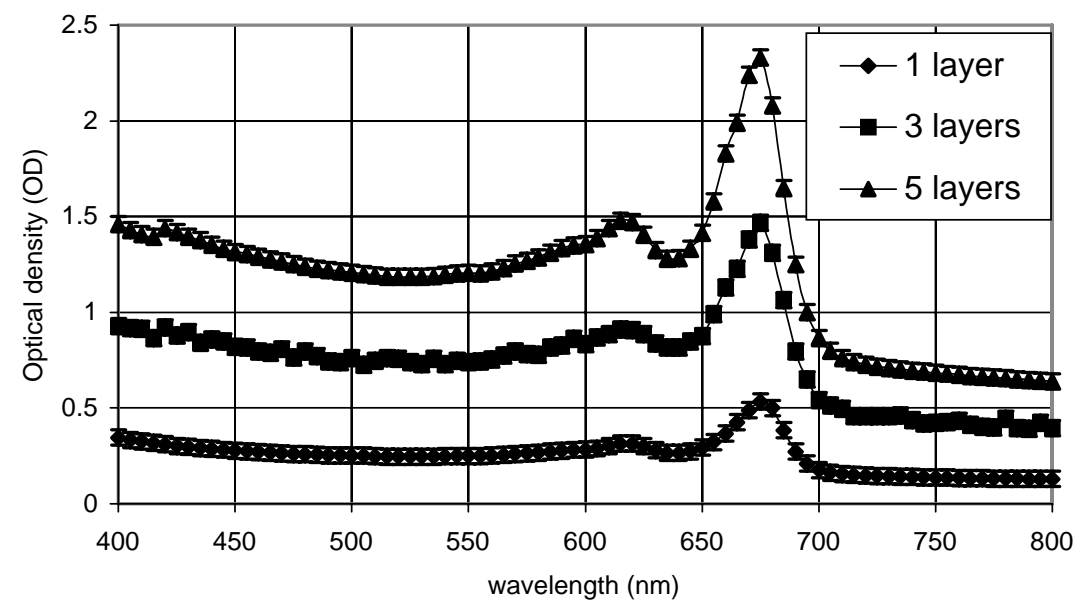

Fig. 28. Increases in dose sensitivity by layering of radiochromic film products together to produce a compounding optical density effect. Results are shown over the wavebands of $400-800 \mathrm{~nm}$.

\subsection{Increasing radiochromic dosimetry sensitivity}

Radiochromic film has a relatively low dose response compared to radiographic film such as Kodak ERD-2 film, which is used in applications such as intensity modulated radiotherapy dose verification. With the use of radiochromic film layers, a high-dose response detector, which is relatively energy independent, can be created [102]. Using the principle described by the BeerLambert law that the light absorbed by a medium varies exponentially with the path length of the light in the medium, one can effectively increase the change in optical density with the absorbed dose by layering films together. Depending on the effective position of measurement requirements and the flexibility of the dosimeter required, any amount of layers can be added together for a relatively linear increase in sensitivity with radiochromic films. This is demonstrated in Fig. 28

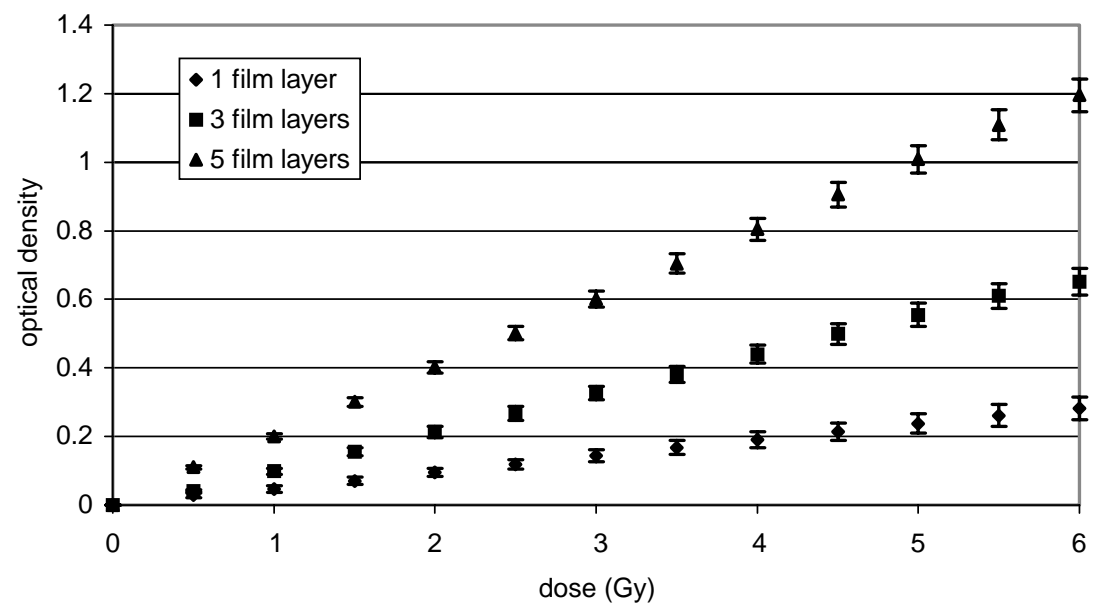

Fig. 29. Increased sensitivity achieved at the specific wavelength of $660 \mathrm{~nm}$ for three and five film layers. The increase required can be matched by the number of layers used. 


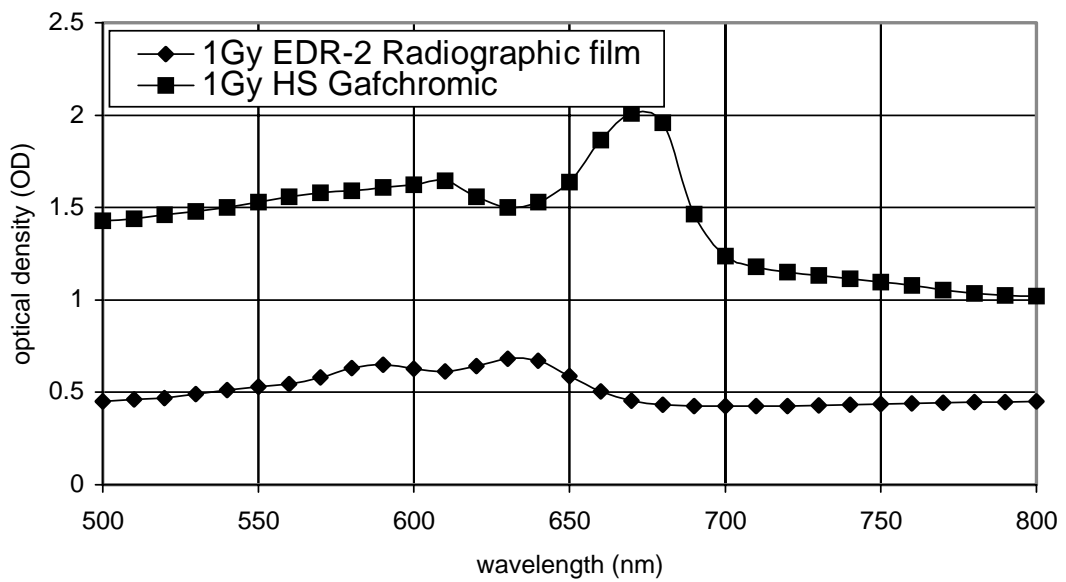

Fig. 30. Optical density absorption for high sensitivity GAFCHROMIC ${ }^{\mathbb{R}}$ HS film compared to Kodak EDR-2 radiographic film which is commonly used for imaging and dosimetry in radiotherapy applications.

which shows the increase in the optical density associated with layering radiochromic films together, which have all received the same absorbed dose.

At a specific wavelength $(660 \mathrm{~nm})$ the increase in sensitivity is shown in Fig. 29.

Fig. 30 shows the gross optical density over the spectral wavelengths of 500-800 nm for a EDR2 radiographic film and five layers of GAFCHROMIC ${ }^{\mathbb{R}}$ HS films, which were both irradiated to a dose of 1 Gy. The gross optical density results for the five layers of GAFCHROMIC ${ }^{\circledR}$ HS films are higher than the EDR-2 radiographic film for most wavelengths with the absorption peak located at approximately $675 \mathrm{~nm}$. For the EDR-2 film, the gross optical density is lower for an irradiation of 1 Gy with smaller peaks located at approximately 580 and $630 \mathrm{~nm}$.

Comparisons of the net optical densities show the achievable sensitivity increase (with five layers of GAFCHROMIC ${ }^{\circledR}$ HS films) when compared to the EDR-2 radiographic film in the wavelength range of 600-750 nm. The results shown in Fig. 31 are the net OD for absorbed doses of 100 and $200 \mathrm{cGy}$. As can be observed, the five layers of HS films produce a larger response to the dose within the wavelength range of approximately $665-685 \mathrm{~nm}$, which corresponds to the major peak.

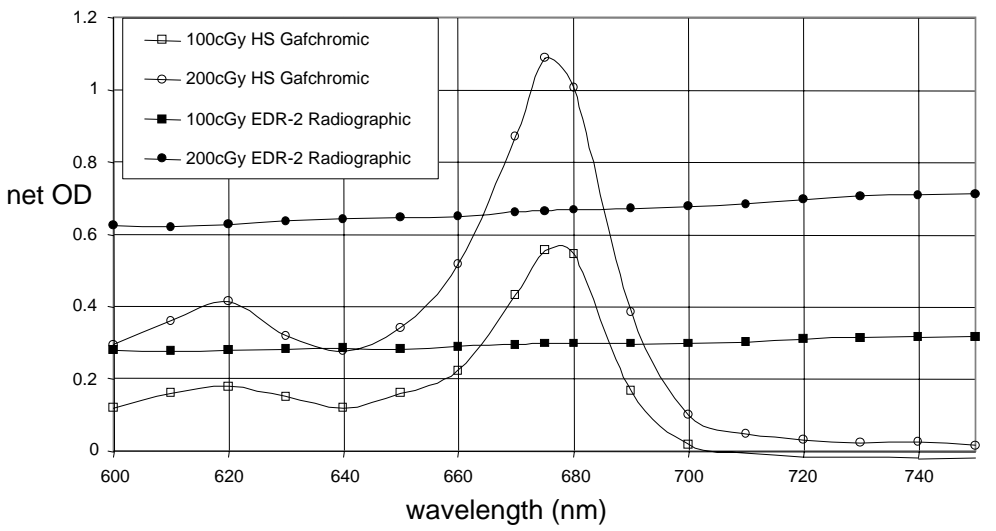

Fig. 31. Net dose response of the layered radiochromic film and radiographic film showing that a greater sensitivity is achievable at specific wavelengths for radiochromic film compared to radiographic film. 


\section{Densitometry systems: evaluation of radiochromic film dose}

The term "dosimetry" refers to some form of quantification. If only a qualitative picture of radiation interactions was required, the constraints placed on the digitisation of the optical density at a given point on a film would not need to be so rigorous. However, for film dosimetry as a quantitative process, it is ideal to convert the analogue film information into digital data for the ultimate quantification of dose accurately and reproducibly. A digitised film can provide a twodimensional data set describing the film coloration, which has resulted from the dose deposited in that plane. Although theoretically the ultimate limit of resolution is the dimension of each "activated" molecule on the film, practically the spatial resolution of the data set is limited by the sampling rate set in the scanner/densitometer software. Sometimes it is more useful to sample at a rate which is consistent with the data required for a specific patient treatment and the spatial resolution of the planning software used to calculate the patient absorbed dose during therapy. In this way, the planning data set and the dosimetry data set can be superimposed and a comparison made between the expected and actual dose.

To maximize the confidence in the measured dose, it is necessary to have a good relationship between the pixel value and the dose, i.e. a correct change in the pixel value for the given required doses. Choosing films with a suitable characteristic curve (i.e. dose response) or by exposing the film appropriately (to the most effective dose) can achieve this, so that the pixel values to be measured are in the linear portion of the dose response curve. The correct bit level of data acquisition is also required for the level of accuracy needed. An 8-bit software analysis tool (256 resolution) would be inadequate if it does not allow the level of accuracy required to be seen. By using 256-bits of information, a 1 pixel change is approximately $0.5 \%$. This may not be adequate. As such, normally scientists use 12- or 16-bit scanning resolution for film analyses or use the direct analogue signal for data processing.

\subsection{Densitometer types}

Many densitometers have been built exclusively for radiation therapy purposes. Densitometry is not however limited to these devices. Highly accurate photo-spectrometers have been used routinely as well as numerous office 'document' scanners with varying degrees of accuracy. Due to the inexpensive nature and the availability of office scanners, these devices have been used quite extensively for researchers and scientists who do not have access to larger and more expensive equipment. Fortunately, some of these document scanners appear not only to be able to operate automatically, providing a high-quality image, but also permit the user to disable certain features in the software. The disabling of smoothing and/or sharpening image processing features is a desirable factor to ascertain raw data which can be manipulated later and would appear to make some of these scanners suitable for use in film dosimetry.

The process of analogue to digital conversion from a film image to a digital data set is not always a simple one, and is subjected to corruption or distortion. Should the film dosimetry data be corrupted, the resulting data set will be invalid for that exposure. However, if it is distorted, it may be difficult to identify the subtle but dosimetrically significant changes in the resulting data set. Consequently, it is important to identify a process whereby the analogue film darkness can be faithfully converted to digital data. A brief overview of currently used densitometry systems and the general characteristics of these devices in terms of radiochromic film evaluation efficiency are included. Film dosimeters require the ability to accurately measure the radiochromic film absorbed dose. In doing so, they must also have an adequate latitude (the ability to digitise a 
range of film optical densities, maintaining good resolution and avoiding saturation) and reproducibility.

There are two distinct processes in image capture for image analysis. The first is to use a point densitometer which can be either stationary or moving and the film which can be stationary or moving. Using an appropriate manual or computer control, the optical density of a specified position can be determined but only one point at a time. Using this type of system, variables in the quantitative analysis of film can include characteristics of the light source, spatial resolution of the movement, linearity of signal detection, characteristics of the detector system, positional accuracy, environmental effects on film, software implications (bit resolution) and acquisition time. The other method of densitometry is the use of multiple detection systems which can measure multiple points at a time such as scanners using charge-coupled device (CCD) technology. Similar considerations are required as above with one main concern being the pixel size for the given device. Some of the characteristics which can affect the quantitative analysis of radiochromic film will be explained in summary below.

\subsection{Light source effects}

The presence of a light source for the analysis of radiochromic film products produces many variables which can affect the accuracy of the calculated absolute dose [84,98,123-125]. The largest consideration for radiochromic film is the wavelength of the light source. As seen in Section 2, a complex visible light absorption spectrum is observed with radiochromic film materials. The use of different wavelengths for analyses can produce outputs which can vary in the vicinity of the order of 1 magnitude (more than 10 times difference). Thus, the emission spectrum details of the employed densitometer are very important. Depending on the application, one might want to have the maximum sensitivity to dose exposure, which would mean that the waveband of the measurement light source should be matched to the absorption peak of the radiochromic film product. In the case of the MD-55-2 film, the peak is at $676 \mathrm{~nm}$ (red region). This could be achieved with the use of a photo-spectrometer [95] with analyses directly made at the wavelength of choice +1 to $2 \mathrm{~nm}$. Helium-neon laser scanners also have a specific wavelength of readout $(632.8 \mathrm{~nm})[83,126]$ which may or may not coincide with a high absorption point on the radiochromic film. In reality, most densitometers have a broader waveband and use devices such as light emitting diodes [85,127-130], laser diodes [131], or fluorescent light sources [56,57,66,85,132-136]. Sometimes band pass filters are used to convert a broad-band light source to a more sensitive or usable range [137]. Laser diodes can have slightly broad wavebands (of the order of 10-20 nm) and be optimised to specific wavelengths. LEDs tend to have even broader wavebands $(20-50 \mathrm{~nm})$ and again can be tailored to specific regions within the visible spectrum. Fluorescent lights have sources which cover the entire visible range, but variations can occur due to the "type" of light wanted by the use of differing fluorescent materials within the tube. High-resolution scanners and document scanners normally employ fluorescent lights as they are the most practical ones in more conventional uses (analysing radiographic film or scanning colour pictures, etc.). An important point of consideration when using fluorescent light sources is that they often contain an ultraviolet component in their emission spectra, which could affect the post-irradiation optical density of the film. Other characteristics affecting the final results include the size, uniformity and intensity of the light source. Another important factor can also be the polarization of the light source. If a polarised light source and a polarised detection system are present, the phase shifting effects of the radiochromic film can significantly affect the measured output. The presence of interference fringes caused by reflections between the film and holding plates can also be of significance when analysing results at a specific wavelength [98]. 


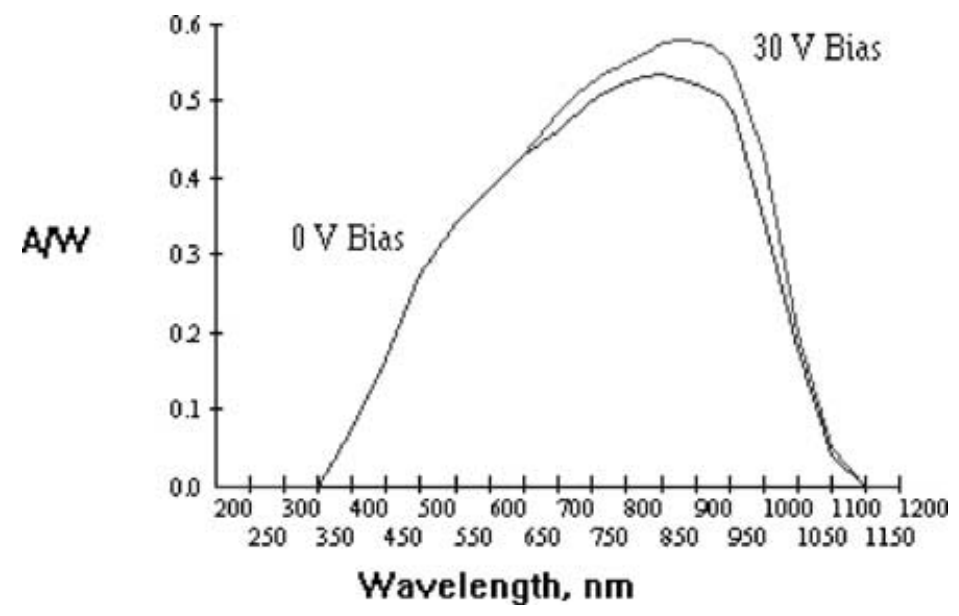

Fig. 32. Spectral response [photo sensitivity (A/W) vs. wavelength (nm)] of a common photodiode detector used in radiochromic film point densitometers.

\subsection{Transmitted light detectors}

The detection system used for analysis can also significantly affect the measured transmitted light and can vary due to characteristics such as linearity of output, signal resolution, sensitivity and spectral sensitivity. Low-sensitivity detectors normally employ solid-state devices, such as photodiodes, which are commonly used for point densitometers in translation-type scanners such as water tank densitometers. Document scanners and high-resolution densitometers mainly use charge-coupled devices (CCDs) which can produce a high sensitivity. The spectral sensitivity of a light detector can vary significantly, as shown in Fig. 32, which is a manufacturer's specification for a photon diode [138]. Changes in the spectral composition of the incident light as well as colour changes produced by the film itself can produce a quantitative effect on the measured optical density.

The signal resolution of a detector can significantly affect the desired accuracy of the measurements made. In conjunction with the transmitted intensity, this parameter controls the achievable level of resolution in the output. Intensity resolution is normally not a major problem for devices which output analogue signals, as the signals are read by devices, such as a voltmeter, which will normally have a large scale range, providing adequate signal resolution. Devices that capture the image and change to a pixel value internally are limited by the resolution of the capturing circuit. An 8-bit resolution will only give 255 discrete steps of resolution, which may not provide a sufficiently high level of accuracy. In medical dosimetry, normally 12-bit (4096) or 16-bit $(65,536)$ steps are used to provide an adequate level of resolution for dose analyses on radiochromic films.

\subsection{Spatial resolution}

The spatial resolution of a densitometry system is defined by several factors. These include: (a) the minimum space between successive readings (i.e. the physical resolution), which is dependant on the pixel size of the image created and the size of the void area in between the active matrix array elements; (b) the light source size causing variations in the transmitted light due to image flaring; (c) scattering effects from the film and other elements within the reader field of view; and (d) other sources of stray/ambient light within the reader. The actual spatial resolution of a device is easily tested with a line pair pattern, thus the resolution is often quoted as line pair/mm. The resolution is the ability to define the full width half maximum (FWHM) positions of two parallel lines in close 
proximity of each other. When a spatial resolution is chosen for a given experiment, factors such as the theoretical calculation one is trying to match as well as the size of the film which will be created should be taken into account for ease of data processing. A level of resolution dramatically higher than the level actually required will slow down the entire calculation process.

\subsection{Environmental effects}

When a film is placed in a densitometer for analysis, it encounters a new set of parameters which may not necessarily be present in its normal conditions and which may vary considerably due to its position and time within the densitometer. One of the largest changeable factors here is the temperature of the film within the scanner during readout and processing. As a densitometer is an electrically powered device, a degree of heat is produced within. The actual temperature at the point of readout is often hard to determine but can vary considerably with the length of time the device has been on in the standby mode and how long the device has actually been measuring. Some radiochromic film products exhibit a change in the visible absorption spectra as a function of temperature. This effect can significantly affect the measured output or optical density if the scanner is set-up in a way to maximize output. Some film readers use a light source in the region of $670 \mathrm{~nm}$, which lies near the maximum absorption peak of the MD-55 films at room temperature. This peak, however, is temperature sensitive and moves with temperature. If the film is heated by the densitometer during readout, the visible absorption spectra of the film will shift, causing the transmitted light at the specific wavelength of $670 \mathrm{~nm}$ to change dramatically. The amount of ultraviolet light produced by the densitometer can also affect the measured optical density of the film due to extra darkening whilst in the reader. This can be especially important for fluorescent light densitometers which do possess a significant UV component in their emission spectrum.

\section{Product review}

\subsection{GEX B3 WINdose radiochromic film dosimeter [122,139-141]}

Manufacturer: GEX Corporation, 7330 S. Alton Way, 12-I Centenial, CO 80112, USA.

Physical properties. Dosimeters are supplied as $1.0 \mathrm{~cm}$ squares which are laminated with a $50 \mu \mathrm{m}$ thick paper overlay that has a $5.5 \mathrm{~mm}$ aperture. Each overlay is marked with a unique identifier for quality assurance purposes to assign individuality to each dosimeter. There is a variation in the physical thickness for these dosimeters of approximately $2 \%$ and the physical thickness is specified for each batch (nominally around $0.0183 \mathrm{~mm}$ ).

Performance characteristics. B3 WINdose radiochromic film dosimeters undergo a color change when exposed to ionising radiation. It has a colour absorption peak at approximately $554 \mathrm{~nm}$, which is the manufacturer's recommended wavelength for readout. There are no known dose-rate effects for B3 dosimeters at most medical and industrial dose rates. Dosimeters have an effective upper temperature of use of $60{ }^{\circ} \mathrm{C}$. B3 dosimeters have an effective shelf life of approximately 5 years. Dosimeter response is affected by temperature and humidity factors, both during and after irradiation and also the water content in the film during irradiation. Recommendations for use are to keep detectors in the sealed containers as long as possible before readout. That is, if possible, irradiate dosimeters in packaging. B3 dosimeters are sensitive to ultraviolet radiation and as such should be protected from UV sources (including sunlight and fluorescent light exposure) as much as possible. The practical range of $\mathrm{B} 3$ dosimeters is from $100 \mathrm{~Gy}$ up to $80 \mathrm{kGy}$ providing dosimetry in the high-dose range. The coloration of B3 dosimeters may continue to develop for a period of time after 
irradiation and should be evaluated on a batch-specific calibration at the user's institution. Postirradiation coloration can be completed by heat treating the dosimeters for not less than 15 min and no longer than $6 \mathrm{~h}$ in the temperature range of $60-65^{\circ} \mathrm{C}$. This provides a stable dosimeter response for up to 18 months after treatment when stored at room temperature. B3 dosimeters can become statically charged under certain conditions, which can cause particles to be drawn to the detectors surface: these particles can interfere with the optical readout accuracy. Precautions and careful wiping of the film may be necessary.

\subsection{Radiochromic FWT-60 radiochromic film [142]}

(FWT also manufactures the Opti-Chromic series of dosimeters that are useful for lower doses than the FWT-60 series. However, they are supplied in cylindrical rod format instead of film format.)

\subsubsection{Manufacturing process}

(1) Manufacturing the dye and its components. FWT manufactures its own dyes that are used in the dosimeters. This insures that the dye is of high purity and quality. The nylon matrix that holds the dye goes through several conditioning steps. Clear and blemish-free dosimeters are the result of the extra steps in the manufacturing procedures.

(2) Solvent casting large sheets of dosimeters. Dye/nylon/solvent solutions are evenly spread over extremely flat sheets of glass. The solvents evaporate, leaving a free standing film which is then peeled from the glass. The goal of casting the dosimeters is an even thickness, which is best achieved by casting the dosimeters in sheets.

(3) Drying and ageing the sheets. The sheets are not completely dry after peeling. They are hung in cabinets with a continuous airflow for 3 months to finish the drying/curing process.

(4) Cutting the sheets into proper dosimeter size. The sheets are then cut into the required size. They are usually cut into $1 \mathrm{~cm} \times 1 \mathrm{~cm}$ squares for the FWT-60 dosimeters.

(5) Inspecting and sorting each dosimeter. Each FWT-60 dosimeter is visually checked and sorted by thickness.

\subsubsection{Chemical composition of radiochromic film}

The FWT-60 dosimeters are composed of hexa(hydroxyethyl) pararosaniline nitrile. The matrix that holds the dye is nylon. The film has a density of approximately $1.15 \mathrm{~g} \mathrm{~cm}^{-3}$ and a composition (by mass) of $63.7 \% \mathrm{C}, 12.0 \% \mathrm{~N}, 9.5 \% \mathrm{H}$ and $14.8 \% \mathrm{O}$.

\subsubsection{Handling, storage and irradiation of dosimeters}

The dosimeters are strong soft nylon films. They can be handled by picking them up with one's fingers but this can be difficult because they are so thin. Picking them up this way will leave fingerprints, which can change the optical density readings and thus the exposure data. For these reasons, handling the dosimeters with tweezers is suggested.

In addition to changing colour from penetrating radiation, the dosimeters will also change color on exposure to UV light below $370 \mathrm{~nm}$. Most artificial lights contain some light in this region and will cause a colour change in the dosimeters exposed to the light for very long time. Sunlight, of course, contains a large quantity of UV photons. Even sunlight through a window will contain enough UV photons to quickly alter the color of a dosimeter. For this reason, a complete survey of all forms of light is recommended for the area where the dosimeters will be exposed. If the area uses fluorescent lights or has some daylight, the area will probably need filters. 
A simple test for UV exposure is to place several uncovered dosimeters of known optical densities for $8 \mathrm{~h}$ in the work area where the actual dosimeters will be used. If the density change with the exposure is greater than 0.005 , the area needs to be filtered. For critical measurements, we recommend always filtering all light sources, including lights from electronic equipment.

Filtering can consist of covering the fluorescent tubes with filter sleeves, covering windows with UV film, covering light fixtures with UV film and purchasing UV-free products. Filters are available for fluorescent tubes, incandescent lamps and for windows. All of these materials are designed to block UV light and will do an adequate job of protecting the dosimeters from exposure.

Exposing the dosimeters to visible light for prolonged periods (in the order of days-weeks) may cause a decrease in their sensitivities. This can occur with no changes in the background. For this reason, storing the dosimeters in the dark is recommended.

\subsubsection{Storage}

Recommendation is made to store the dosimeters at $35-55 \%$ relative humidity and $15-30{ }^{\circ} \mathrm{C}$. This will ensure a long shelf life, which will be shortened by higher temperatures or higher humidity. There is a natural colour development that takes place in the film over time and poor storage conditions will speed this up. Under optimum conditions, the dosimeters should have a storage life of 3-5 years. Low temperatures will retard the ageing process, but too low a temperature can cause problems with condensation. On the other hand, high temperatures will accelerate the ageing, which shows up as a higher initial OD. Prolonged storage at less than $10 \%$ relative humidity can cause a permanent change in the sensitivity. High humidity above $70 \%$ relative humidity can cause the films to look cloudy and will cause them to stick together. Exposure to above $90 \%$ relative humidity may cause a permanent change in the sensitivity.

\subsubsection{Conditioning}

For best dosimetry results, the film should be conditioned to a tight temperature and relative humidity range for $24 \mathrm{~h}$ prior to irradiation. We condition films at $47-53 \%$ relative humidity and

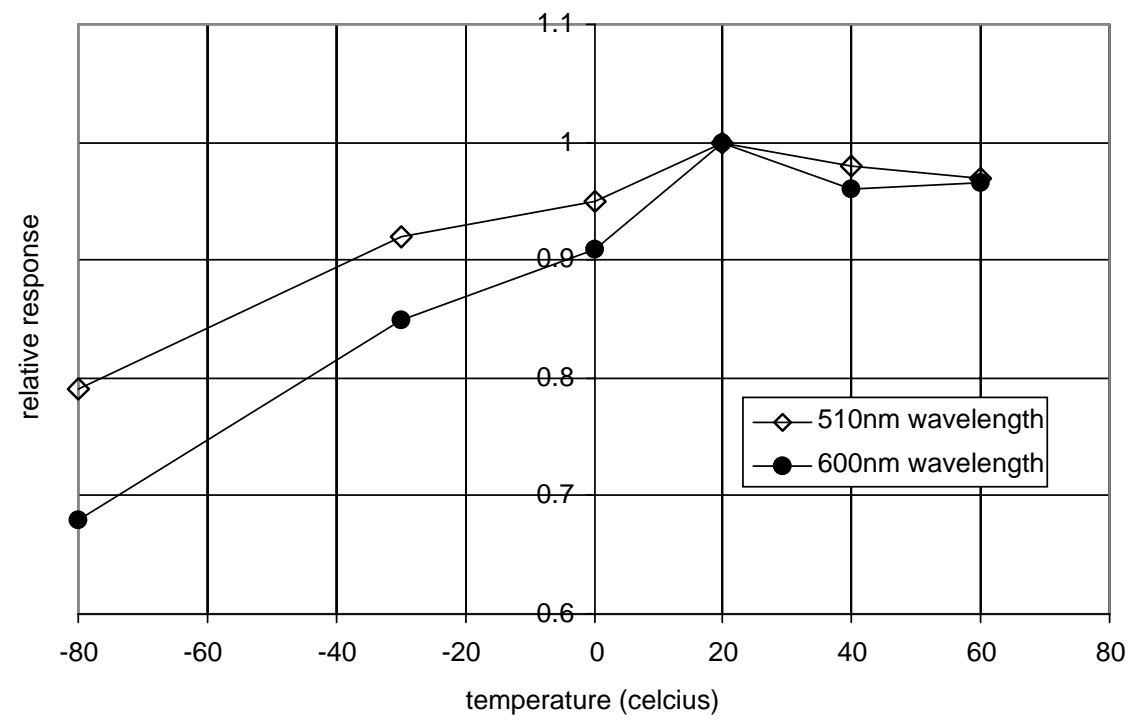

Fig. 33. Temperature dependence of FWT-60 radiochromic film during readout as measured at two specific wavelengths. Variations occur due to shifting absorption spectra as a function of temperature (results courtesy of Scot Larson, FWT Pty Ltd.). 


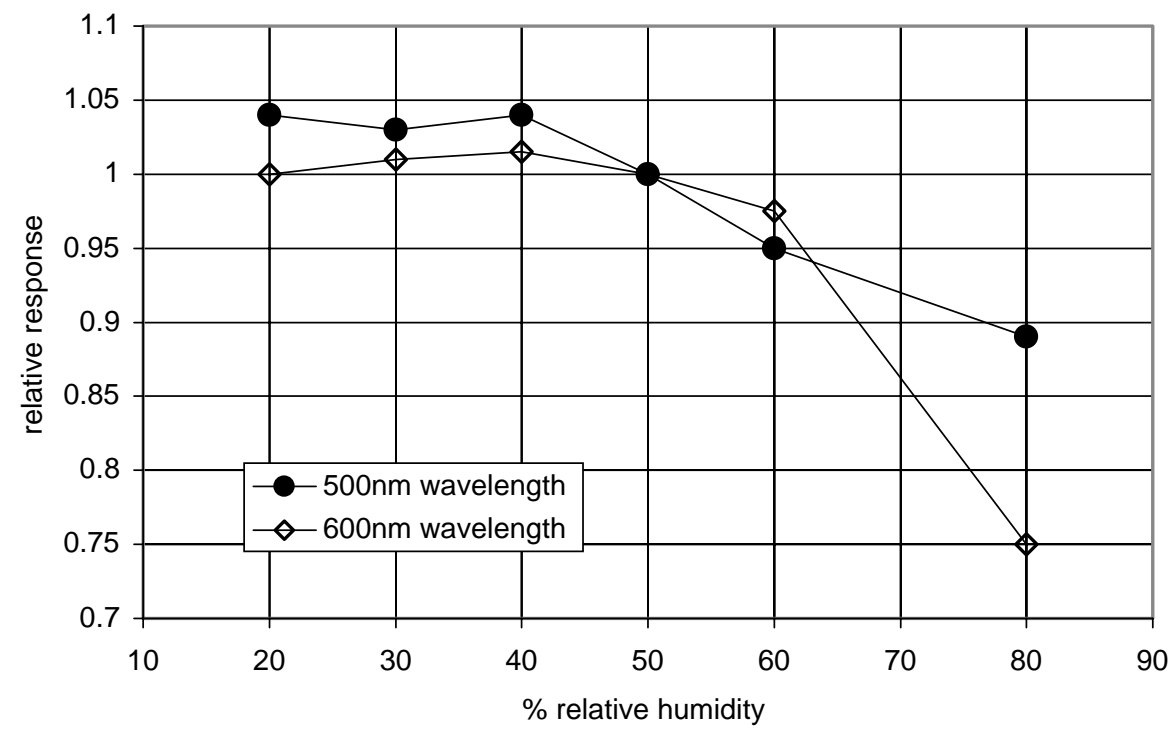

Fig. 34. Humidity dependence of FWT-60 radiochromic film during irradiation and measured at two specific wavelengths. A relatively high humidity produces a large effect on film response (results courtesy of Scot Larson, FWT Pty Ltd.).

20-21 ${ }^{\circ} \mathrm{C}$. Conditioning at a processing facility should be based on the calibration conditions and the ambient conditions.

\subsubsection{Temperature dependence}

The dosimeters have some temperature dependence. Fig. 33 shows a typical temperature response curve of the dosimeters. This curve is for a constant temperature during irradiation and will vary from batch to batch. Most dosimeters will be subjected to a varying temperature during irradiation.

\subsubsection{Humidity dependence}

The dosimeters have a humidity dependence. Fig. 34 shows a typical response of the dosimeters to variations in humidity. This curve will vary from batch to batch. For critical uses, the dosimeters can be placed in hermetic pouches to stabilize the humidity during irradiation.

\subsubsection{Colour development}

The dosimeters may take some time to develop full color. This time will vary depending on the humidity, exposure time and radiation energy. Typical times range from a few minutes to a few hours. At $24 \mathrm{~h}$, the entire color will be developed. The dosimeters can be easily tested for the local conditions by reading some test dosimeters over a period and noting the changes.

Generally speaking, the dosimeters will take longer to develop the color if the humidity is lower during irradiation. Higher dose rates will also delay the color change. With a longer irradiation time, the dosimeters seem to develop quicker. This is because the dosimeters were developing as they were being irradiated.

\subsubsection{Heat treatment}

The color change can be speeded up by heat treatment. The dosimeters are exposed to $90{ }^{\circ} \mathrm{C}$ for 2-3 min or $60{ }^{\circ} \mathrm{C}$ for 5-15 min for complete color development. 


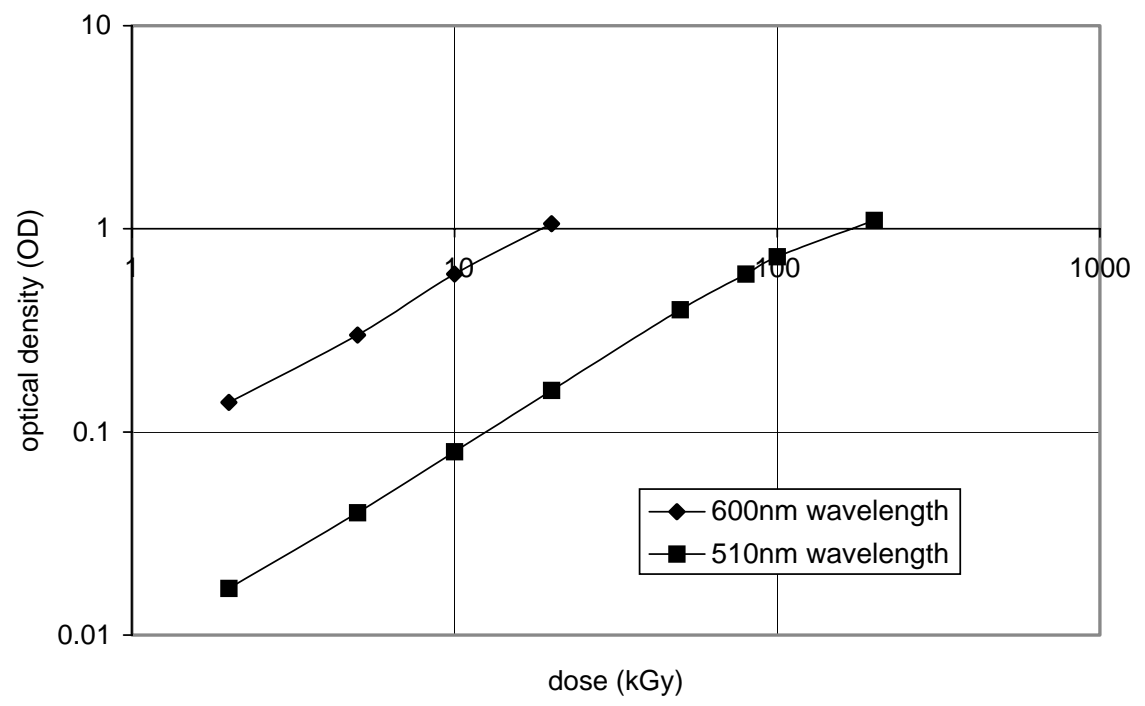

Fig. 35. Wavelength of readout response of FWT-60 radiochromic film detectors. A higher sensitivity is achieved at $600 \mathrm{~nm}$ due to an absorption peak located here (results courtesy of Scot Larson, FWT Pty Ltd.).

\subsubsection{Readout of dosimeters, wavelengths of interest}

The dosimeters have a peak wavelength for color change, which is centered on $605 \mathrm{~nm}$. The wavelengths to use for reading the films are 510 and 600 or $605 \mathrm{~nm}$. The latter two wavelengths are used for different dose ranges. Typical response curves are shown in Fig. 35 for the specified wavelength of readout. Variations will occur when different wavelengths are used for absorption analyses. Fig. 35 shows the dose response for the FWT-60 radiochromic film and Fig. 36 shows the dose response curve for the FWT-70 optichromic rods which are used for lower-dose delivery.

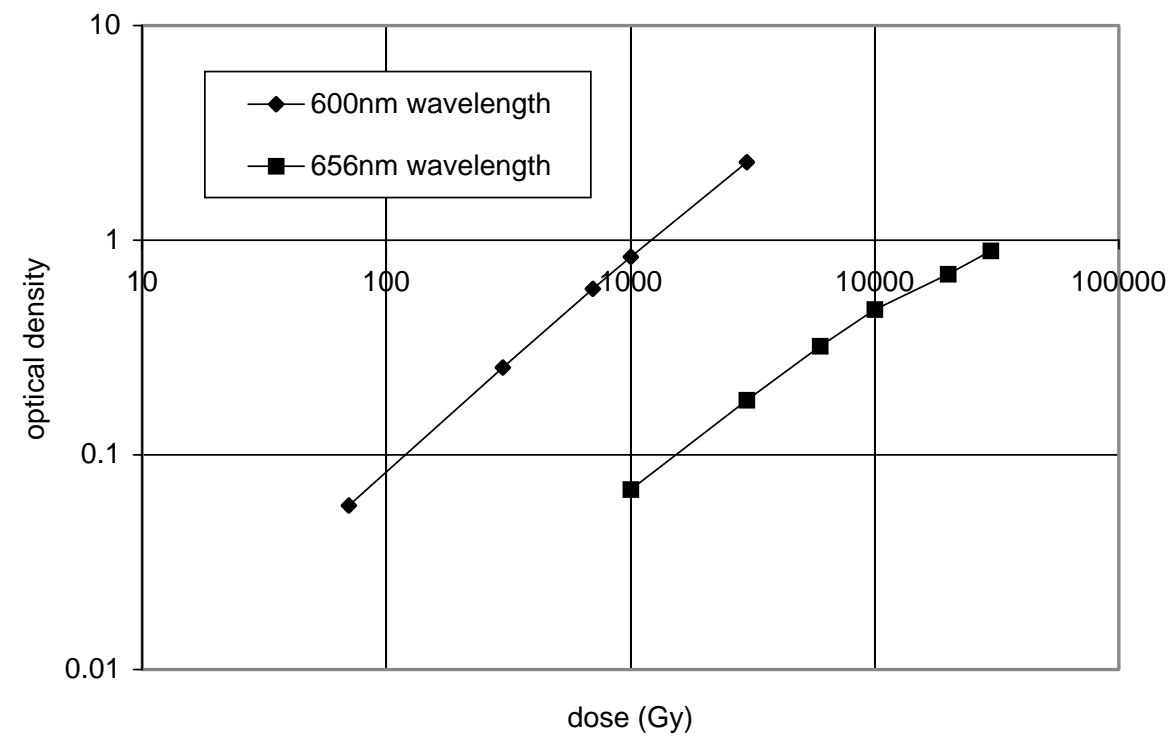

Fig. 36. Wavelength of readout response of FWT-70 radiochromic detectors, a higher sensitivity dosimeter in the FWT range. A higher sensitivity is achieved at $600 \mathrm{~nm}$ due to an absorption peak located here (results courtesy of Scot Larson, FWT Pty Ltd.). 
Table 5

GAFCHROMIC ${ }^{\circledR}$ HD-810 and D-200 films properties

\begin{tabular}{|c|c|c|}
\hline Property & GAFCHROMIC $^{\mathbb{R}}$ HD-810 film & GAFCHROMIC $^{\mathbb{R}}$ D-200 dosimeters \\
\hline Configuration & $\begin{array}{l}\text { Active layer and surface layer } \\
\text { on polyester substrate }\end{array}$ & $\begin{array}{l}\text { Active layer and surface layer } \\
\text { on polyester substrate }\end{array}$ \\
\hline Size & $\begin{array}{l}8 \text { in. } \times 10 \text { in. minimum; } \\
\text { other sizes upon request }\end{array}$ & $\begin{array}{l}1.2 \mathrm{~cm} \times 6 \mathrm{~cm} \text { paper holders with } 0.8 \mathrm{~cm} \\
\times 2.2 \mathrm{~cm} \text { window; other sizes upon request }\end{array}$ \\
\hline Substrates & 380 gauge clear transparent polyester & 380 gauge clear transparent polyester \\
\hline Active layer thicknesses & Nominally $6.5 \mu \mathrm{m}$ & Nominally $6.5 \mu \mathrm{m}$ \\
\hline Surface layer & Nominally $0.75 \mu \mathrm{m}$ & Nominally $0.75 \mu \mathrm{m}$ \\
\hline Sensitometric response & $\begin{array}{l}\text { Net density of } 0.30 \text { at } 100 \mathrm{~Gy} \\
\text { and } 1.15 \text { at } 500 \mathrm{~Gy}\end{array}$ & $\begin{array}{l}\text { Net density of } 0.30 \text { at } 100 \mathrm{~Gy} \\
\text { and } 1.15 \text { at } 500 \mathrm{~Gy}\end{array}$ \\
\hline Energy dependency & $\begin{array}{l}<5 \% \text { difference in net density for } \\
250 \mathrm{~Gy} \text { exposures at } 1 \text { and } 18 \mathrm{MeV}\end{array}$ & $\begin{array}{l}<5 \% \text { difference in net density for } \\
250 \text { Gy exposures at } 1 \text { and } 18 \mathrm{MeV}\end{array}$ \\
\hline $\begin{array}{l}\text { Dose fractionation } \\
\text { response }\end{array}$ & $\begin{array}{l}<5 \% \text { difference in net density for a } \\
\text { single } 40 \text { Gy dose and five cumulative } \\
8 \text { Gy doses at } 30 \text { min intervals }\end{array}$ & $\begin{array}{l}<5 \% \text { difference in net density for a single } \\
40 \text { Gy dose and five cumulative } 8 \text { Gy doses } \\
\text { at } 30 \text { min intervals }\end{array}$ \\
\hline Dose rate response & $\begin{array}{l}<5 \% \text { difference in net density for } 10 \mathrm{~Gy} \\
\text { exposures at rates of } 3.4 \text { and } 0.034 \mathrm{~Gy} \mathrm{~min}\end{array}$ & $\begin{array}{l}<5 \% \text { difference in net density for } 10 \mathrm{~Gy} \\
\text { exposures at rates of } 3.4 \text { and } 0.034 \mathrm{~Gy} \mathrm{~min}\end{array}$ \\
\hline Stability in light & $<0.005$ change in density per $1000 \mathrm{~lx}$ day & $<0.005$ change in density per $1000 \mathrm{~lx}$ day \\
\hline $\begin{array}{l}\text { Stability in dark } \\
\text { (pre-exposure) }\end{array}$ & $\begin{array}{l}<0.5 \times 10^{-3} \text { density change per day at } 23^{\circ} \mathrm{C} ; \\
<0.2 \times 10^{-3} \text { density change per day } \\
\text { refrigerated }\end{array}$ & $\begin{array}{l}<0.5 \times 10^{-3} \text { density change per day at } 23^{\circ} \mathrm{C} \\
<0.2 \times 10^{-3} \text { density change per day } \\
\text { refrigerated }\end{array}$ \\
\hline Uniformity, single sheet & $<8 \%$ sensitometric response difference & $<8 \%$ sensitometric response difference \\
\hline $\begin{array}{l}\text { Sheet-to-sheet } \\
\text { uniformity }\end{array}$ & $\begin{array}{l}<5 \% \text { sensitometric response } \\
\text { difference from mean }\end{array}$ & $\begin{array}{l}<5 \% \text { sensitometric response } \\
\text { difference from mean }\end{array}$ \\
\hline $\begin{array}{l}\text { Batch-to-batch } \\
\text { uniformity }\end{array}$ & $\begin{array}{l}<10 \% \text { sensitometric response } \\
\text { difference from mean }\end{array}$ & $\begin{array}{l}<10 \% \text { sensitometric response } \\
\text { difference from mean }\end{array}$ \\
\hline $\begin{array}{l}\text { Post-exposure } \\
\text { density growth }\end{array}$ & $\begin{array}{l}<12 \% \text { from } 1 \text { h to } 1 \text { day after exposure; } \\
<4 \% \text { from } 1 \text { to } 4 \text { days after exposure }\end{array}$ & $\begin{array}{l}<12 \% \text { from } 1 \text { h to } 1 \text { day after exposure; } \\
<4 \% \text { from } 1 \text { to } 4 \text { days after exposure }\end{array}$ \\
\hline
\end{tabular}

\subsection{ISP GAFCHROMIC ${ }^{\mathbb{R}}$ film products [143]}

ISP Technology is arguably the largest producer of radiochromic film products used in medical dosimetry due to their low dose range products which have uses in both therapeutic and diagnostic applications. Many figures shown throughout this text are representative of their medical dosimetry range. Brief information for their film ranges is given in tabular form (Tables 5 and 6). More detailed information is given for their relatively new product range of GAFCHROMIC ${ }^{\circledR}$ XR type R and XR type $\mathrm{T}$ films, which are higher sensitivity, diagnostic products.

\subsubsection{GAFCHROMIC ${ }^{\circledR}$ XR type $R$}

Fig. 37 shows the dose response of the GAFCHROMIC ${ }^{\circledR}$ XR type $\mathrm{R}$ dosimetry film. The densities were obtained with a Gretag-Macbeth D19C reflection densitometer using the cyan colour channel for measurements (i.e. analysis in the red region of the visible spectrum).

Fig. 38 shows the net density data from measurements of the response of the GAFCHROMIC ${ }^{\circledR}$ $\mathrm{XR}$ type $\mathrm{R}$ dosimetry film to X-rays generated at $60,80,100$ and $120 \mathrm{kVp}$. The response of the film to $80-120 \mathrm{kVp} \mathrm{X}$-rays is essentially energy independent, the net-density values for a given exposure 
Table 6

GAFCHROMIC ${ }^{\circledR}$ MD-55-2 and HS films properties

\begin{tabular}{|c|c|c|}
\hline Property & $\begin{array}{l}\text { GAFCHROMIC } \\
\text { radiochromic dosimetry film }\end{array}$ & $\begin{array}{l}\text { GAFCHROMIC }{ }^{\circledR} \text { HS } \\
\text { radiochromic dosimetry film }\end{array}$ \\
\hline Configuration & $\begin{array}{l}\text { Two active layers on polyester } \\
\text { substrates laminated with adhesive tape }\end{array}$ & $\begin{array}{l}\text { Active layer between polyester } \\
\text { substrates }\end{array}$ \\
\hline Size & $\begin{array}{l}5 \text { in. } \times 5 \text { in. minimum; } \\
\text { other sizes upon request }\end{array}$ & $\begin{array}{l}5 \text { in. } \times 5 \text { in. minimum; } \\
\text { other sizes upon request }\end{array}$ \\
\hline Substrates & 260 gauge clear transparent polyester & 380 gauge clear transparent polyester \\
\hline Active layer thicknesses & Nominally $2 \mu \mathrm{m} \times 16 \mu \mathrm{m}$ & Nominally $40 \mu \mathrm{m}$ \\
\hline Laminating tape & $\begin{array}{l}100 \text { gauge polyester with } \\
\text { double-sided adhesive layers } \\
\text { approximately } 1 \mu \mathrm{m} \text { thick }\end{array}$ & $-($ none $)$ \\
\hline Sensitometric response & $\begin{array}{l}\text { Net density of } 0.90 \text { at } 25 \mathrm{~Gy} \text { and } \\
1.75 \text { at } 50 \mathrm{~Gy}\end{array}$ & $\begin{array}{l}\text { Net density of } 0.80 \text { at } 10 \mathrm{~Gy} \text { and } \\
2.15 \text { at } 30 \mathrm{~Gy}\end{array}$ \\
\hline Energy dependency & $\begin{array}{l}<5 \% \text { difference in net density for } \\
50 \mathrm{~Gy} \text { exposures at } 1 \text { and } 18 \mathrm{MeV}\end{array}$ & $\begin{array}{l}<5 \% \text { difference in net density for } \\
20 \mathrm{~Gy} \text { exposures at } 1 \text { and } 18 \mathrm{MeV}\end{array}$ \\
\hline Dose fractionation response & $\begin{array}{l}<5 \% \text { difference in net density for a single } \\
40 \text { Gy dose and five cumulative } 8 \text { Gy } \\
\text { doses at } 30 \text { min intervals }\end{array}$ & $\begin{array}{l}<5 \% \text { difference in net density for a single } \\
30 \text { Gy dose and five cumulative } 6 \mathrm{~Gy} \\
\text { doses at } 30 \text { min intervals }\end{array}$ \\
\hline Dose rate response & $\begin{array}{l}<5 \% \text { difference in net density for } \\
10 \mathrm{~Gy} \text { exposures at rates of } 3.4 \\
\text { and } 0.034 \mathrm{~Gy} \mathrm{~min}\end{array}$ & 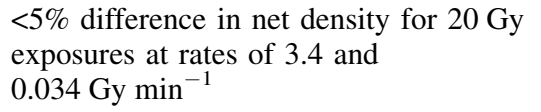 \\
\hline Stability in light & $<0.005$ change in density per 1000 lx day & $<0.01$ change in density per $10001 \mathrm{x}$ day \\
\hline $\begin{array}{l}\text { Stability in dark } \\
\text { (pre-exposure) }\end{array}$ & $\begin{array}{l}<0.5 \times 10^{-3} \text { density change per day at } \\
23{ }^{\circ} \mathrm{C} ;<0.2 \times 10^{-3} \text { density change } \\
\text { per day refrigerated }\end{array}$ & $\begin{array}{l}<0.5 \times 10^{-3} \text { density change per day at } \\
23{ }^{\circ} \mathrm{C} ;<0.2 \times 10^{-3} \text { density change } \\
\text { per day under refrigeration }\end{array}$ \\
\hline Uniformity, single sheet & $<8 \%$ sensitometric response difference & $<6 \%$ sensitometric response difference \\
\hline Sheet-to-sheet uniformity & $\begin{array}{l}<5 \% \text { sensitometric response difference } \\
\text { from mean }\end{array}$ & $\begin{array}{l}<5 \% \text { sensitometric response difference } \\
\text { from mean }\end{array}$ \\
\hline $\begin{array}{l}\text { Batch-to-batch } \\
\text { uniformity }\end{array}$ & $\begin{array}{l}<10 \% \text { sensitometric response difference } \\
\text { from mean }\end{array}$ & $\begin{array}{l}<10 \% \text { sensitometric response difference } \\
\text { from mean }\end{array}$ \\
\hline $\begin{array}{l}\text { Post-exposure } \\
\text { density growth }\end{array}$ & $\begin{array}{l}<12 \% \text { from } 1 \text { h to } 1 \text { day after exposure; } \\
<4 \% \text { from } 1 \text { to } 4 \text { days after exposure }\end{array}$ & $\begin{array}{l}<12 \% \text { from } 1 \text { h to } 1 \text { day after exposure; } \\
<2 \% \text { from } 1 \text { to } 4 \text { days after exposure }\end{array}$ \\
\hline
\end{tabular}

dose being within about $\pm 3 \%$. The response to $60 \mathrm{kVp} \mathrm{X}$-rays is, on average, about $7 \%$ lower than the response to $120 \mathrm{kVp} \mathrm{X}$-rays.

The GAFCHROMIC ${ }^{\mathbb{R}}$ XR type R dosimetry film experiences relatively no dose fractionation effects. It was also found that the response of the film was dose-rate independent over dose rates

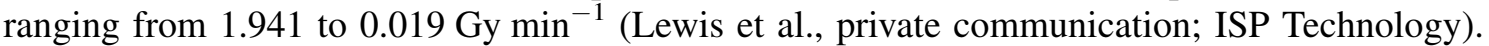

Fig. 39 shows the post-exposure density growth of the GAFCHROMIC ${ }^{\mathbb{R}}$ XR type $\mathrm{R}$ radiochromic dosimetry film from 1 to $24 \mathrm{~h}$ after exposure for various delivered doses and normalised to 1 at $24 \mathrm{~h}$. This reveals that post-exposure density growth, relative to the density at $24 \mathrm{~h}$, is essentially independent of the exposure dose, and is relatively proportional to the log of the time (Lewis et al., private communication). The density changes by about $8 \%$ between $1 \mathrm{~h}$ after exposure and $24 \mathrm{~h}$ after exposure, but the rate diminishes and the density changes by less than $2 \%$ over the next $72 \mathrm{~h}$.

Single sheet uniformity is quoted as $2 \%$ from manufacturer's data sheet. 


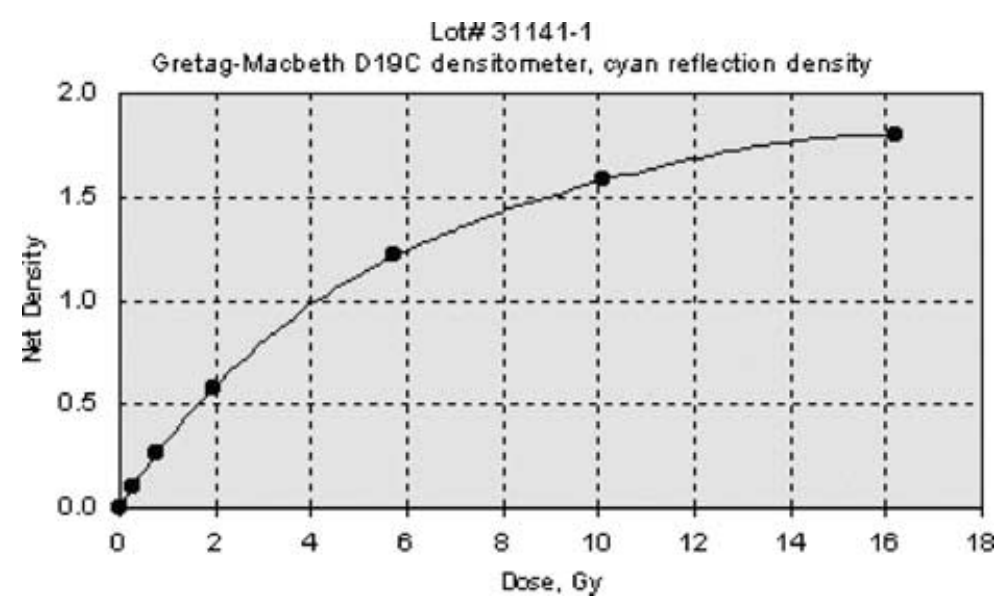

Fig. 37. Dose sensitivity of the GAFCHROMIC ${ }^{\circledR}$ XR type $\mathrm{R}$ radiochromic film (results courtesy of David Lewis, ISP Technology).

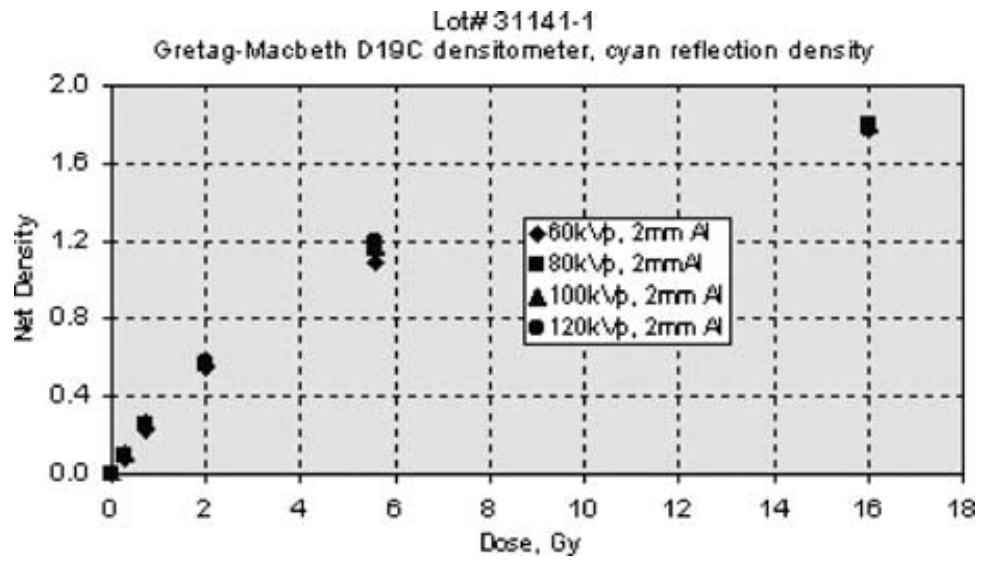

Fig. 38. Energy dependence of the GAFCHROMIC ${ }^{\circledR}$ XR type R radiochromic film in the range of $60-120 \mathrm{kVp}$ (results courtesy of David Lewis, ISP Technology).

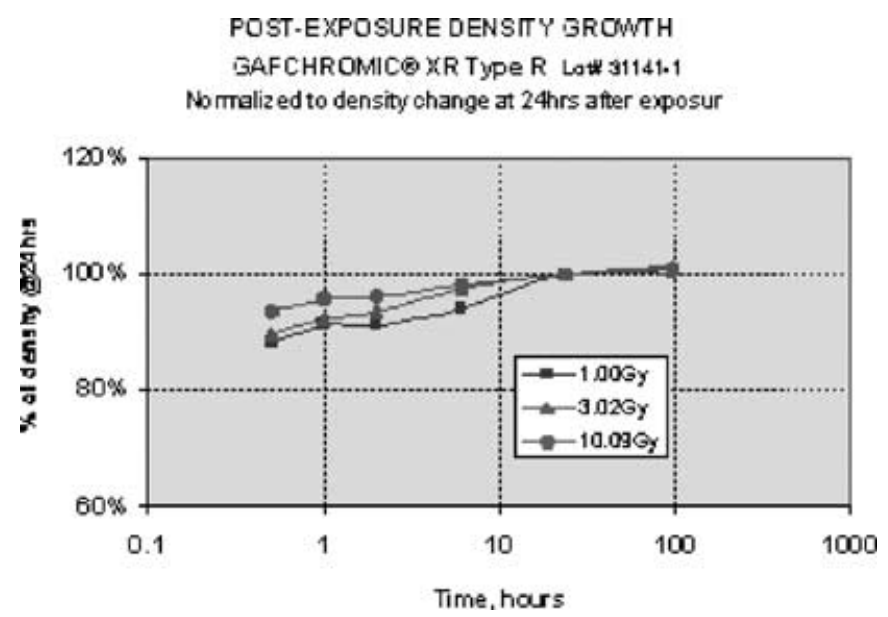

Fig. 39. Post-irradiation density growth for GAFCHROMIC ${ }^{\circledR}$ XR type R radiochromic film when exposed to various absorbed doses (results courtesy of David Lewis, ISP Technology). 


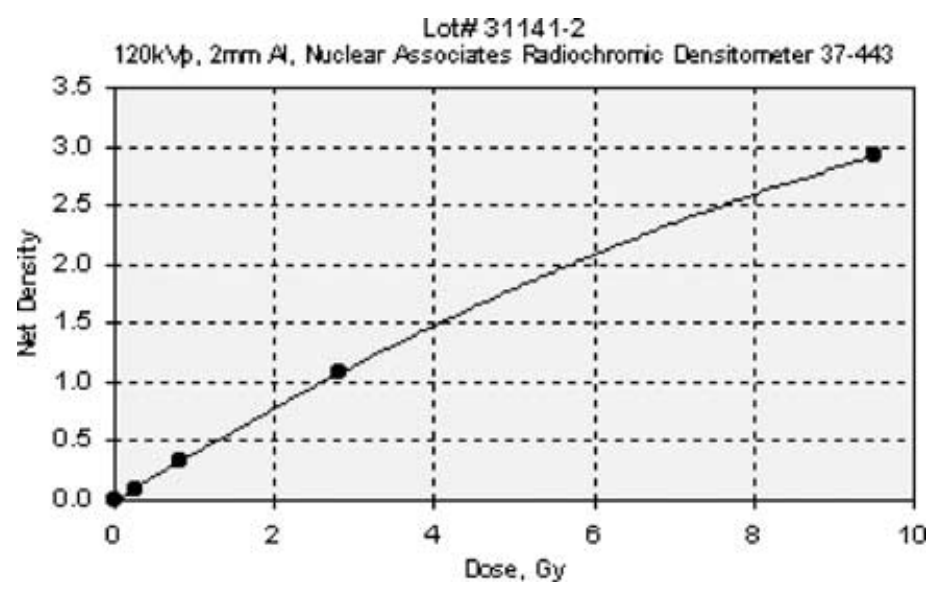

Fig. 40. Dose sensitivity of the GAFCHROMIC ${ }^{\circledR}$ XR type T radiochromic film for a lot number (results courtesy of David Lewis, ISP Technology).

\subsubsection{GAFCHROMIC ${ }^{\mathbb{R}}$ XR type T film}

Fig. 40 shows the dose response of the GAFCHROMIC ${ }^{\circledR}$ XR type $T$ radiochromic dosimetry film. The density measurements were made with a nuclear associates radiochromic densitometer, model 37-443. The net density is the density of the film sample after exposure minus the density of the film sample before exposure.

With this densitometer, the upper limit for exposure dose is about $10 \mathrm{~Gy}$ and the response is almost linear with the dose. This upper limit can be extended with the use of a densitometer with a broader waveband or a different wavelength which does not specifically lie near the visible absorption peak of this product.

Fig. 41 shows the energy response of the XR type T radiochromic dosimetry film with the use of 80,100 and $120 \mathrm{kVp}$ X-rays. Linear fits established for each of the three data sets have slopes that are within $0.4 \%$, demonstrating that the media is energy independent in this energy range.

Below $80 \mathrm{kVp}$, a larger energy response is observed and is shown in Fig. 42. Response to $60 \mathrm{kVp} \mathrm{X}$-rays is about $6 \%$ lower than the response to $80 \mathrm{kVp} \mathrm{X}$-rays. However, the response falls off more sharply at lower energies. When compared to the response to $60 \mathrm{kVp} \mathrm{X}$-rays, the response to $40 \mathrm{kVp} \mathrm{X}$-rays is about $20 \%$ lower while the response to $20 \mathrm{kVp} \mathrm{X}$-rays has fallen by about $50 \%$.

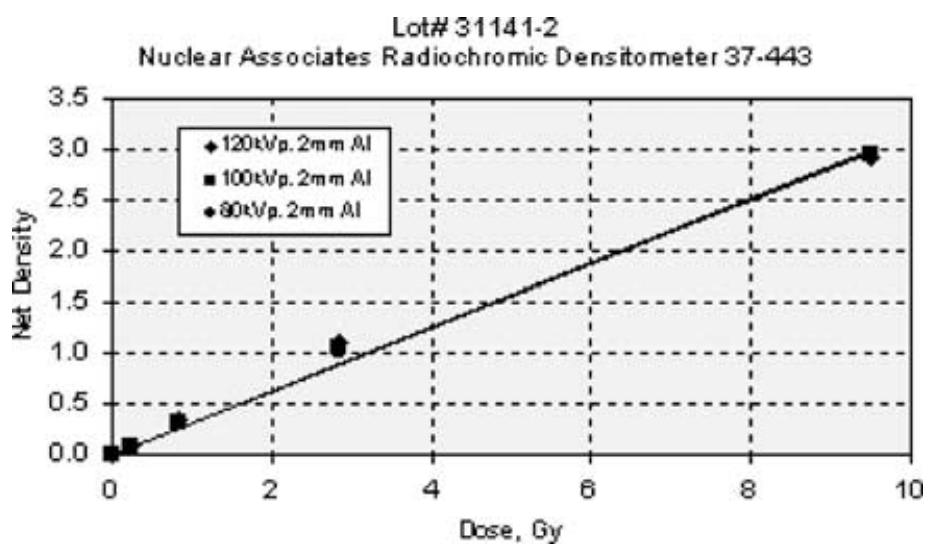

Fig. 41. Energy dependence of the GAFCHROMIC ${ }^{\circledR}$ XR type R radiochromic film in the range of $80-120 \mathrm{kVp}$ (results courtesy of David Lewis, ISP Technology). 


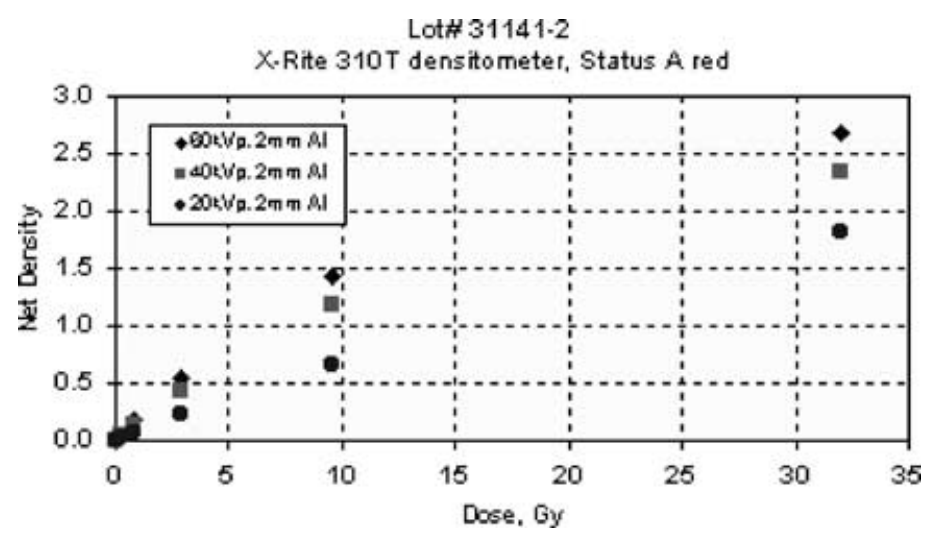

Fig. 42. Energy dependence of the GAFCHROMIC ${ }^{\circledR}$ XR type R radiochromic film in the range of $60-20 \mathrm{kVp}$ (results courtesy of David Lewis, ISP Technology).

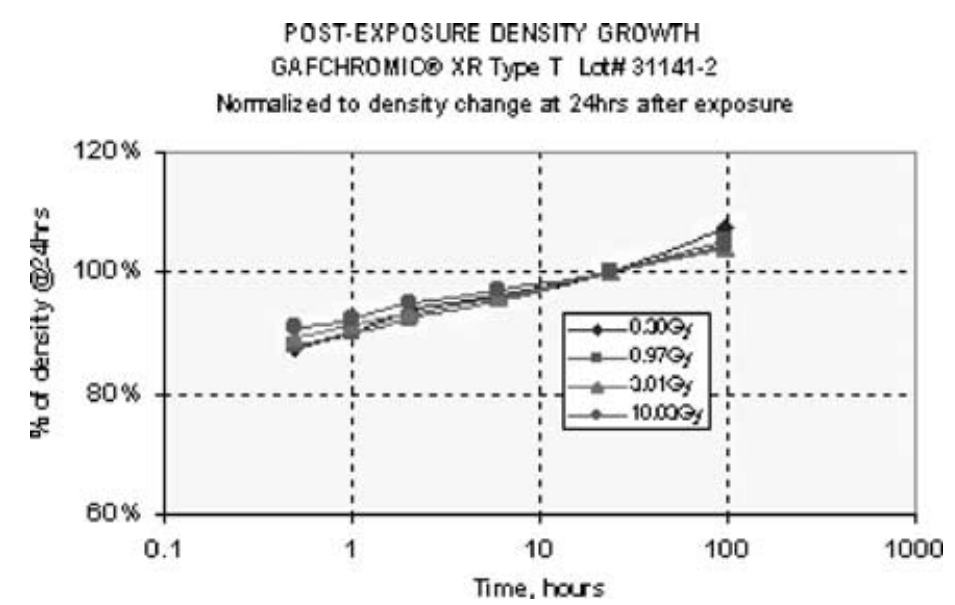

Fig. 43. Normalised post-irradiation optical density growth for the GAFCHROMIC ${ }^{\circledR}$ XR type T radiochromic film (results courtesy of David Lewis, ISP Technology).

The GAFCHROMIC ${ }^{\circledR}$ XR type $T$ radiochromic dosimetry film exhibits no dose rate or fractionation effects.

Fig. 43 shows the normalised post-irradiation growth response of the GAFCHROMIC ${ }^{\mathbb{R}}$ XR type $\mathrm{T}$ radiochromic film. This reveals that the post-exposure density growth, relative to the density at $24 \mathrm{~h}$, is essentially independent of the exposure dose, and is proportional to the logarithm of the time. The density changes by about $9 \%$ between $1 \mathrm{~h}$ after exposure and $24 \mathrm{~h}$ after exposure, but the rate diminishes and the density changes by only about $4 \%$ over the next $72 \mathrm{~h}$.

Film non-uniformity is $<3 \%$ as quoted by the manufacturer.

\section{Medical applications}

\subsection{Medical skin dosimetry}

When cancer patients are treated with radiation therapy beams, various skin reactions have been noticed. Early stage effects include erythema and in some cases desquamation [144]. Occasionally, 
late effects such as Telangiectasis may occur. Doses delivered in the first millimeter of skin are often dominated by electron contamination and can vary quite considerably within the first few millimetres of depth due to the build up characteristics of X-ray beams. These changes can be attributed to variations in electron contamination caused by parameters such as the field size, the use of beam modifying devices and factors involved with in-phantom scatter such as exit dose and beam angle of incidence [145]. This is one of the prime reasons that measurements are necessary to estimate the build up dose effects because this situation is not easily modelled by Monte Carlo-type calculations. Normally, a patient is treated either supine or prone. If anterior and posterior beams are used, usually one of the beams must traverse through the linear accelerator treatment couch. The linear accelerator couch is normally made from carbon fibre in a tennis string formation with a mylar covering for comfort. The introduction of this material into the beam path will increase the dose delivered to the patient's skin during treatment. Radiochromic film has proved quite successful in measuring these clinical changes to skin dose on a patient-specific basis. Fig. 44 shows a profile of skin dose measured with MD-55-2 radiochromic films when treating through a carbon fibre support couch as is used clinically with linear accelerators. Results are at depths of $0.17 \mathrm{~mm}$ (effective depth of measurement for this film) and at $1 \mathrm{~mm}$ depth (dermal layer of skin).

A silicon-based burn's dressing can be used as a protective layer for skin during radiotherapy. This dressing may reduce skin damage caused by excess friction or rubbing of the irradiated area on clothes or other materials during treatment [146]. The use of such a dressing, which is normally left in place for an extended period of time, introduces scatter material in the beam path during irradiation, similar to bolus thus effectively increasing skin dose as a consequence. Radiochromic film has proved useful in the measurement of these increases on a small scale using two-dimensional dosimetry not able to be performed with conventional dosimeters. Fig. 45 shows a dose map created with radiochromic MD-55-2 films, which has measured dose under a MEPITEL silicon.

Assessment of the skin dose delivered to patients from radiotherapy X-ray beams should be performed both inside and outside the prescribed treatment fields. A multi-layer GAFCHROMIC ${ }^{\mathbb{R}}$ film detector with a high sensitivity for detection of radiation can be used to measure the skin dose in a two-dimensional map over the skin surface. This is an advantage over other detectors which only provide point dose estimates. Results from a study by Cheung et al. [147] using radiochromic film

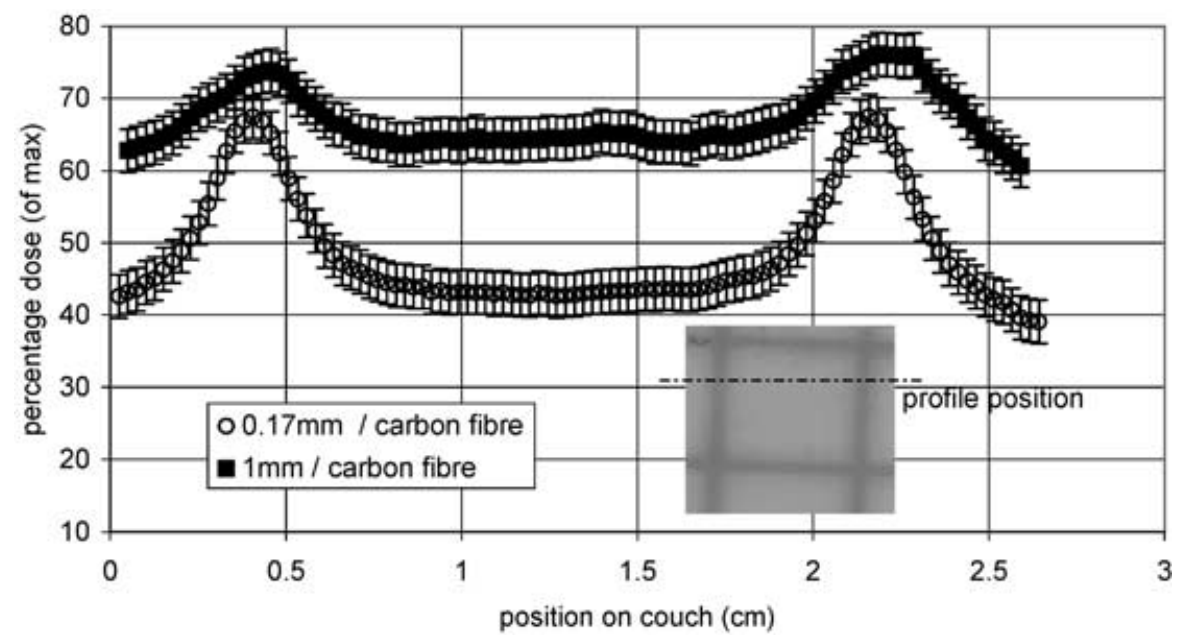

Fig. 44. Skin dose delivered to a patient through a Varian carbon fibre treatment couch as measured by a radiochromic film. Doses at approximately the basal layer and dermal layer of the skin are seen with larger doses deposited at the carbon fibre inserts. 


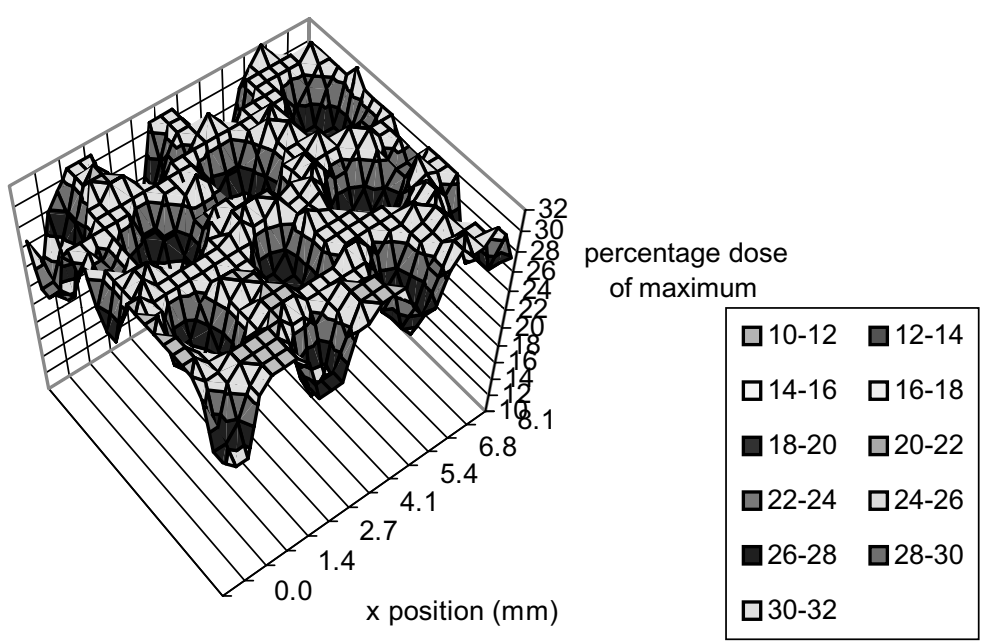

Fig. 45. Skin dose delivered under a MEPITEL silicon-based burn's dressing used in radiotherapy for reduction of skin damage due to rubbing and friction of the treated site on clothes or other material. A two-dimensional map of dose is created by radiochromic film dosimeters.

detectors showed that the main contributor to the total skin dose within the treatment field was delivered by the exit dose. However, outside the field boundary, dose was still delivered by entry beams. Fig. 46 shows a break down of skin dose contributions from the entry beam and the exit beam as measured on the same patient on the medial side over a five-fraction period but quoted as dose per fraction. As can be seen, the majority of the skin dose delivered within the treatment field is due to the exit dose from the opposing beam. However, the dose outside the field is mainly due to electron contamination from the entry field. The dose deposited from the entry beam does not have a large variation in the 'penumbral' region and this is mainly caused by the fact that electron contamination is spread out and extends beyond the geometric field edge and the influence of phantom scatter is small at this depth. Results within the field for medial and lateral beams (parallel opposing beam

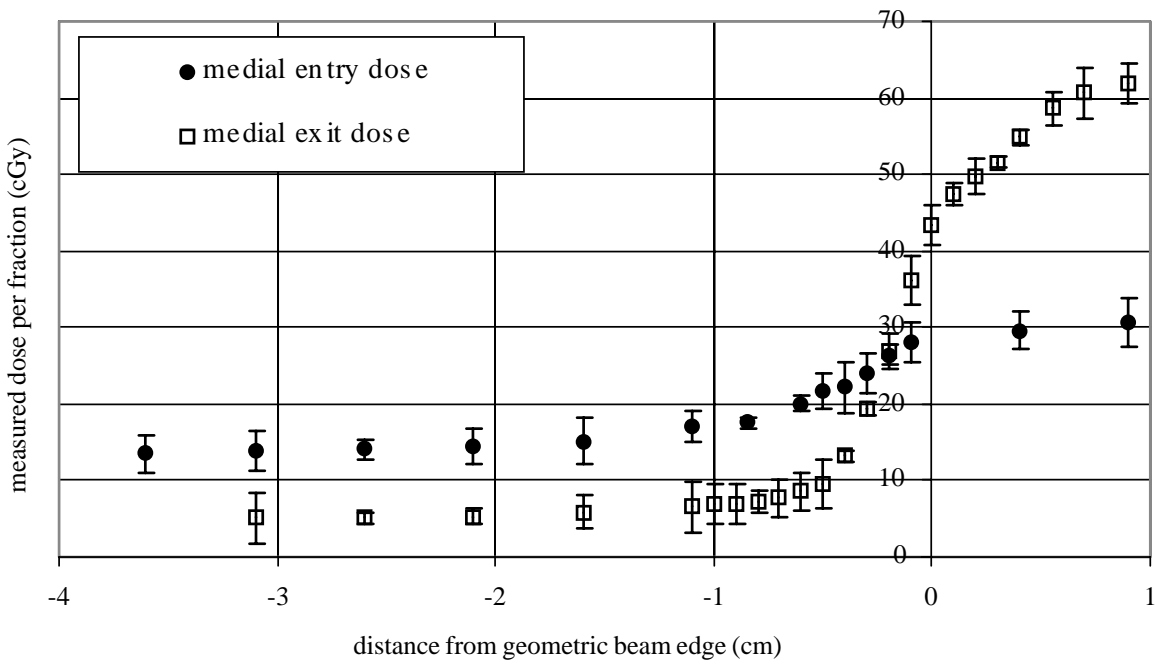

Fig. 46. Skin dose delivered in vivo to a patient undergoing breast cancer radiotherapy. The dose measured are for exiting photon beams and entry photon beams highlighting the variations in skin dose delivered by these two types of treatment beams as measured by the radiochromic film. 
configuration to best deliver dose to a breast) do show a difference in measured dose with skin dose delivered on the medial side producing a larger size than at the lateral beam edge. Again, this is directly attributed to the variations in the dose delivered for varying angle of incidences. Due to the design of the treatment procedure, the medial beam angle of incidence would be larger than that for the lateral beam, especially near the inferior beam edge where measurements were taken. The increased dose delivered highlights the effect from the angle of incidence. The multi-layer film produces adequate assessments of the dose and can assess the dose at multiple points both within and outside of the field. These results have shown that a significant amount of dose is deposited in the skin region, outside the target volume/treatment field.

\subsection{Medical applications summary}

The use of radiochromic film in medical applications is extensive ranging from high-dose $\gamma$-ray exposure as in brachytherapy through to low-dose clinical assessment in vivo such as conventional radiotherapy of breast patients. Although this review concentrates on the theory of medical radiation dosimetry and physical characteristics of the radiochromic film, a synopsis (with brief clinical reference list) of some medical applications is included to provide an insight into the medical radiochromic dosimetry realm and its usefulness.

Proton dosimetry [148-154] has been shown to be possible using various radiochromic film products. There seems to be an approximate linear response of $4.4 \%$ for 1 standard deviation within a 10-100 Gy dose region for high-energy proton beams, except near the Bragg peak location where it exhibits a 5-10\% dose suppression. The radiochromic film has been useful for quantitative evaluation as well as spatial resolution measurements to detect misalignments greater than $2 \mathrm{~mm}$ in the treatment technique.

Surface and skin dosimetry [155-163] has been studied with the use of radiochromic film products due to their low-energy dependence, their relatively small effective thickness and the ability to produce a two-dimensional dose map which is not currently available by other skin dosimeters in radiotherapy. Applications have included in vivo dosimetry as well as phantom studies for dose assessment at the surface, basal and dermal cell layers as well as subcutaneous tissue layers.

Brachytherapy [164-176] is the treatment of cancer with the use of radioactive sources placed within the patient's tissue. Steep dose gradients around brachytherapy sources mean that the radiochromic film, with its low sensitivity and high spatial resolution, has an advantage over other detectors and can be used for dosimetry near these high activity sources.

Radiosurgery [177-188]. For radiation beams with radii less than $1 \mathrm{~cm}$ used in stereotactic radiosurgery, there is a reduction in dose on the central axis due to electron disequilibrium, leading to measurement artefacts in large detectors. Radiochromic films have the resolution necessary to measure the absorbed dose for these narrow beams and these measurements allow the calculation of scatter factors for phantom-scattered photons. Calibrated radiochromic films can be used to measure the dose characteristics of these small fields and can be used for acceptance testing and quality control.

Radiation dosimetry near interfaces [189-191] of high-density change in humans can prove quite difficult due to electron disequilibrium in these regions. These can occur near bone-tissue interfaces, lung-tissue and internal cavities. Also, dental work and prosthesis made from highdensity materials can severely affect the dose delivered to the immediate area. The radiochromic film has been ideally suited to measurements in these regions.

Penumbral measurements [192-195] where the dose is delivered in the edge of the radiation beam is also usefully measured by radiochromic films. The penumbra region of dose delivery 
produces a high-dose gradient and changed spectral qualities compared to the central axis of a medical radiation beam. This has been useful for conventional radiotherapy collimated beams as well as newer multi-leaf collimated beams.

Intensity modulated radiation therapy (IMRT) [196-198] dose assessment is achievable in small segmented areas without the effects of energy response using radiochromic films. Conventional radiographic film detection and accuracy suffer from the energy dependence of the silver halide films in areas of low-energy photons making radiochromic films more advantageous for dosimetry in these areas.

\section{Conclusion}

The radiochromic film has become an essential part of radiation dosimetry. Properties such as its relatively energy-independent response to radiation and high spatial resolution make it ideal as a medical radiation dosimeter. Other advantages of the radiochromic film include its high precision, accuracy, large measurement range, dose-rate independence, ease of handling and no processing requirement. Many applications have been found for radiochromic films in medical radiation detection, and future expansion are expected as higher sensitivity films are created.

\section{Acknowledgements}

The authors wish to thank Mr. Dean Inwood for contributions to the manuscript preparation and for some research on polarization effects of radiochromic films. The authors also wish to thank Prof. David Lewis of ISP Technology, Prof. Scot Larson of FWT Inc. and Prof Gary Pageau of GEX Corporation for information regarding the radiochromic film products of their companies. This work has been fully supported by a grant from the Research Grants Council of Hong Kong Special Administrative Region, P.R. China (Project No. CityU 1012/01P).

\section{References}

[1] K. Siegbahn, Alpha, Beta and Gamma Ray Spectroscopy, North-Holland, Amsterdam, The Netherlands, 1965.

[2] J. McGervey, Introduction to Modern Physics, second ed., Academic Press, New York, 1983.

[3] O. Klein, Y. Nishina, Uber die streuung von Strahlung durch freie Electronen nach der neuen relativistischen Quanten dynamic von Dirac (On the scattering of radiation by free electrons based on the relativistic quantum dynamics of Dirac, Trans.), Zs. Phys. 52 (1929) 853.

[4] H.E. Johns, J.R. Cunningham, The Physics of Radiology, fourth ed., Thomas, Sprinfield, IL, 1983

[5] S.C. Klevenhagen, Physics of Electron Beam Therapy, first ed., Adam Hilger, Bristol, UK, 1985.

[6] M.J. Berger, S.M. Seltzer, Stopping Power Tables, NASA SP3036, Washington, DC, 1966.

[7] L. Eyges, Phys. Rev. 74 (1948) 1534.

[8] K.R. Hogstrom, Med. Phys. 12 (1985) 554.

[9] D.J. Perry, J.G. Holt, Med. Phys. 7 (1980) 207.

[10] D.J. Jette, Am. Assoc. Med. Dosim. 9 (1984) 6.

[11] D.J. Jette, Am. Assoc. Med. Dosim. 9 (1984) 12.

[12] A. Brahme, I. Lax, P. Andreo, Acta Radiat. Oncol. 20 (1981) 147.

[13] I. Lax, Phys. Med. Biol. 31 (1986) 879.

[14] W.R. Nelson, H. Hirayama, D.W.O. Rogers, The EGS4 Code System, Stanford Linear Accelerator Centre Publication, Stanford, 1985, p. 265.

[15] International Commission on Radiological Units, Radiation Quantities and Units, ICRU Report 19, 1971.

[16] International Commission on Radiological Units, Determination of Absorbed Dose in a Patient Irradiated with Beams of X or Gamma Rays in Radiation Procedures, ICRU Report 24, 1976. 
[17] F.H. Attix, Health Phys. 36 (1979) 347.

[18] J.R. Greening, Fundamentals of Radiation Dosimetry, first ed., Adam Hilger, Bristol, England, 1981.

[19] P.E. Metcalfe, P.W. Hoban, D.C. Murray, W.H. Round, Aust. Phys. Eng. Sci. Med. 12 (1989) 138.

[20] S.G. Ju, Y.C. Ahn, S.J. Huh, I.J. Yeo, Med. Phys. 29 (2002) 351.

[21] N. Suchowerska, P. Hoban, M. Butson, A. Davison, P. Metcalfe, Phys. Med. Biol. 46 (2001) 1391.

[22] J.L. Robar, B.G. Clark, Med. Phys. 26 (1999) 2144.

[23] C. Danciu, B.S. Proimos, J.C. Rosenwald, B.J. Mijnheer, Med. Phys. 28 (2001) 966.

[24] W. McLaughlin, in: N.W. Holm, R.J. Berry (Eds.), Film, Dyes and Photographic Systems, Manual on Radiation Dosimetry, Marcel Dekker, New York, 1970.

[25] J. Kosar, Light Sensitive Systems, Wiley, New York, 1965.

[26] G.H. Brown (Ed.), Photochromism, Wiley/Interscience, New York, 1971.

[27] P. Metcalfe, T. Kron, P. Hoban, The Physics of Radiotherapy X-rays from Linear Accelerators, Medical Physics Publishing, Madison, WI, 1997.

[28] A. Niroomand-Rad, C.R. Blackwell, B.M. Coursey, K.P. Gall, J.M. Galvin, W.L. McLaughlin, A.S. Meigooni, R. Nath, J.E. Rodgers, C.G. Soares, Med. Phys. 25 (1998) 2093.

[29] G. Dorian, A. Wiebe, Photochromism, Focal Press, London, 1970.

[30] J. Margerum, L. Miller, Photochromic Processes by Todtomerism, Photochromism Techniques in Chemistry, Wiley, New York, 1971.

[31] J. Rust, J. Margerum, L. Miller, Light scattering imaging by photopolymerization, in: R.D. Murray (Ed.), Proceedings of the Seminars on Novel Imaging Systems, Washington, DC, 1969.

[32] J. Arney, J. Imaging Sci. 33 (1989) 1.

[33] W. Lawton, Recent advances in organic-based imaging systems, in: R.D. Murray (Ed.), Proceedings of the Seminars on Novel Imaging Systems, Washington, DC, 1969.

[34] W. McLaughlin, in: N.W. Holm, R.J. Berry (Eds.), Film, Radiochromic Dye-Cyanide Dosimeters, Manual on Radiation Dosimetry, Marcel Dekker, New York, 1970.

[35] W. Mclaughlin, L. Chalkley, Phon. Sci. Eng. 9 (1965) 159.

[36] D. Lewis, A processless electron recording medium, in: Proceedings of the SPSE'86 Symposium on Electronic Imaging, Arlington, VA, 1986.

[37] M. Saylor, T. Tamargo, W. Mclaughlin, H. Khan, D. Lewis, R. Schenfele, Radiat. Phys. Chem. 31 (1988) 529.

[38] W. Mclaughlin, Y. Chen, C. Soares, G. Miller, G. Van Dyke, D. Lewis, Nucl. Instrum. Methods Phys. Res. A 302 (1991) 165.

[39] W. McLaughlin, M. Al-Sheikhly, D. Lewis, A. Kovacs, L. Wojnarovits, A radiochromic solid state polymerisation reaction, in: R.L. Clough (Ed.), Proceedings of the Symposium on Radiation Effects on Polymers, Washington, DC, 1994, pp. 920-921 (unpublished, as cited by Niroomand-Rad, et al., Med. Phys. TG-55 (1998)).

[40] W. McLaughlin, M. Al-Sheikhly, D. Lewis, A. Kovacs, L. Wojnarovits, in: R. Clough (Ed.), Radiochromic Solid State Polymerisation Reaction, Irradiation of Polymers, American Chemical Society, Washington, DC, 1996, pp. 152-166.

[41] W. McLaughlin, J. Puhl, M. Al-Shiekhly, C. Christou, A. Miller, A. Kovacs, L. Wojnarovits, D. Lewis, Radiat. Prot. Dosim. 66 (1996) 263

[42] N. Klassen, L. van der Zwan, J. Cygler, Med. Phys. 24 (1997) 1924.

[43] J. Huang, L. Barbera, M. Brouwers, G. Browman, W.J. Mackillop, J. Clin. Oncol. 21 (2003) 555.

[44] L. Jones, P. Hoban, P. Metcalfe, Aust. Phys. Eng. Sci. Med. 24 (2001) 132.

[45] N.S. Russell, H. Bartelink, Radiotherapy: the last 25 years, Cancer Treat. Rev. 25 (1999) 365.

[46] D. Lewis, Product information data for Gafchromic MD-55-2 radiochromic film, 2002, http://www.isp.copr.com.

[47] E.R. Giles, P.H. Murphy, Health Phys. 82 (2002) 875.

[48] T. Wiezorek, M. Schwedas, M. Scheithauer, H. Salz, M. Bellemann, T.G. Wendt, Strahlenther. Onkol. 178 (2002) 732.

[49] R.D.H. Chu, G. Van Dyk, D.F. Lewis, K.P.J. O’Hara, B.W. Buckland, F. Dinelle, Radiat. Phys. Chem. 35 (1990) 767-773.

[50] Z. Li, D. Wen, D. Chen, S. Peng, L. Zhang, K. Shi, Radiat. Phys. Chem. 57 (2000) 103.

[51] J.W. Hansen, M. Wille, K.J. Olsen, Radiat. Phys. Chem. 23 (1984) 455.

[52] W. McLaughlin, et al., Irradiation of Polymers Fundamentals and Technological Applications, ACS Symposium Series, American Chemical Society, 1996 (Chapter 11).

[53] W. McLaughlin, in: E. Godghran (Ed.), Solid Phase Chemical Dosimeters Sterilization by Ionising Radiation, Multiscience, Montreal, vol. 1, 1974, pp. 219-324.

[54] V. Danchenko, G. Griffen, S. Brashears, IEEE Trans. Nucl. Sci. NS-28 (1981) 4156.

[55] L.E. Reinstein, G.R. Gluckman, A.G. Meek, Phys. Med. Biol. 43 (1998) 2703.

[56] L.E. Reinstein, G.R. Gluckman, H.I. Amols, Med. Phys. 24 (1997) 1935.

[57] Y. Zhu, A.S. Kirov, V. Mishra, A.S. Meigooni, J.F. Williamson, Med. Phys. 24 (1997) 223.

[58] A.S. Meigooni, M.F. Sanders, G.S. Ibbott, S.R. Szeglin, Med. Phys. 23 (1996) 1883.

[59] S. Nablo, E. Tripp, Radiat. Phys. Chem. 9 (1977) 325. 
[60] R. Uribe, M. Barcelo, W. McLaughlin, A. Beunfil, J. Rios, Radiat. Phys. Chem. 35 (1990) 724.

[61] M. Butson, P.K.N. Yu, Med. Phys. 25 (1998) 793.

[62] J.F. Dempsey, D.A. Low, S. Mutic, J. Markman, A.S. Kirov, G.H. Nussbaum, J.F. Williamson, Med. Phys. 27 (2000) 2462.

[63] B. Mersseman, C. De Wagter, Phys. Med. Biol. 43 (1998) 1803.

[64] X. Wang, S. Spirou, T. LoSasso, J. Stein, C.S. Chui, B. Mohan, Med. Phys. 23 (1996) 317.

[65] A.J. Olch, Med. Phys. 29 (2002) 2159.

[66] M.J. Butson, R. Elferink, T. Cheung, P.K.N. Yu, M. Stokes, K.Y. Quach, P. Metcalfe, Phys. Med. Biol. 45 (2000) N143.

[67] M.J. Butson, T. Cheung, P.K.N. Yu, Radiat. Meas. 35 (2002) 99.

[68] G.R. Gluckman, J. Foraci, A.G. Meek, L.E. Reinstein, Int. J. Radiat. Oncol. Biol. Phys. 51 (Suppl. 1) (2001) 171.

[69] T. Kron, L. DeWerd, P. Mobit, J. Muniz, A. Pradhan, M. Toivonen, M. Waligorski, Phys. Med. Biol. 44 (1999) L15.

[70] T. Kron, M. Butson, F. Hunt, J. Denham, Radiother. Oncol. 41 (1996) 119.

[71] P.M. Ostwald, T. Kron, C.S. Hamilton, J.W. Denham, Int. J. Radiat. Oncol. Biol. Phys. 33 (1995) 943.

[72] T. Kron, Aust. Phys. Eng. Sci. Med. 18 (1995) 1.

[73] N.D. Richmond, R.H. Kermode, Med. Dosim. 27 (2002) 25.

[74] M. Essers, L. van Battum, B.J. Heijmen, Radiother. Oncol. 61 (2001) 185.

[75] R.J. Watts, Med. Phys. 25 (1998) 247.

[76] T. Ackerly, S. Todd, I. Williams, M. Geso, J. Cramb, Aust. Phys. Eng. Sci. Med. 20 (1997) 71.

[77] M.J. Butson, J.N. Mathur, P.E. Metcalfe, Phys. Med. Biol. 41 (1996) 1073.

[78] M.J. Butson, P.K.N. Yu, P.E. Metcalfe, Phys. Med. Biol. 43 (1998) 2407.

[79] P.O. Kellermann, A. Ertl, E. Gornik, Phys. Med. Biol. 43 (1998) 2251.

[80] M.J. Butson, T. Cheung, P.K.N. Yu, D. Abbati, G.E. Greenoak, Phys. Med. Biol. 45 (2000) 1863.

[81] D.O. Odero, G.R. Gluckman, K. Welsh, R.A. Wlodarczyk, L.E. Reinstein, Med. Phys. 28 (2001) 1446.

[82] M.G. Carolan, M.J. Butson, K. Herrmann, J.N. Mathur, P.E. Metcalfe, Aust. Phys. Eng. Sci. Med. 20 (1997) 183.

[83] P.R. Sullivan, B.F. Hasson, C.H. Grossman, L.D. Simpson, Med. Phys. 27 (2000) 245.

[84] G.H. Chan, W.V. Prestwich, Phys. Med. Biol. 45 (2000) 1851.

[85] A. Niroomand-Rad, Med. Phys. 24 (1997) 1317.

[86] P.R. Sullivan, B.F. Hasson, C.H. Grossman, L.D. Simpson, Med. Phys. 27 (2000) 245-251.

[87] D.S. Berger, Photochem. Photobiol. 24 (1976) 587.

[88] S. Wengraitis, D. Benedetta, D.H. Sliney, Photochem. Photobiol. 68 (1998) 179.

[89] A. Davis, G.H.W. Deane, B.L. Diffey, Nature 261 (1976) 169.

[90] B. Diffey, in: B. Diffey (Ed.), Ultraviolet Radiation Dosimetry with Polysulphone Film Radiation Measurements in Photobiology, Academic Press, New York, 1989, pp. 136-159.

[91] C. Wong, R. Fleming, S. Carter, Photochem. Photobiol. 50 (1989) 611.

[92] A.A. Abdel-Fattah, A.H. El-sayed, H. Ezz El-Din, J. Photochem. Photobiol. A: Chem. 137 (2000) 37.

[93] S. Ebraheem, A.A. Abdel-Fattah, F.I. Said, Z.I. Ali, Radiat. Phys. Chem. 57 (2000) 195.

[94] F. Abdel Rehim, A. Ali Basfar, A. Abdel-Fattah, J. Photochem. Photobiol. A: Chem. 101 (1996) 63.

[95] N.V. Klassen, L. van der Zwan, J. Cygler, Med. Phys. 24 (1997) 1924.

[96] I. Dean, personal communication, 2003.

[97] G. Takacs, personal communication, 2003.

[98] L. Reinstein, Med. Phys. 22 (1995) 1011.

[99] A. Fidanzio, L. Azario, C. De Angelis, M. Pacilio, S. Onori, A. Kacperek, A. Piermattei, Med. Phys. 29 (2002) 669.

[100] A. Piermattei, R. Miceli, L. Azario, A. Fidanzio, S. delle Canne, C. De Angelis, S. Onori, M. Pacilio, E. Petetti, L. Raffaele, M.G. Sabini, Med. Phys. 27 (2000) 1655.

[101] P.J. Muench, A.S. Meigooni, R. Nath, W.L. McLaughlin, Med. Phys. 18 (1991) 769.

[102] M.J. Butson, T. Cheung, P.K.N. Yu, Phys. Med. Biol. 47 (2002) N285.

[103] J. Sayeg, C. Coffey, W. McLaughlin et al., The energy response of Gafchromic radiation detector, Med. Phys. 17 (1990) 521 (Abstract).

[104] M. Angelone, M. Chiti, A. Esposito, E. Fantuzzi, F. Monteventi, Radiat. Prot. Dosim. 100 (2002) 381.

[105] P. Olko, P. Bilski, J.L. Kim, Radiat. Prot. Dosim. 100 (2002) 119.

[106] T.C. Chu, S.Y. Lin, C.C. Hsu, J.P. Li, Appl. Radiat. Isot. 55 (2001) 679.

[107] T. Kron, L. Duggan, T. Smith, A. Rosenfeld, M. Butson, G. Kaplan, S. Howlett, K. Hyodo, Phys. Med. Biol. 43 (1998) 3235.

[108] S. Onori, C. De Angelis, P. Fattibene, M. Pacilio, E. Petetti, L. Azario, R. Miceli, A. Piermattei, L.B. Tonghi, G. Cuttone, S. Lo Nigro, Phys. Med. Biol. 45 (2000) 3045.

[109] S.N. Rustgi, Med. Phys. 22 (1995) 567.

[110] R.C. McCall, T.M. Jenkins, G.D. Oliver Jr., Med. Phys. 5 (1978) 37.

[111] L.J. van Battum, B.J. Heijmen, Int. J. Radiat. Oncol. Biol. Phys. 18 (1990) 69.

[112] J. Dutreix, A. Dutreix, Film dosimetry of high energy electrons, Ann. N. Y. Acad. Sci. 161 (1969) 33.

[113] J.I. Hale, A.T. Kerr, P.C. Shragge, Med. Dosim. 19 (1994) 43. 
[114] J. Sykes, H. James, P. Williams, Med. Phys. 26 (1999) 329.

[115] S.L. Dong, T.C. Chu, G.Y. Lan, T.H. Wu, Y.C. Lin, J.S. Lee, Appl. Radiat. Isot. 57 (2002) 883.

[116] C.R. Edwards, S. Green, J.E. Palethorpe, P.J. Mountford, Phys. Med. Biol. 42 (1997) 2383.

[117] D.J. Gladstone, X.Q. Lu, J.L. Humm, H.F. Bowman, L.M. Chin, Med. Phys. 21 (1994) 1721.

[118] M.J. Butson, T. Cheung, P.K.N. Yu, Phys. Med. Biol. 46 (2001) N27.

[119] N. Suchowerska, P. Hoban, A. Davison, P. Metcalfe, Phys. Med. Biol. 44 (1999) 1755.

[120] J.F. Williamson, F.M. Khan, S.C. Sharma, Med. Phys. 8 (1981) 94.

[121] GEX Corporation, personal communication.

[122] A. Miller, W. Batsberg, W. Karman, Radiat. Phys. Chem. 31 (1988) 491-496.

[123] T.N. Teslow, J. Digit. Imaging 10 (3 (Suppl. 1)) (1997) 128.

[124] J.F. Dempsey, D.A. Low, A.S. Kirov, J.F. Williamson, Med. Phys. 26 (1999) 1721.

[125] M. Bazioglou, J. Kalef-Ezra, Appl. Radiat. Isot. 55 (2001) 339.

[126] L.E. Reinstein, G.R. Gluckman, Med. Phys. 24 (1997) 1531.

[127] T. Cheung, M.J. Butson, P.K.N. Yu, Phys. Med. Biol. 47 (2002) N259.

[128] M.J. Butson, T. Cheung, P.K.N. Yu, Radiother. Oncol. 61 (2001) 211.

[129] G.R. Gluckman, L.E. Reinstein, Med. Phys. 29 (2002) 1839.

[130] T. Cheung, M.J. Butson, P.K.N. Yu, Phys. Med. Biol. 46 (2001) N197.

[131] A. Ertl, P.O. Kellermann, M. Zehetmayer, A. Schoggl, P. Kindl, A.H. Maitz, Med. Phys. 26 (1999) 834.

[132] M.A. Stevens, J.R. Turner, R.P. Hugtenburg, P.H. Butler, Phys. Med. Biol. 41 (1996) 2357.

[133] S.K. Chen, L. Hollender, J. Dent. 23 (1995) 205.

[134] A.S. Aydarous, P.J. Darley, M.W. Charles, Phys. Med. Biol. 46 (2001) 1379.

[135] A. Burch, D.A. Goodman, S.J. Hill, J. Law, A. Robinson, A.C. Underwood, Br. J. Radiol. 66 (1993) 552.

[136] H. Alva, H. Mercado-Uribe, M. Rodriguez-Villafuerte, M.E. Brandan, Phys. Med. Biol. 47 (2002) 2925.

[137] D.O. Odero, G.R. Gluckman, K. Welsh, R.A. Wlodarczyk, L.E. Reinstein, Med. Phys. 28 (2001) 1446.

[138] Dick Smith Pty Ltd., Technical specifications product catalogue—diode spectral response (visible region), 1998, p. 386.

[139] ASTM Standard Practice E-1275 (ISO 51275), Standard Practice for Use of a Radiochromic Film Dosimetry System.

[140] A. Adbel-Fattah, A. Miller, Radiat. Phys. Chem. 47 (1996) 611.

[141] ASTM Standard guide E-1261 (ISO 51261), Standard Guide for Selection and Calibration of Dosimetry Systems for Radiation Processing, NPL Report, CIRM 29, Guidelines for the Calibration of Dosimeters Used for Radiation Processing.

[142] Far West Technology, personal communication, 2003.

[143] ISP Technology, personal communication, 2003.

[144] I. Turesson, Int. J. Radiat. Oncol. Biol. Phys. 19 (1990) 1569.

[145] M.J. Butson, M.D. Perez, J.N. Mathur, P.E. Metcalfe, Aust. Phys. Eng. Sci. Med. 19 (1996) 74.

[146] M.J. Butson, T. Cheung, P.K.N. Yu, P. Metcalfe, Phys. Med. Biol. 47 (2002) N145.

[147] T. Cheung, M.J. Butson, P.K.N. Yu, Phys. Med. Biol. 47 (2002) N31.

[148] S.O. Troja, E. Egger, P. Francescon, A.M. Gueli, A. Kacperek, M.R. Coco, Technol. Health Care 8 (2000) 155.

[149] A. Piermattei, R. Miceli, L. Azario, A. Fidanzio, S. delle Canne, C. De Angelis, S. Onori, M. Pacilio, E. Petetti, L. Raffaele, M.G. Sabini, Med. Phys. 27 (2000) 1655

[150] S.M. Vatnitsky, D.W. Miller, M.F. Moyers, R.P. Levy, R.W. Schulte, J.D. Slater, J.M. Slater, Phys. Med. Biol. 44 (1999) 2789.

[151] S.M. Vatnitsky, R.W. Schulte, R. Galindo, H.J. Meinass, D.W. Miller, Phys. Med. Biol. 42 (1997) 1887.

[152] S.M. Vatnitsky, Appl. Radiat. Isot. 48 (1997) 643.

[153] D. Nichiporov, V. Kostjuchenko, J.M. Puhl, D.L. Bensen, M.F. Desrosiers, C.E. Dick, W.L. McLaughlin, T. Kojima, B.M. Coursey, S. Zink, Appl. Radiat. Isot. 46 (1995) 1355.

[154] I. Daftari, C. Castenadas, P.L. Petti, R.P. Singh, L.J. Verhey, Phys. Med. Biol. 44 (1999) 2735.

[155] T. Cheung, M.J. Butson, P.K.N. Yu, Phys. Med. Biol. 46 (2001) N235.

[156] M.J. Butson, T. Cheung, P.K.N. Yu, P.E. Metcalfe, Int. J. Radiat. Oncol. Biol. Phys. 46 (2000) 1071.

[157] C. Thilmann, I.A. Adamietz, S. Mose, F. Saran, U. Ramm, H.D. Bottcher, Radiother. Oncol. 40 (1996) 181.

[158] A.R. Hounsell, J.M. Wilkinson, Phys. Med. Biol. 44 (1999) 43.

[159] M.J. Butson, J.N. Mathur, P.E. Metcalfe, Phys. Med. Biol. 41 (1996) 1073.

[160] M.J. Butson, P.K.N. Yu, P.E. Metcalfe, Phys. Med. Biol. 43 (1998) 2647.

[161] K.Y. Quach, J. Morales, M.J. Butson, A.B. Rosenfeld, P.E. Metcalfe, Med. Phys. 27 (2000) 1676.

[162] E.R. Giles, P.H. Murphy, Health Phys. 82 (2002) 875.

[163] M. Amano, H. Nishitani, S. Kohno, M. Yasutomo, H. Miyoshi, H. Yagi, Nippon Hoshasen Gijutsu Gakkai Zasshi 59 (2003) 121.

[164] A. Piermattei, A. Fidanzio, L. Azario, A. Russo, F. Perrone, R. Capote, M.P. Toni, Phys. Med. Biol. 47 (2002) 4205.

[165] A. Martinez-Davalos, M. Rodriguez-Villafuerte, R. Diaz-Perches, S. Arzamendi-Perez, Radiat. Prot. Dosim. 101 (2002) 489. 
[166] M.W. Skwarchuk, T.G. Ochran, R. Komaki, J. Cundiff, E.L. Travis, Int. J. Radiat. Oncol. Biol. Phys. 34 (1996) 173.

[167] T.D. Bohm, D.W. Pearson, R.K. Das, Med. Phys. 28 (2001) 142.

[168] L. Duggan, M. Butson, S. Howlett, J. Denham, T. Kron, Aust. Phys. Eng. Sci. Med. 23 (2000) 15.

[169] D.M. Duggan, C.W. Coffey II, J.L. Lobdell, M.C. Schell, Med. Phys. 26 (1999) 2461.

[170] W. Schumer, W. Fernando, M. Carolan, T. Wong, S. Wallace, G. Quong, M. Geso, Med. Dosim. 24 (1999) 197.

[171] S. Pai, L.E. Reinstein, G. Gluckman, Z. Xu, T. Weiss, Med. Phys. 25 (1998) 1217.

[172] K.D. Steidley, Med. Dosim. 23 (1998) 37.

[173] P.J. Muench, A.S. Meigooni, R. Nath, W.L. McLaughlin, Med. Phys. 18 (1991) 769.

[174] C. Kirisits, D. Georg, P. Wexberg, B. Pokrajac, D. Glogar, R. Potter, Radiother. Oncol. 64 (2002) 309.

[175] G.H. Chan, W.V. Prestwich, Med. Phys. 29 (2002) 190.

[176] Y.C. Cheung, P.K.N. Yu, E.C.M. Young, C.L. Chan, M.F. Ng, F.N.F. Tang, T.P.Y. Wong, Appl. Radiat. Isot. 48 (1997) 985.

[177] M. Sanders, J. Sayeg, C. Coffey, P. Patel, J. Walsh, Stereotact. Funct. Neurosurg. 61 (Suppl. 1) (1993) 124.

[178] T.Y. Guan, P.R. Almond, H.C. Park, R.D. Lindberg, C.B. Shields, Med. Dosim. 18 (1993) 135.

[179] J.Y.C. Cheung, K.N. Yu, R.T. Ho, C.P. Yu, Med. Phys. 26 (1999) 1252.

[180] G.A. Horstmann, A.T. Van Eck, J. Neurosurg. 97 (2002) 450.

[181] A. Ertl, M. Zehetmayer, A. Schoggl, K. Kitz, R. Koschuch, H. Stadtmann, P. Kindl, Phys. Med. Biol. 43 (1998) 1567.

[182] J.Y.C. Cheung, K.N. Yu, R.T. Ho, C.P. Yu, Appl. Radiat. Isot. 53 (2000) 427.

[183] H.D. Kubo, F. Araki, Med. Phys. 29 (2002) 2497.

[184] W.L. McLaughlin, C.G. Soares, J.A. Sayeg, E.C. McCullough, R.W. Kline, A. Wu, A.H. Maitz, Med. Phys. 21 (1994) 379.

[185] R. Ramani, A.W. Lightstone, D.L. Mason, P.F. O’Brien, Med. Phys. 21 (1994) 389.

[186] A. Somigliana, G.M. Cattaneo, C. Fiorino, S. Borelli, A. del Vecchio, G. Zonca, E. Pignoli, G. Loi, R. Calandrino, R. Marchesini, Phys. Med. Biol. 44 (1999) 887.

[187] H. Shiomi, T. Inoue, S. Nakamura, T. Inoue, Radiat. Med. 18 (2000) 107.

[188] A. Mack, S.G. Scheib, J. Major, S. Gianolini, G. Pazmandi, H. Feist, H. Czempiel, H.J. Kreiner, Med. Phys. 29 (2002) 2080.

[189] A. Niroomand-Rad, K.W. Harter, S. Thobejane, K. Bertrand, Int. J. Radiat. Oncol. Biol. Phys. 29 (1994) 1139.

[190] A. Niroomand-Rad, R. Razavi, S. Thobejane, K.W. Harter, Int. J. Radiat. Oncol. Biol. Phys. 34 (1996) 475.

[191] A. Ralston, E. Estoesta, G. Stevens, A. Hong, Australas. Phys. Eng. Sci. Med. 24 (2001) 59.

[192] M.J. Butson, T. Cheung, P.K.N. Yu, Radiother. Oncol. 61 (2001) 211.

[193] T. Cheung, M.J. Butson, P.K.N. Yu, Phys. Med. Biol. 46 (2001) N235.

[194] P. Francescon, S. Cora, C. Cavedon, P. Scalchi, S. Reccanello, F. Colombo, Med. Phys. 25 (1998) 503.

[195] J.M. Galvin, K. Han, R. Cohen, Int. J. Radiat. Oncol. Biol. Phys. 40 (1998) 721.

[196] T. Wiezorek, M. Schwedas, M. Scheithauer, H. Salz, M. Bellemann, T.G. Wendt, Strahlenther. Onkol. 178 (2002) 732 .

[197] D.A. Low, S. Mutic, J.F. Dempsey, R.L. Gerber, W.R. Bosch, C.A. Perez, J.A. Purdy, Radiother. Oncol. 49 (1998) 305.

[198] P. Cadman, R. Bassalow, N.P. Sidhu, G. Ibbott, A. Nelson, Phys. Med. Biol. 47 (2002) 3001. 